\title{
Manifolds of mappings on Cartesian products
}

\author{
Helge Glöckner ${ }^{1}$. Alexander Schmeding ${ }^{2}$
}

Received: 8 September 2021 / Accepted: 24 November 2021 / Published online: 5 January 2022

(c) The Author(s) 2021

\begin{abstract}
Given smooth manifolds $M_{1}, \ldots, M_{n}$ (which may have a boundary or corners), a smooth manifold $N$ modeled on locally convex spaces and $\alpha \in\left(\mathbb{N}_{0} \cup\{\infty\}\right)^{n}$, we consider the set $C^{\alpha}\left(M_{1} \times \cdots \times M_{n}, N\right)$ of all mappings $f: M_{1} \times \cdots \times M_{n} \rightarrow N$ which are $C^{\alpha}$ in the sense of Alzaareer. Such mappings admit, simultaneously, continuous iterated directional derivatives of orders $\leq \alpha_{j}$ in the $j$ th variable for $j \in\{1, \ldots, n\}$, in local charts. We show that $C^{\alpha}\left(M_{1} \times \cdots \times M_{n}, N\right)$ admits a canonical smooth manifold structure whenever each $M_{j}$ is compact and $N$ admits a local addition. The case of non-compact domains is also considered.
\end{abstract}

Keywords Infinite-dimensional manifold · Infinite-dimensional Lie group · Compact-open topology $\cdot$ Exponential law $\cdot$ Evaluation map $\cdot$ Mapping group $\cdot$ Regularity $\cdot$ Box product . Non-compact manifold

Mathematics Subject Classification Primary: 58D15; secondary: 22E65 - 26E15 - 26E20 . $46 \mathrm{E} 40 \cdot 46 \mathrm{~T} 20$

\section{Introduction and statement of the results}

As known from classical work by Eells [9], the set $C^{\ell}(M, N)$ of all $C^{\ell}$-maps $f: M \rightarrow N$ can be given a smooth Banach manifold structure for each $\ell \in \mathbb{N}_{0}$, compact smooth manifold $M$ and $\sigma$-compact finite-dimensional smooth manifold $N$. More generally, $C^{\ell}(M, N)$ is a smooth manifold for each $\ell \in \mathbb{N}_{0} \cup\{\infty\}$, locally compact, paracompact smooth manifold $M$ with rough boundary in the sense of [15] (this includes finite-dimensional manifolds with boundary, and manifolds with corners as in [7,8,21]) and each smooth manifold $N$ modeled on locally convex spaces such that $N$ admits a local addition (a concept recalled in Defini-

H. Glöckner was supported by German Academic Exchange Service, DAAD Grant 57568548.

\footnotetext{
$\bowtie \quad$ Alexander Schmeding alexander.schmeding@nord.no

Helge Glöckner

glockner@math.uni-paderborn.de

1 Universität Paderborn, Warburger Str. 100, 33098 Paderborn, Germany

2 Nord universitet, Høgskoleveien 27, 7601 Levanger, Norway
} 
tion 5.6); see [4,14,16,21,22,25] for discussions in different levels of generality, and [20] for manifolds of smooth maps in the convenient setting of analysis. For compact $M$, the modeling space of $C^{\ell}(M, N)$ around $f \in C^{\ell}(M, N)$ is the locally convex space $\Gamma_{C^{\ell}}\left(f^{*}(T N)\right)$ of all $C^{\ell}$-sections in the pullback bundle $f^{*}(T N) \rightarrow M$, which can be identified with

$$
\Gamma_{f}:=\left\{\tau \in C^{\ell}(M, T N): \pi_{T N} \circ \tau=f\right\} ;
$$

if $M$ is not compact, the locally convex space of compactly supported $C^{\ell}$-sections of $f^{*}(T N)$ is used. Let $L$ be a smooth manifold modeled on locally convex spaces (possibly with rough boundary), and $k \in \mathbb{N}_{0} \cup\{\infty\}$. For compact $M$, it is known from [4, Proposition 1.23 and Definition 1.17] that a map

$$
g: L \rightarrow C^{\ell}(M, N)
$$

is $C^{k}$ if and only if the corresponding map of two variables,

$$
g^{\wedge}: L \times M \rightarrow N, \quad(x, y) \mapsto g(x)(y)
$$

is $C^{k, \ell}$ in the sense of [3], i.e., a continuous map which in local charts admits up to $\ell$ directional derivatives in the second variable, followed by up to $k$ directional derivatives in the first variable, with continuous dependence on point and directions (see 2.11 and 2.12 for details). We thus obtain a bijection

$$
\Phi: C^{k}\left(L, C^{\ell}(M, N)\right) \rightarrow C^{k, \ell}(L \times M, N), g \mapsto g^{\wedge} .
$$

As our first result, for compact $L$ we construct a smooth manifold structure on $C^{k, \ell}(L \times M, N)$ which turns $\Phi$ into a $C^{\infty}$-diffeomorphism. More generally, analogous to the $n=2$ case of $C^{k, \ell}$-maps, we consider $N$-valued $C^{\alpha}$-maps on an $n$-fold product $M_{1} \times \cdots \times M_{n}$ of smooth manifolds for any $n \in \mathbb{N}$ and $\alpha=\left(\alpha_{1}, \ldots, \alpha_{n}\right) \in\left(\mathbb{N}_{0} \cup\{\infty\}\right)^{n}$. With terminology explained presently, we get:

Theorem 1.1 Given $\alpha=\left(\alpha_{1}, \ldots, \alpha_{n}\right) \in\left(\mathbb{N}_{0} \cup\{\infty\}\right)^{n}$, let $M_{j}$ for $j \in\{1, \ldots, n\}$ be a compact smooth manifold with rough boundary. Let $N$ be a smooth manifold modeled on locally convex spaces such that $N$ can be covered by local additions. Then, the set $C^{\alpha}\left(M_{1} \times\right.$ $\left.\cdots \times M_{n}, N\right)$ admits a smooth manifold structure which is canonical. The following hold for this canonical manifold structure:

(a) $C^{\alpha}\left(M_{1} \times \cdots \times M_{n}, N\right)$ can be covered by local additions. If $N$ admits a local addition, then also $C^{\alpha}\left(M_{1} \times \cdots \times M_{n}, N\right)$ admits a local addition.

(b) Given $m \in \mathbb{N}$ and $\beta=\left(\beta_{1}, \ldots, \beta_{m}\right) \in\left(\mathbb{N}_{0} \cup\{\infty\}\right)^{m}$, let $L_{j}$ be a compact smooth manifold with rough boundary for $j \in\{1, \ldots, m\}$. Then, canonical smooth manifold structures turn the bijection

$$
\begin{aligned}
C^{\beta}\left(L_{1} \times \cdots \times L_{m},\right. & \left.C^{\alpha}\left(M_{1} \times \cdots \times M_{n}, N\right)\right) \\
& \rightarrow C^{\beta, \alpha}\left(L_{1} \times \cdots \times L_{m} \times M_{1} \times \cdots \times M_{n}, N\right)
\end{aligned}
$$

taking $g$ to $g^{\wedge}$ into a $C^{\infty}$-diffeomorphism.

The following terminology was used: We say that a smooth manifold $N$ can be covered by local additions if $N$ is the union of an upward directed family $\left(N_{j}\right)_{j \in J}$ of open submanifolds $N_{j}$ which admit a local addition. For instance, any (not necessarily paracompact) finite-dimensional smooth manifold has this property, e.g., the long line. We also used canonical manifold structures. 
Note that if a map $f: L_{1} \times \cdots \times L_{m} \times M_{1} \times \cdots \times M_{n} \rightarrow N$ on a product of smooth manifolds with rough boundary is $C^{\beta, \alpha}$ with $\alpha \in\left(\mathbb{N}_{0} \cup\{\infty\}\right)^{n}$ and $\beta \in\left(\mathbb{N}_{0} \cup\{\infty\}\right)^{m}$, then the map

$$
f^{\vee}(x):=f(x, \cdot): M_{1} \times \cdots \times M_{n} \rightarrow N
$$

is $C^{\alpha}$ for each $x \in L_{1} \times \cdots \times L_{m}$ (see [1, Lemma 3.3]).

Definition 1.2 Let $N$ be a smooth manifold modeled on a locally convex space, $M_{1}, \ldots, M_{n}$ be finite-dimensional smooth manifolds with rough boundary and $\alpha \in\left(\mathbb{N}_{0} \cup\{\infty\}\right)^{n}$. A smooth manifold structure on $C^{\alpha}\left(M_{1} \times \cdots \times M_{n}, N\right)$ is called pre-canonical if the following condition is satisfied for each $m \in \mathbb{N}$ and each $\beta \in\left(\mathbb{N}_{0} \cup\{\infty\}\right)^{m}$ : If $L_{j}$ for $j \in\{1, \ldots, m\}$ is a smooth manifold with rough boundary modeled on locally convex spaces, then a map

$$
g: L_{1} \times \cdots \times L_{m} \rightarrow C^{\alpha}\left(M_{1} \times \cdots \times M_{n}, N\right)
$$

is $C^{\beta}$ if and only if the map

$$
g^{\wedge}: L_{1} \times \cdots \times L_{m} \times M_{1} \times \cdots \times M_{n} \rightarrow N
$$

given by $g^{\wedge}\left(x_{1}, \ldots, x_{m}, y_{1}, \ldots, y_{n}\right):=g\left(x_{1}, \ldots, x_{m}\right)\left(y_{1}, \ldots, y_{n}\right)$ is $C^{\beta, \alpha}$. Thus,

$$
\begin{aligned}
C^{\alpha}\left(L_{1} \times \cdots \times L_{m},\right. & \left.C^{\beta}\left(M_{1} \times \cdots \times M_{n}, N\right)\right) \\
& \rightarrow C^{\beta, \alpha}\left(L_{1} \times \cdots \times L_{n} \times M_{1} \times \cdots \times M_{n}, N\right), \quad g \mapsto g^{\wedge}
\end{aligned}
$$

is a bijection. The manifold structure is called canonical if, moreover, its underlying topology is the compact-open $C^{\alpha}$-topology (as in Definition 3.4).

Canonical manifold structures are essentially unique whenever they exist, and so are precanonical ones (see Lemma 4.3 (b) for details).

We address two further topics for not necessarily compact domains:

(i) We formulate criteria ensuring that $C^{\alpha}\left(M_{1} \times \cdots \times M_{n}, G\right)$ admits a canonical smooth manifold structure (making the latter a Lie group), for a Lie group $G$ modeled on a locally convex space;

(ii) Manifold structures on $C^{\alpha}\left(M_{1} \times \cdots \times M_{n}, N\right)$ which are modeled on certain spaces of compactly supported $T N$-valued functions, in the spirit of [21].

To discuss (i), we use a generalization of the regularity concept introduced by John Milnor [22] (the case $r=\infty$ ). If $G$ is a Lie group modeled on a locally convex space, with neutral element $e$, we write $\lambda_{g}: G \rightarrow G, x \mapsto g x$ for left translation with $g \in G$ and consider the smooth left action

$$
G \times T G \rightarrow T G, \quad(g, v) \mapsto g \cdot v:=T \lambda_{g}(v)
$$

of $G$ on its tangent bundle. We write $\mathfrak{g}:=T_{e} G$ for the Lie algebra of $G$. Let $r \in \mathbb{N}_{0} \cup\{\infty\}$. The Lie group $G$ is called $C^{r}$-semiregular if, for each $C^{r}$-curve $\gamma:[0,1] \rightarrow \mathfrak{g}$, the initial value problem

$$
\dot{\eta}(t)=\eta(t) \cdot \gamma(t), \quad \eta(0)=e
$$

has a (necessarily unique) solution $\eta:[0,1] \rightarrow G$. Write $\operatorname{Evol}(\gamma):=\eta$. If, moreover, the map

$$
\text { Evol: } C^{r}([0,1], \mathfrak{g}) \rightarrow C^{r+1}([0,1], G)
$$

is smooth, then $G$ is called $C^{r}$-regular (cf. [12]). If $s \leq r$ and $G$ is $C^{s}$-regular, then $G$ is $C^{r}$-regular (see [12]). We show: 
Theorem 1.3 Let $G$ be a $C^{r}$-regular Lie group modeled on a locally convex space with $r \in \mathbb{N}_{0} \cup\{\infty\}$. For some $n \in \mathbb{N}$, let $M_{1}, \ldots, M_{n}$ be locally compact smooth manifolds with rough boundary and $\alpha \in\left(\mathbb{N}_{0} \cup\{\infty\}\right)^{n}$. For each $j \in\{1, \ldots, n\}$ such that $M_{j}$ is not compact, assume that $\alpha_{j} \geq r+1$ and $M_{j}$ is 1-dimensional with finitely many connected components. Then, we have:

(a) $C^{\alpha}\left(M_{1} \times \cdots \times M_{n}, G\right)$ admits a canonical smooth manifold structure;

(b) The canonical manifold structure from (a) makes $C^{\alpha}\left(M_{1} \times \cdots \times M_{n}, G\right) a C^{r}$-regular Lie group.

The Lie algebra of $C^{\alpha}\left(M_{1} \times \cdots \times M_{n}, G\right)$ can be identified with the topological Lie algebra $C^{\alpha}\left(M_{1} \times \cdots \times M_{n}, L(G)\right)$ in a standard way (Proposition 6.6). Of course, we are most interested in the case that the non-compact 1-dimensional factors are $\sigma$-compact and hence intervals, or finite disjoint unions of such. But we did not need to assume $\sigma$-compactness in the theorem, and thus $M_{j}$ with $\alpha_{j} \geq r+1$ might well be a long line, or a long ray.

Disregarding the issue of being canonical, the Lie group structure on $C^{\infty}\left(M_{1} \times \cdots \times M_{n}, G\right)=C^{\alpha}\left(M_{1} \times \cdots \times M_{n}, G\right)$ with $\alpha_{1}:=\cdots:=\alpha_{n}=\infty$ was first obtained in [24], for smooth manifolds $M_{j}$ without boundary which are compact or diffeomorphic to $\mathbb{R}$. The Lie group structure for $n=1$ was first obtained in [2] for domains diffeomorphic to intervals, together with a sketch for the case $n=2$ (assuming additional conditions, e.g., $\alpha_{1} \geq r+3$ and $\alpha_{2} \geq r+1$ if $M_{1}=M_{2}=\mathbb{R}$ ). Our approach differs: While the studies in [24] and [2] assume regularity of $G$ from the start to enforce exponential laws, and build it into a notion of Lie group structures on mapping groups that are "compatible with evaluations," we take canonical and pre-canonical manifold structures as the starting point (independent of regularity) and combine them with regularity or compatibility with evaluations (adapted to $C^{\alpha}$-maps in Definition 6.2) only when needed.

As to topic (b), our constructions show:

Theorem 1.4 Given $\alpha=\left(\alpha_{1}, \ldots, \alpha_{n}\right) \in\left(\mathbb{N}_{0} \cup\{\infty\}\right)^{n}$, let $M_{j}$ for $j \in\{1, \ldots, n\}$ be $a$ paracompact, locally compact smooth manifold with rough boundary; abbreviate $M:=$ $M_{1} \times \cdots \times M_{n}$. Let $N$ be a smooth manifold modeled on locally convex spaces such that $N$ admits a local addition. Let $\pi_{T N}: T N \rightarrow N$ be the canonical map. For $f \in C^{\alpha}(M, N)$ and a compact subset $K \subseteq M$, the set

$$
\Gamma_{f, K}:=\left\{\tau \in C^{\alpha}(M, T N): \pi_{T N} \circ \tau=f \& \tau(x)=0 \in T_{f(x)} N \text { for all } x \in M \backslash K\right\}
$$

is a vector subspace of $\prod_{x \in M} T_{f(x)} N$, and a locally convex space in the topology induced by $C^{\alpha}(M, T N)$. Give $\Gamma_{f}=\bigcup_{K} \Gamma_{f, K}$ the locally convex direct limit topology. Then, $C^{\alpha}(M, N)$ admits a unique smooth manifold structure modeled on the set $\mathcal{E}:=\left\{\Gamma_{f}: f \in C^{\alpha}(M, N)\right\}$ of locally convex spaces such that, for each $f \in C^{\alpha}(M, N)$ and local addition $\Sigma: T N \supseteq$ $U \rightarrow N$ of $N$, the map

$$
\Gamma_{f} \cap C^{\alpha}(M, U) \rightarrow C^{\alpha}(M, N), \tau \mapsto \Sigma \circ \tau
$$

is a $C^{\infty}$-diffeomorphism onto an open subset of $C^{\alpha}(M, N)$.

In the case that $n=1, k=\infty$ and $M:=M_{1}$ is a smooth manifold with corners, we recover the smooth manifold structure on $C^{\infty}(M, N)$ discussed by Michor [21].

Using manifold structures on infinite Cartesian products of manifolds making them "fine box products" (a concept recalled in Sect. 7), Theorem 1.4 turns into a corollary to Theorem 1.1. In the case $n=1$, for compact $M$ and $\ell \in \mathbb{N}_{0} \cup\{\infty\}$, canonical manifold structures on $C^{\ell}(M, N)$ as in Theorem 1.1 have already been considered in [4], in a weaker sense (fixing 
$m=1$ in Definition 1.2). Parts of our discussion adapt arguments from [4] to the more difficult case of $C^{\alpha}$-maps.

\section{Preliminaries and notation}

We write $\mathbb{N}:=\{1,2, \ldots\}$ and $\mathbb{N}_{0}:=\mathbb{N} \cup\{0\}$. If $\alpha, \beta \in\left(\mathbb{N}_{0} \cup\{\infty\}\right)^{n}$ with $n \in \mathbb{N}$, we write $\alpha \leq \beta$ if $\alpha_{j} \leq \beta_{j}$ for all $j \in\{1, \ldots, n\}$. We let $|\alpha|:=\alpha_{1}+\cdots+\alpha_{n} \in \mathbb{N}_{0} \cup\{\infty\}$. As usual, $\infty+k:=\infty$ for all $k \in \mathbb{N}_{0} \cup\{\infty\}$. For $j \in\{1, \ldots, n\}$, let $e_{j}:=(0, \ldots, 0,1,0, \ldots, 0) \in$ $\left(\mathbb{N}_{0}\right)^{n}$ with 1 in the $j$ th slot. We abbreviate "Hausdorff locally convex topological $\mathbb{R}$-vector space" as "locally convex space." We work in the setting of differential calculus going back to Andrée Bastiani [5] (see [10,15,16,21-23] for discussions in varying generality), also known as Keller's $C_{c}^{k}$-theory [19]. For $C^{\alpha}$-maps, see [1] (cf. [3] and [15] for the case of two variables, $\left.\alpha \in\left(\mathbb{N}_{0} \cup\{\infty\}\right)^{2}\right)$. We now introduce concepts for later use and collect basic facts. For proofs, see "Appendix".

2.1 Consider locally convex spaces $E, F$ and a map $f: U \rightarrow F$ on an open subset $U \subseteq E$. Write

$$
\left(D_{y} f\right)(x):=\left.\frac{\mathrm{d}}{\mathrm{d} t}\right|_{t=0} f(x+t y)
$$

for the directional derivative of $f$ at $x \in U$ in the direction $y \in E$, if it exists. Let $k \in \mathbb{N}_{0} \cup\{\infty\}$. If $f$ is continuous, the iterated directional derivatives

$$
d^{j} f\left(x, y_{1}, \ldots, y_{j}\right):=\left(D_{y_{j}} \ldots D_{y_{1}} f\right)(x)
$$

exist for all $j \in \mathbb{N}_{0}$ such that $j \leq k, x \in U$ and $y_{1}, \ldots, y_{j} \in E$, and the maps $d^{j} f: U \times$ $E^{j} \rightarrow F$ are continuous, then $f$ is called $C^{k}$. If $U$ may not be open, but has dense interior $U^{o}$ and is locally convex in the sense that each $x \in U$ has a convex neighborhood in $U$, following [15] a map $f: U \rightarrow F$ is called $C^{k}$ if it is continuous, $\left.f\right|_{U^{o}}$ is $C^{k}$ and $d^{j}\left(\left.f\right|_{U^{o}}\right)$ has a continuous extension $d^{j} f: U \times E^{j} \rightarrow F$ for all $j \in \mathbb{N}_{0}$ with $j \leq k$. The $C^{\infty}$-maps are also called smooth.

Remark 2.2 If $E=\mathbb{R}^{n}$ and $U$ is relatively open in [0, $\infty{ }^{n}$, then $f$ as above is $C^{k}$ if and only $f$ has a $C^{k}$-extension to an open set in $\mathbb{R}^{n}$ (see [13], cf. [17]).

2.3 Let $k \in \mathbb{N} \cup\{\infty\}$. A manifold with rough boundary modeled on a non-empty set $\mathcal{E}$ of locally convex spaces is a Hausdorff topological space $M$, together with a set $\mathcal{A}$ of homeomorphisms ("charts") $\phi: U_{\phi} \rightarrow V_{\phi}$ from an open subset $U_{\phi} \subseteq M$ onto a locally convex subset $V_{\phi} \subseteq E_{\phi}$ with dense interior for some $E_{\phi} \in \mathcal{E}$, such that $\phi \circ \psi^{-1}$ is $C^{k}$ for all $\phi, \psi \in \mathcal{A}$, the union $\bigcup_{\phi \in \mathcal{A}} U_{\phi}$ equals $M$, and $\mathcal{A}$ is maximal. If $k=0$, assume in addition that $\phi(x) \in \partial V_{\phi}$ if and only if $\psi(x) \in \partial V_{\psi}$ for all $\phi, \psi \in \mathcal{A}$ with $x \in U_{\phi} \cap U_{\psi}$ (which is automatic if $k \geq 1$ ). Let $\partial M$ be the set of all $x \in M$ such that $\phi(x) \in \partial V_{\phi}$ for some (and hence any) chart $\phi$ around $x$. If $\mathcal{E}$ is a singleton, $M$ is called pure. If $M$ is a $C^{k}$-manifold with rough boundary and $\partial M=\emptyset$, then $M$ is called a $C^{k}$-manifold or a $C^{k}$-manifold without boundary, for emphasis. (See [15] for all of this in the pure case; cf. [4] for modifications in the general case).

2.4 All manifolds and Lie groups considered in the article are modeled on locally convex spaces which may be infinite-dimensional, unless the contrary is stated. Finite-dimensional manifolds need not be paracompact or $\sigma$-compact, unless stated explicitly. As we are interested in manifolds of mappings, consideration of pure manifolds would not be sufficient. 
2.5 If $U$ is an open subset of a locally convex space $E$ (or a locally convex subset with dense interior), we identify its tangent bundle $T U$ with $U \times E$, as usual, with bundle projection $(x, y) \mapsto x$. If $M$ is a $C^{k}$-manifold with rough boundary and $f: M \rightarrow U$ a $C^{k}$-map with $k \geq 1$, we write $d f$ for the second component of $T f: T M \rightarrow T U=U \times E$. Thus, $T f=\left(f \circ \pi_{T M}, d f\right)$, using the bundle projection $\pi_{T M}: T M \rightarrow M$.

2.6 If $G$ is a Lie group with neutral element $e$, we write $L(G):=T_{e} G$ (or $\mathfrak{g}$ ) for its tangent space at $e$, endowed with its natural topological Lie algebra structure. If $\psi: G \rightarrow H$ is a smooth homomorphism between Lie groups, we let $L(\psi):=T_{e} \psi: L(G) \rightarrow L(H)$ be the associated continuous Lie algebra homomorphism.

2.7 If $G$ is a Lie group with Lie algebra $\mathfrak{g}$ and $I$ a non-degenerate interval with $0 \in I$, we define $\delta^{\ell}(\eta)$ for $\eta \in C^{1}(I, G)$ via $\delta^{\ell}(\eta)(t):=\eta(t)^{-1} . \dot{\eta}(t)$, with $\dot{\eta}(t):=T \eta(t, 1)$.

Lemma 2.8 Let $k, r \in \mathbb{N}_{0} \cup\{\infty\}$ with $k \geq r$. If $G$ is $C^{r}$-semiregular and $\gamma \in C^{k}(I, \mathfrak{g})$, then there exists a unique $\eta \in C^{1}(I, \mathfrak{g})$ such that $\eta(0)=e$ and $\delta^{\ell}(\eta)=\gamma$. Moreover, $\eta$ is $C^{k+1}$.

2.9 Let $M$ be a smooth manifold (without boundary). A subset $N \subseteq M$ is called a submanifold if, for each $x \in N$, there exist a chart $\phi: U_{\phi} \rightarrow V_{\phi} \subseteq E_{\phi}$ of $M$ around $x$ and a closed vector subspace $F \subseteq E_{\phi}$ such that $\phi\left(U_{\phi} \cap N\right)=V_{\phi} \cap F$.

2.10 Let $M$ be a smooth manifold with rough boundary. A subset $N \subseteq M$ is called a full submanifold if, for each $x \in N$, there exists a chart $\phi: U_{\phi} \rightarrow V_{\phi} \subseteq E_{\phi}$ of $M$ around $x$ such that $\phi\left(U_{\phi} \cap N\right)$ is a locally convex subset of $E_{\phi}$ with dense interior.

2.11 Let $F$ and $E_{1}, \ldots, E_{n}$ be locally convex spaces, $U_{j} \subseteq E_{j}$ be an open subset for $j \in$ $\{1, \ldots, n\}$ and $f: U \rightarrow F$ be a map on $U:=U_{1} \times \cdots \times U_{n}$. Identifying $E:=E_{1} \times \cdots \times E_{n}$ with $E_{1} \oplus \cdots \oplus E_{n}$, we can identify each $E_{j}$ with a vector subspace of $E$, and simply write $D_{y} f(x)$ for a directional derivative with $x \in U, y \in E_{j}$ (rather than $D_{(0, \ldots, 0, y, 0, \ldots, 0)} f(x)$ with $j-1$ zeros on the left and $n-j$ zeros on the right-hand side). For $y=\left(y_{1}, \ldots, y_{k}\right) \in E_{j}^{k}$, abbreviate

$$
D_{y}:=D_{y_{k}} \ldots D_{y_{1}} .
$$

Let $\alpha \in\left(\mathbb{N}_{0} \cup\{\infty\}\right)^{n}$. Following [1], we say that $f$ is $C^{\alpha}$ if $f$ is continuous, the iterated directional derivatives

$$
d^{\beta} f\left(x, y_{1}, \ldots, y_{n}\right):=\left(D_{y_{n}} \cdots D_{y_{1}} f\right)(x)
$$

exist for all $\beta \in \mathbb{N}_{0}^{n}$ with $\beta \leq \alpha, x \in U$ and $y_{j}=\left(y_{j, 1}, \ldots, y_{j, \beta_{j}}\right) \in\left(E_{j}\right)^{\beta_{j}}$ for $j \in$ $\{1, \ldots, n\}$, and

$$
d^{\beta} f: U \times E_{1}^{\beta_{1}} \times \cdots \times E_{n}^{\beta_{n}} \rightarrow F
$$

is continuous. If $U_{j}$ may not be open but is a locally convex subset of $E_{j}$ with dense interior, we say that $f: U \rightarrow F$ is $C^{\alpha}$ if $f$ is continuous, $\left.f\right|_{U^{o}}$ is $C^{\alpha}$ and $d^{\beta}\left(\left.f\right|_{U^{o}}\right)$ has a continuous extension $d^{\beta} f: U \times E_{1}^{\beta_{1}} \times \cdots \times E_{n}^{\beta_{n}} \rightarrow F$ for all $\beta \in\left(\mathbb{N}_{0}\right)^{n}$ such that $\beta \leq \alpha$.

2.12 Let $M_{1}, \ldots, M_{n}$ be $C^{\infty}$-manifolds with rough boundary, $\alpha \in\left(\mathbb{N}_{0} \cup\{\infty\}\right)^{n}$ and $N$ be a $C^{k}$-manifold with $k \geq|\alpha|$. We say that a map $f: M_{1} \times \cdots \times M_{n} \rightarrow N$ is $C^{\alpha}$ if, for each $x=\left(x_{1}, \ldots, x_{n}\right) \in M_{1} \times \cdots \times M_{n}$, there are charts $\phi_{j}: U_{j} \rightarrow V_{j}$ for $M_{j}$ around $x_{j}$ for $j \in\{1, \ldots, n\}$ and a chart $\psi: U_{\psi} \rightarrow V_{\psi}$ for $n$ around $f(x)$ such that $f\left(U_{1} \times \cdots \times U_{n}\right) \subseteq U_{\psi}$ and

$$
\psi \circ f \circ\left(\phi_{1} \times \cdots \times \phi_{n}\right)^{-1}: V_{1} \times \cdots \times V_{n} \rightarrow V_{\psi}
$$


is $C^{\alpha}$. The latter then holds for any such charts, by the chain rule for $C^{\alpha}$-maps (as in [1, Lemma 3.16]).

2.13 Let $N$ and $M_{1}, \ldots, M_{n}$ be $C^{\infty}$-manifolds with rough boundary, $\sigma$ be a permutation of $\{1, \ldots, n\}$, and $\alpha \in\left(\mathbb{N}_{0} \cup\{\infty\}\right)^{n}$. If $f: M_{\sigma(1)} \times \cdots \times M_{\sigma(n)} \rightarrow N$ is $C^{\alpha \circ \sigma}$, then the map

$$
M_{1} \times \cdots \times M_{n} \rightarrow N, \quad\left(x_{1}, \ldots, x_{n}\right) \mapsto f\left(x_{\sigma(1)}, \ldots, x_{\sigma(n)}\right)
$$

is $C^{\alpha}$. This follows from Schwarz' Theorem (in the form of [1, Proposition 3.5]).

We shall use simple facts:

Lemma 2.14 Let $E_{j}$ for $j \in\{1, \ldots, n\}$ and $F$ be locally convex spaces, and $U_{j} \subseteq E_{j}$ be a locally convex subset with dense interior. Let $E:=E_{1} \times \cdots \times E_{n}, U:=U_{1} \times \cdots \times U_{n}$, $\alpha \in\left(\mathbb{N}_{0} \cup\{\infty\}\right)^{n}$ and $f: U \rightarrow F$ be a map.

(a) If $Y \subseteq F$ is a closed vector subspace and $f(U) \subseteq Y$, then $f$ is $C^{\alpha}$ if and only if its co-restriction $\left.f\right|^{Y}: U \rightarrow Y$ is $C^{\alpha}$.

(b) If $F$ is the projective limit of a projective system $\left(\left(F_{a}\right)_{a \in A},\left(\lambda_{a, b}\right)_{a \leq b}\right)$ of locally convex spaces $F_{a}$ and continuous linear maps $\lambda_{a, b}: F_{b} \rightarrow F_{a}$, with limit maps $\lambda_{a}: F \rightarrow F_{a}$, then $f$ is $C^{\alpha}$ if and only if $\lambda_{a} \circ f: U \rightarrow F_{a}$ is $C^{\alpha}$ for all $a \in A$.

Lemma 2.15 Let $M, N$, and $L_{1}, \ldots, L_{n}$ be smooth manifolds with rough boundary, $F$ be a locally convex space, $\psi: M \rightarrow F \times N$ be a $C^{\infty}$-diffeomorphism, and $f: L_{1} \times \cdots \times L_{n} \rightarrow M$ be a map. Assume that $F$ is the projective limit of a projective system $\left(\left(F_{a}\right)_{a \in A},\left(\lambda_{a, b}\right)_{a \leq b}\right)$ of locally convex spaces $F_{a}$ and continuous linear mappings $\lambda_{a, b}: F_{b} \rightarrow F_{a}$, with limit maps $\lambda_{a}: F \rightarrow F_{a}$. For $a \in A$, let $M_{a}$ be a smooth manifold and $\rho_{a}: M \rightarrow M_{a}$ be a $C^{\infty}$-map. Assume that there exist $C^{\infty}$-maps $\psi_{a}: M_{a} \rightarrow F_{a} \times N$ making the diagram

$$
\begin{aligned}
M & \stackrel{\psi}{\longrightarrow} F \times N \\
\rho_{a} \downarrow & \\
M_{a} \stackrel{\psi_{a}}{\longrightarrow} F_{a} & \times N
\end{aligned}
$$

commute. Then, $f$ is $C^{\alpha}$ if and only if $\rho_{a} \circ f$ is $C^{\alpha}$ for all $a \in A$.

2.16 If $\alpha=\left(\alpha_{1}, \ldots, \alpha_{n}\right) \in\left(\mathbb{N}_{0} \cup\{\infty\}\right)^{n}$ and $\beta=\left(\beta_{1}, \ldots, \beta_{m}\right) \in\left(\mathbb{N}_{0} \cup\{\infty\}\right)^{m}$, we shall write $(\alpha, \beta)$ as a shorthand for $\left(\alpha_{1}, \ldots, \alpha_{n}, \beta_{1}, \ldots, \beta_{m}\right)$ and abbreviate $C^{(\alpha, \beta)}$ as $C^{\alpha, \beta}$. Likewise for higher numbers of multiindices.

Let $r \in \mathbb{N}_{0} \cup\{\infty\}, E_{1}, \ldots, E_{n}$ and $F$ be locally convex spaces and $U_{j}$ be a locally convex subset of $E_{j}$ with dense interior, for $j \in\{1, \ldots, n\}$. We mention that a map $f: U_{1} \times \cdots \times$ $U_{n} \rightarrow F$ is $C^{r}$ if and only if it is $C^{\beta}$ for all $\beta \in\left(\mathbb{N}_{0} \cup\{\infty\}\right)^{n}$ such that $|\beta| \leq r$. More generally, the following is known (as first formulated and proved in the unpublished work [18]):

Lemma 2.17 For $i \in\{1, \ldots, n\}$, let $E_{i}$ be a locally convex space of the form $E_{i}=E_{i, 1} \times$ $\cdots \times E_{i, m_{i}}$ for some $m_{i} \in \mathbb{N}$ and locally convex spaces $E_{i, 1}, \ldots, E_{i, m_{i}}$. Let $U_{i, j}$ be a locally convex subset of $E_{i, j}$ with dense interior for all $i \in\{1, \ldots, n\}$ and $j \in\left\{1, \ldots, m_{i}\right\}$; define $U_{i}:=U_{i, 1} \times \cdots \times U_{i, m_{i}}$. Let $\alpha \in\left(\mathbb{N}_{0} \cup\{\infty\}\right)^{n}$. Then, a map $f: U_{1} \times \cdots \times U_{n} \rightarrow F$ is $C^{\alpha}$ if and only if $f$ is $C^{\beta_{1}, \ldots, \beta_{n}}$ on $\prod_{i=1}^{n} \prod_{j=1}^{m_{i}} U_{i, j}$ for all $\left(\beta_{1}, \ldots, \beta_{n}\right) \in \prod_{i=1}^{n}\left(\mathbb{N}_{0} \cup\{\infty\}\right)^{m_{i}}$ such that $\left|\beta_{i}\right| \leq \alpha_{i}$ for all $i \in\{1, \ldots, n\}$. 


\section{The compact-open $C^{\alpha}$-topology}

As a further preliminary, we introduce a topology on $C^{\alpha}\left(M_{1} \times \cdots \times M_{n}, N\right)$ which parallels the familiar compact-open $C^{k}$-topology on $C^{k}(M, N)$. Basic properties are recorded, with proofs in "Appendix A".

As usual, $T^{0} M:=M, T^{1} M:=T M$ and $T^{k} M:=T\left(T^{k-1} M\right)$ for a smooth manifold $M$ with rough boundary and integers $k \geq 2$ (see [15]).

3.1 In 3.2-3.10, $M_{1}, \ldots, M_{n}$ will be smooth manifolds with rough boundary, and $M:=$ $M_{1} \times \cdots \times M_{n}$. In 3.3-3.9, we let $N$ be a smooth manifold with rough boundary and $\alpha \in\left(\mathbb{N}_{0} \cup\{\infty\}\right)^{n}$.

3.2 We define the $\beta$-tangent bundle of $M$ as $T^{\beta} M:=T^{\beta_{1}} M_{1} \times \cdots \times T^{\beta_{n}} M_{n}$ for $\beta=$ $\left(\beta_{1}, \ldots, \beta_{n}\right) \in\left(\mathbb{N}_{0}\right)^{n}$.

3.3 Let $f: M \rightarrow N$ be a $C^{\alpha}$-map. For $\beta=\left(\beta_{1}, \ldots, \beta_{n}\right) \in\left(\mathbb{N}_{0}\right)^{n}$ with $\beta \leq \alpha$, we define

$$
T^{\beta} f: T^{\beta} M \rightarrow T^{|\beta|} N
$$

recursively, as follows: We first note that, by Lemma A.1,

$$
T^{\left(0, \ldots, 0, \beta_{n}\right)} f: M_{1} \times \cdots \times M_{n-1} \times T^{\beta_{n}} M_{n} \rightarrow T^{\beta_{n}} N,
$$

$\left(x_{1}, \ldots, x_{n-1}, v_{n}\right) \mapsto T^{\beta_{n}}\left(f\left(x_{1}, \ldots, x_{n-1}, \cdot\right)\right)\left(v_{n}\right)$ is a $C^{\left(\alpha_{1}, \ldots, \alpha_{n-1}, 0\right)}$-map. If a $C^{\left(\alpha_{1}, \ldots, \alpha_{k-1}, 0, \ldots, 0\right)}$-map $g:=T^{\left(0, \ldots, 0, \beta_{k}, \ldots, \beta_{n}\right)} f: T^{\left(0, \ldots, 0, \beta_{k}, \ldots, \beta_{n}\right)} M \rightarrow T^{\beta_{k}+\cdots+\beta_{n}} N$ has already been constructed for $k \in\{2, \ldots, n\}$, then the map

$$
T^{\left(0, \ldots, 0, \beta_{k-1}, \ldots, \beta_{n}\right)} f: T^{\left(0, \ldots, 0, \beta_{k-1}, \ldots, \beta_{n}\right)} M \rightarrow T^{\beta_{k-1}+\cdots+\beta_{n}} N
$$

taking $\left(x_{1}, \ldots, x_{k-2}, v_{k-1}, \ldots, v_{n}\right)$ to $T^{\beta_{k-1}}\left(g\left(x_{1}, \ldots, x_{k-2}, \cdot, v_{k}, \ldots, v_{n}\right)\right)\left(v_{k-1}\right)$ is a $C^{\left(\alpha_{1}, \ldots, \alpha_{k-2}, 0, \ldots, 0\right)}$-map (see Lemmas 2.13 and A.1).

Definition 3.4 The compact-open $C^{\alpha}$-topology on $C^{\alpha}(M, N)$ is the initial topology with respect to the mappings

$$
T^{\beta}: C^{\alpha}(M, N) \rightarrow C\left(T^{\beta} M, T^{|\beta|} N\right), \quad f \mapsto T^{\beta} f
$$

for $\beta \in\left(\mathbb{N}_{0}\right)^{n}$ with $\beta \leq \alpha$, using the compact-open topology on $C\left(T^{\beta} M, T^{|\beta|} N\right)$.

Pushforwards and pullbacks are continuous.

Lemma 3.5 Using compact-open $C^{\alpha}$-topologies, we have:

(a) If $L$ is a smooth manifold with rough boundary and $g: N \rightarrow L$ a smooth map, then the following map is continuous:

$$
g_{*}:=C^{\alpha}(M, g): C^{\alpha}(M, N) \rightarrow C^{\alpha}(M, L), \quad f \mapsto g \circ f .
$$

(b) Let $L_{j}$ be a smooth manifold with rough boundary for $j \in\{1, \ldots, n\}$ and $g_{j}: L_{j} \rightarrow M_{j}$ be a smooth map. Abbreviate $L:=L_{1} \times \cdots \times L_{n}$ and $g:=g_{1} \times \cdots \times g_{n}$. Then, the following map is continuous:

$$
g^{*}:=C^{\alpha}(g, N): C^{\alpha}(M, N) \rightarrow C^{\alpha}(L, N), \quad f \mapsto f \circ g .
$$


Remark 3.6 If $L_{j}$ is a full submanifold of $M_{j}$ for $j \in\{1, \ldots, m\}$, then the inclusion map $g_{j}: L_{j} \rightarrow M_{j}, x \mapsto x$ is smooth. By Lemma $3.5(\mathrm{~b})$, the map

$$
\rho:=C^{\alpha}\left(g_{1} \times \cdots \times g_{n}, N\right): C^{\alpha}(M, N) \rightarrow C^{\alpha}(L, N)
$$

is continuous, which is the restriction map $C^{\alpha}(M, N) \rightarrow C^{\alpha}(L, N),\left.f \mapsto f\right|_{L}$.

Lemma 3.7 Let $\left(K_{i}\right)_{i \in I}$ be a family of subsets $K_{i} \subseteq M$ whose interiors $K_{i}^{o}$ cover $M$, such that $K_{i}=K_{i, 1} \times \cdots \times K_{i, n}$ for certain full submanifolds $K_{i, j} \subseteq M_{j}$ for $j \in\{1, \ldots, n\}$. Then, the compact-open $C^{\alpha}$-topology on $C^{\alpha}(M, N)$ is initial with respect to the restriction maps $C^{\alpha}(M, N) \rightarrow C^{\alpha}\left(K_{i}, N\right)$ for $i \in I$.

Lemma 3.8 For $j \in\{1, \ldots, n\}$, let $S_{j}$ be a full submanifold of $M_{j}$. Abbreviate $S:=S_{1} \times$ $\cdots \times S_{n}$. Then, $T^{\beta} S$ is a full submanifold of $T^{\beta} M$ for all $\beta \in\left(\mathbb{N}_{0}\right)^{n}$, and the smooth manifold structure on $T^{\beta} S$ as the $\beta$-tangent bundle of $S$ coincides with the smooth manifold structure as a full submanifold of $T^{\beta} M$. Analogous conclusions (with submanifolds in place of full submanifolds) hold if $\partial M_{j}=\emptyset$ for all $j \in\{1, \ldots, n\}$ and $S_{j} \subseteq M_{j}$ is a submanifold.

Lemma 3.9 If $S$ is a full submanifold of $N$ or $\partial N=\emptyset$ and $S \subseteq N$ is a submanifold, then the compact-open $C^{\alpha}$-topology on $C^{\alpha}(M, S)$ coincides with the topology on $C^{\alpha}(M, S)$ induced by $C^{\alpha}(M, N)$.

Lemma 3.10 If $F$ is a locally convex space, then $C^{\alpha}(M, F)$ is a vector subspace of $F^{M}$. The compact-open $C^{\alpha}$-topology makes $C^{\alpha}(M, F)$ a locally convex space.

Lemma 3.11 Let $M_{1}, \ldots, M_{n}$ be smooth manifolds with rough boundary, $M:=M_{1} \times \cdots \times$ $M_{n}$, and $\alpha \in\left(\mathbb{N}_{0} \cup\{\infty\}\right)^{n}$.

(a) If $F$ is a locally convex space whose topology is initial with respect to a family $\left(\lambda_{i}\right)_{i \in I}$ of linear mappings $\lambda_{i}: F \rightarrow F_{i}$ to locally convex spaces $F_{i}$, then the compact-open $C^{\alpha}$-topology on $C^{\alpha}(M, F)$ is initial with respect to the $\left(\lambda_{i}\right)_{*}: C^{\alpha}(M, F) \rightarrow C^{\alpha}\left(M, F_{i}\right)$ for $i \in I . i$.

(b) If $F$ is a locally convex space and $F=\prod_{i \in I} F_{i}$ for a family $\left(F_{i}\right)_{i \in I}$ of locally convex spaces, let $\mathrm{pr}_{i}: F \rightarrow F_{i}$ be the projection onto the ith component and $\left(\mathrm{pr}_{i}\right)_{*}: C^{\alpha}(M, F) \rightarrow C^{\alpha}\left(M, F_{i}\right)$. Then,

$$
\Theta:=\left(\left(\mathrm{pr}_{i}\right)_{*}\right)_{i \in I}: C^{\alpha}(M, F) \rightarrow \prod_{i \in I} C^{\alpha}\left(M, F_{i}\right)
$$

is an isomorphism of topological vector spaces.

(c) Assume that all of $M_{1}, \ldots, M_{n}$ are locally compact. Let $N_{i}$ be a smooth manifold with rough boundary for $i \in\{1,2\}$ and $\mathrm{pr}_{i}: N_{1} \times N_{2} \rightarrow N_{i}$ be the projection onto the ith component. Using the compact-open $C^{\alpha}$-topology on sets of $C^{\alpha}$-maps, we get a homeomorphism

$$
\Psi:=\left(\left(\mathrm{pr}_{1}\right)_{*},\left(\mathrm{pr}_{2}\right)_{*}\right): C^{\alpha}\left(M, N_{1} \times N_{2}\right) \rightarrow C^{\alpha}\left(M, N_{1}\right) \times C^{\alpha}\left(M, N_{2}\right) .
$$

Using the multiplication $\mathbb{R} \times T N \rightarrow T N,(t, v) \mapsto t v$ with scalars, we have:

Lemma 3.12 Let $M_{1}, \ldots, M_{n}$ be locally compact smooth manifolds with rough boundary, $M:=M_{1} \times \cdots \times M_{n}, \alpha \in\left(\mathbb{N}_{0} \cup\{\infty\}\right)^{n}$, and $N$ be a smooth manifold with rough boundary. Then, the map

$$
\mu: C^{\alpha}(M, \mathbb{R}) \times C^{\alpha}(M, T N) \rightarrow C^{\alpha}(M, T N)
$$

determined by $\mu(f, g)(x):=f(x) g(x)$ is continuous. 
In [1], exponential laws were provided for function spaces on products of pure manifolds. The one we need remains valid for manifolds which need not be pure:

Lemma 3.13 Let $N_{1}, \ldots, N_{m}$ and $M_{1}, \ldots, M_{n}$ be smooth manifolds with rough boundary (none of which needs to be pure). Let $\alpha \in\left(\mathbb{N}_{0} \cup\{\infty\}\right)^{m}, \beta \in\left(\mathbb{N}_{0} \cup\{\infty\}\right)^{n}$ and $E$ be a locally convex space. Abbreviate $N:=N_{1} \times \cdots \times N_{m}$ and $M:=M_{1} \times \cdots \times M_{n}$. For $f \in C^{\alpha, \beta}(N \times M, E)$, we then have $f_{x}:=f(x, \cdot) \in C^{\beta}(M, E)$ for each $x \in N$ and the map $f^{\vee}: N \rightarrow C^{\beta}(M, E), x \mapsto f_{x}$ is $C^{\alpha}$. The map

$$
\Phi: C^{\alpha, \beta}(N \times M, E) \rightarrow C^{\alpha}\left(N, C^{\beta}(M, E)\right), \quad f \mapsto f^{\vee}
$$

is linear and a homeomorphism onto its image. If $M_{j}$ is locally compact for all $j \in\{1, \ldots, n\}$, then $\Phi$ is a homeomorphism. The inverse map $\Phi^{-1}$ sends $g \in C^{\alpha}\left(N, C^{\beta}(M, E)\right)$ to the map $g^{\wedge}$ defined via $g^{\wedge}(x, y):=g(x)(y)$.

The next lemma describes the $C^{\alpha}$-topology on $C^{\alpha}(U, F)$ more explicitly. It will not be used here. The proof, which parallels the $C^{k}$-case in [15, Lemma 4.1.12], can be found in the preprint version of this article, arXiv:2109.01804.

Lemma 3.14 Let $E_{j}$ be a locally convex space for $j \in\{1, \ldots, n\}$ and $U_{j} \subseteq E_{j}$ be a locally convex subset with dense interior. Let $F$ be a locally convex space, $\alpha \in\left(\mathbb{N}_{0} \cup\{\infty\}\right)^{n}$, and $U:=U_{1} \times \cdots \times U_{n}$. Then, the compact-open $C^{\alpha}$-topology on $C^{\alpha}(U, F)$ is initial with respect to the maps

$$
d^{\beta}: C^{\alpha}(U, F) \rightarrow C\left(U \times E_{1}^{\beta_{1}} \times \cdots \times E_{n}^{\beta_{n}}, F\right), \quad f \mapsto d^{\beta} f
$$

for $\beta \in\left(\mathbb{N}_{0}\right)^{n}$ with $\beta \leq \alpha$, using the compact-open topology on the ranges.

\section{4 (Pre-)Canonical manifold structures}

In this section, we establish basic properties of canonical manifolds of mappings, and precanonical ones. We begin with examples.

Example 4.1 Let $n \in \mathbb{N}$ and $\alpha \in\left(\mathbb{N}_{0} \cup\{\infty\}\right)^{n}$.

(a) Let $M_{1}, \ldots, M_{n}$ be locally compact smooth manifolds with rough boundary and $E$ a locally convex space. Then, $C^{\alpha}\left(M_{1} \times \cdots \times M_{n}, E\right)$ is a canonical manifold due to Lemma 3.13. The same holds for $C^{\alpha}\left(M_{1} \times \cdots \times M_{n}, N\right)$ if $N$ is a smooth manifold diffeomorphic to $E$, endowed with the $C^{\infty}$-manifold structure making $\varphi_{*}: C^{\alpha}(M, N) \rightarrow$ $C^{\alpha}(M, E)$ a diffeomorphism, where $\varphi: E \rightarrow N$ is a $C^{\infty}$-diffeomorphism.

(b) Familiar examples of mapping groups turn out to be canonical, notably loop groups $C^{k}\left(\mathbb{S}^{1}, G\right)$ for $G$ a Lie group, and certain Lie groups of the form $C^{k}(\mathbb{R}, G)$ discussed in $[2,24]$. We extend these constructions in Sect. 6.

We will now establish general properties of canonical manifolds.

4.2 Conventions We denote by $\alpha, \beta$ multiindices in $\left(\mathbb{N}_{0} \cup\{\infty\}\right)^{n}$ for some $n \in \mathbb{N}$. Likewise we will usually adopt the shorthand $M:=M_{1} \times M_{2} \times \cdots \times M_{n}$ where the $M_{i}$ are locally compact manifolds (possibly with rough boundary). If $M$ is the domain of definition of the function space $C^{\alpha}(M, N)$ we will assume that the number of entries of the multiindex $\alpha$ coincides with the number of factors in the product $M$. 
Lemma 4.3 If $C^{\alpha}(M, N)$ is endowed with a pre-canonical manifold structure, then the following holds:

(a) The evaluation map ev: $C^{\alpha}(M, N) \times M \rightarrow N$, ev $(\gamma, x):=\gamma(x)$ is $C^{\infty, \alpha}$.

(b) Pre-canonical manifold structures are unique in the following sense: If we write $C^{\alpha}$ $(M, N)^{\prime}$ for $C^{\alpha}(M, N)$ with another pre-canonical manifold structure, then id: $C^{\alpha}(M, N) \rightarrow C^{\alpha}(M, N)^{\prime}, \gamma \mapsto \gamma$ is a $C^{\infty}$-diffeomorphism.

(c) Let $S \subseteq N$ be a submanifold such that the set $C^{\alpha}(M, S)$ is a submanifold of $C^{\alpha}(M, N)$. Then, the submanifold structure on $C^{\alpha}(M, S)$ is pre-canonical.

Proof (a) Since id: $C^{\alpha}(M, N) \rightarrow C^{\alpha}(M, N)$ is $C^{\infty}$ and $C^{\alpha}(M, N)$ is endowed with a pre-canonical manifold structure, it follows that $\mathrm{id}^{\wedge}: C^{\alpha}(M, N) \times M \rightarrow N,(\gamma, x) \mapsto$ $\operatorname{id}(\gamma)(x)=\gamma(x)=\operatorname{ev}(\gamma, x)$ is $C^{\infty, \alpha}$.

(b) The map $f:=\mathrm{id}: C^{\alpha}(M, N) \rightarrow C^{\alpha}(M, N)^{\prime}$ satisfies $f^{\wedge}=\mathrm{ev}$ where ev: $C^{\alpha}(M, N) \times$ $M \rightarrow N$ is $C^{\infty, \alpha}$, by (a). Since $C^{\alpha}(M . N)^{\prime}$ is endowed with a pre-canonical manifold structure, it follows that $f$ is $C^{\infty}$. By the same reasoning, $f^{-1}=\mathrm{id}: C^{\alpha}(M, N)^{\prime} \rightarrow$ $C^{\alpha}(M, N)$ is $C^{\infty}$.

(c) As $C^{\alpha}(M, S)$ is a submanifold of $C^{\alpha}(M, N)$, the inclusion $\iota: C^{\alpha}(M, S) \rightarrow C^{\alpha}(M, N)$ is $C^{\infty}$. Likewise, the inclusion map $j: S \rightarrow N$ is $C^{\infty}$. Let $L=L_{1} \times \cdots \times L_{k}$ be a product of smooth manifolds (possibly with rough boundary) modeled on locally convex spaces and $f: L \rightarrow C^{\alpha}(M, S)$ be a map. If $f$ is $C^{\beta}$, then $\iota \circ f$ is $C^{\beta}$, entailing that $(\iota \circ f)^{\wedge}: L \times M \rightarrow N,(x, y) \mapsto f(x)(y)$ is $C^{\beta, \alpha}$. As the image of this map is contained in $S$, which is a submanifold of $N$, we deduce that $f^{\wedge}=\left.(\iota \circ f)^{\wedge}\right|^{S}$ is $C^{\beta, \alpha}$. For the converse, assume that $f^{\wedge}: L \times M \rightarrow S$ is $C^{\beta, \alpha}$. Then also, $(\iota \circ f)^{\wedge}=j \circ\left(f^{\wedge}\right): L \times M \rightarrow$ $N$ is $C^{\beta, \alpha}$. Hence, $\iota \circ f: L \rightarrow C^{\alpha}(M, N)$ is $C^{\beta}$ (the manifold structure on the range being pre-canonical). As $\iota \circ f$ is a $C^{\beta}$-map with image in $C^{\alpha}(M, S)$ which is a submanifold of $C^{\alpha}(M, N)$, we deduce that $f$ is $C^{\beta}$.

Remark 4.4 Note that due to Lemma 4.3(a), the evaluation on a canonical manifold is a $C^{\infty, \alpha}$-map whence it is at least continuous. For a $C^{k}$-manifold $M$ which is $C^{k}$-regular ${ }^{1}$ and a locally convex space $E \neq\{0\}$, it is well known that for the compact-open $C^{k}$-topology the evaluation ev: $C^{k}(M, E) \times M \rightarrow E$ is continuous if and only if $M$ is locally compact. A similar statement holds for the compact-open $C^{\alpha}$-topology. Using a chart for $N$ and cutoff functions, we deduce that the evaluation of $C^{\alpha}(M, N)$ is discontinuous if $M$ fails to be locally compact, provided $N$ is not discrete and $M$ is $C^{|\alpha|}$-regular; then $C^{\alpha}(M, N)$ cannot admit a canonical manifold structure.

We now turn to smoothness properties of the composition map.

Lemma 4.5 Assume that $C^{|\alpha|+s}(N, L), C^{\alpha}(M, N)$, and $C^{\alpha}(M, L)$ are endowed with precanonical manifold structures. Then, the composition map

$$
\text { comp: } C^{|\alpha|+s}(N, L) \times C^{\alpha}(M, N) \rightarrow C^{\alpha}(M, L), \quad(f, g) \mapsto f \circ g
$$

is a $C^{\infty, s}$-map, for every $s \in \mathbb{N}_{0} \cup\{\infty\}$.

Proof Since $C^{\alpha}(M, L)$ is pre-canonical, comp is $C^{\infty, s}$ if and only if

$$
\operatorname{comp}^{\wedge}: C^{|\alpha|+s}(N, L) \times C^{\alpha}(M, N) \times M \rightarrow L, \quad(f, g, x) \mapsto f(g(x))
$$

${ }_{1}^{1}$ Meaning that the topology on $M$ is initial with respect to $C^{k}(M, \mathbb{R})$. This holds if $M$ is a regular topological space and all modeling spaces are $C^{k}$-regular, see [15]. 
is a $C^{\infty, s, \alpha}$-map. The formula shows that $\operatorname{comp}^{\wedge}(f, g, x)=\operatorname{ev}(f, \operatorname{ev}(g, x))$, where the outer evaluation map is $C^{\infty,|\alpha|+s}$ and the inner one $C^{\infty, \alpha}$, by Lemma 4.3 (a), as $C^{|\alpha|+s}(N, L)$ and $C^{\alpha}(M, N)$ are pre-canonical manifolds. Using the chain rule [1, Lemma 3.16], we deduce that comp $^{\wedge}$ is $C^{\infty, s, \alpha}$.

Corollary 4.6 If $C^{\alpha}(M, N)$ and $C^{\alpha}(M, L)$ are endowed with pre-canonical manifold structures, then the pushforward $f_{*}: C^{\alpha}(M, N) \rightarrow C^{\alpha}(M, L), g \mapsto f \circ g$ is a $C^{s}$-map for every $f \in C^{|\alpha|+s}(N, L)$.

Corollary 4.7 Let $C^{|\alpha|+s}(N, L)$ and $C^{\alpha}(M, L)$ be endowed with pre-canonical manifold structures. For a $C^{\alpha}$-map $g: M \rightarrow N$ the pullback $g^{*}: C^{|\alpha|+s}(N, L) \rightarrow C^{\alpha}(M, L), f \mapsto$ $f \circ g$ is smooth for every $s \in \mathbb{N}_{0}$.

The chain rule also allows the following result to be deduced.

Lemma 4.8 Let $C^{\alpha}(M, N)$ and $C^{\alpha}(L, N)$ be endowed with pre-canonical manifold structures where $\alpha=\left(\alpha_{1}, \ldots, \alpha_{n}\right), M=M_{1} \times \cdots \times M_{n}$ and $L=L_{1} \times \cdots \times L_{n}$. Assume that $g_{i}: L_{i} \rightarrow M_{i}$ is a $C^{\alpha_{i}}$-map for $i \in\{1, \ldots, n\}$. Then, the pullback

$$
g^{*}: C^{\alpha}(M, N) \rightarrow C^{\alpha}(L, N), f \mapsto f \circ\left(g_{1} \times \cdots \times g_{n}\right)
$$

with $g:=g_{1} \times \cdots \times g_{n}$ is smooth.

Proof Due to the chain rule, the pullback $g^{*}$ makes sense. Since $C^{\alpha}(L, N)$ is pre-canonical, $g^{*}$ will be smooth if $\left(g^{*}\right)^{\wedge}:(f, \ell) \mapsto \operatorname{ev}\left(f, \operatorname{ev}\left(\left(g_{1} \times \cdots \times g_{n}\right), \ell\right)\right)$ is a $C^{\infty, \alpha}$-map. Again, this is a consequence of Lemma 4.3 (a).

The key point was the differentiability of the evaluation map together with a suitable chain rule. Thus, by essentially the same proof, one obtains from the chain rule [1, Lemma 3.16] the following statement whose proof we omit.

Proposition 4.9 Assume that all the manifolds of mappings occurring in the following are endowed with pre-canonical manifold structures. Further, we let $\beta=\left(\beta_{1}, \ldots, \beta_{n}\right) \in\left(\mathbb{N}_{0} \cup\right.$ $\{\infty\})^{n}$ such that for multiindices $\alpha^{i} \in\left(\mathbb{N}_{0} \cup\{\infty\}\right)^{m_{i}}, i \in\{1, \ldots, n\}$ we have $\beta_{i}=\left|\alpha^{i}\right|+\sigma_{i}$ for some $\sigma_{i} \in \mathbb{N}_{0} \cup\{\infty\}$. Let now $N=\prod_{1 \leq i \leq n} N_{i}$ and $M^{i}:=M_{1}^{i} \times \cdots \times M_{m_{i}}^{i}$ for certain locally compact manifolds $M_{j}^{i}$ with rough boundary (with $j \in\left\{1, \ldots, m_{i}\right\}$ ). Then, for $\sigma=\left(\sigma_{1}, \ldots, \sigma_{n}\right)$ and $\alpha=\left(\alpha^{1}, \ldots, \alpha^{n}\right)$, the composition map

$$
\begin{aligned}
C^{\beta}(N, L) \times \prod_{1 \leq i \leq n} C^{\alpha^{i}}\left(M^{i}, N_{i}\right) & \rightarrow C^{\alpha}\left(M^{1} \times \cdots \times M^{n}, L\right), \\
\left(f, g_{1}, \ldots, g_{n}\right) & \mapsto f \circ\left(g_{1} \times \cdots \times g_{n}\right)
\end{aligned}
$$

is a $C^{\infty, \sigma}$-map.

The above discussion shows that composition, pushforward, and pullback maps inherit differentiability and continuity properties. The following variant will be used in the construction process of canonical manifold structures.

Proposition 4.10 Let $K$ be a compact smooth manifold such that $C^{\alpha}(K, M)$ and $C^{\alpha}(K, N)$ admit canonical manifold structures. If $\Omega \subseteq K \times M$ is an open subset and $f: \Omega \rightarrow N$ is a $C^{|\alpha|+k}$-map, then

$$
\Omega^{\prime}:=\left\{\gamma \in C^{\alpha}(K, M): \operatorname{graph}(\gamma) \subseteq \Omega\right\}
$$


is an open subset of $C^{\alpha}(K, M)$ and

$$
f_{\star}: \Omega \rightarrow C^{\alpha}(K, N), \gamma \mapsto f \circ\left(\operatorname{id}_{K}, \gamma\right)
$$

is a $C^{k}$-map.

Proof By compactness of $K$, the compact-open topology on $C(K, M)$ coincides with the graph topology (see, e.g., [15, Proposition A.6.25]). Thus, $\{\gamma \in C(K, M): \operatorname{graph}(\gamma) \subseteq \Omega\}$ is open in $C(K, M)$. As a consequence, $\Omega^{\prime}$ is open in $C^{\alpha}(K, M)$. By Lemma 4.3 (a), the evaluation ev: $C^{\alpha}(K, M) \times K \rightarrow M$ is $C^{\infty, \alpha}$ and hence $C^{k, \alpha}$, whence also $C^{\alpha}(K, M) \times$ $K \rightarrow K \times M,(\gamma, x) \mapsto(x, \gamma(x))$ is $C^{k, \alpha}$. Since $f$ is $C^{|\alpha|+k}$, the chain rule [1, Lemma 3.16] shows that

$$
\left(f_{\star}\right)^{\wedge}: \Omega^{\prime} \times K \rightarrow N, \quad(\gamma, x) \mapsto f_{\star}(\gamma)(x)=f(x, \gamma(x))
$$

is $C^{k, \alpha}$. So $f_{\star}$ is $C^{k}$, as the manifold structure on $C^{\alpha}(K, N)$ is canonical.

For later use, we record several observations on stability of (pre-)canonical structures under pushforward by diffeomorphisms.

Lemma 4.11 Let $N_{1}$ and $N_{2}$ be smooth manifolds and $\alpha \in\left(\mathbb{N}_{0} \cup\{\infty\}\right)^{n}, \beta \in\left(\mathbb{N}_{0} \cup\{\infty\}\right)^{m}$.

(a) If $C^{\alpha}\left(M, N_{1}\right)$ and $C^{\alpha}\left(M, N_{2}\right)$ are endowed with (pre-)canonical manifold structures, then the smooth manifold structure on $C^{\alpha}\left(M, N_{1} \times N_{2}\right)$ which turns the bijection $C^{\alpha}\left(M, N_{1} \times N_{2}\right) \rightarrow C^{\alpha}\left(M, N_{1}\right) \times C^{\alpha}\left(M, N_{2}\right)$ sending a mapping to the pair of component functions into a $C^{\infty}$-diffeomorphism, is (pre-)canonical.

(b) If $\psi: N_{1} \rightarrow N_{2}$ is a $C^{\infty}$-diffeomorphism and $C^{\alpha}\left(M, N_{2}\right)$ is a (pre-)canonical manifold, then the smooth manifold structure on $C^{\alpha}\left(M, N_{1}\right)$ turning the bijection

$$
\psi_{*}: C^{\alpha}\left(M, N_{1}\right) \rightarrow C^{\alpha}\left(M, N_{2}\right), \quad f \mapsto \psi \circ f
$$

into a diffeomorphism is (pre-)canonical.

(c) Let $C^{\alpha}(M, N)$ be endowed with a pre-canonical manifold structure and assume that both $C^{\beta}\left(L, C^{\alpha}(M, N)\right)$ and $C^{\beta, \alpha}(L \times M, N)$ are smooth manifolds making the bijection

$$
\Phi: C^{\beta, \alpha}(L \times M, N) \rightarrow C^{\beta}\left(L, C^{\alpha}(M, N)\right), \quad f \mapsto f^{\vee}
$$

a $C^{\infty}$-diffeomorphism. Then, $C^{\beta}\left(L, C^{\alpha}(M, N)\right)$ is pre-canonical if and only if the manifold $C^{\beta, \alpha}(L \times M, N)$ is pre-canonical.

Proof Let $L=L_{1} \times \cdots \times L_{m}$ be a product of manifolds.

(a) A map $f=\left(f_{1}, f_{2}\right): L \rightarrow C^{\alpha}\left(M, N_{1}\right) \times C^{\alpha}\left(M, N_{2}\right)$ is $C^{\beta}$ if and only if $f_{1}$ and $f_{2}$ are $C^{\beta}$. As the manifold structures are (pre-)canonical, this holds if and only if $f_{i}^{\wedge}: L \times M \rightarrow M_{i}$ is $C^{\beta, \alpha}$ for $i \in\{1,2\}$. However, this holds if and only if $f^{\wedge}=$ $\left(f_{1}^{\wedge}, f_{2}^{\wedge}\right)$ is $C^{\beta, \alpha}$.

(b) A map $f: L \rightarrow C^{\alpha}\left(M, N_{1}\right)$ is $C^{\beta}$ if and only if $\psi_{*} \circ f$ is $C^{\beta}$. Since $C^{\alpha}\left(M, N_{2}\right)$ is pre-canonical, this is the case if and only if $\left(\psi_{*} \circ f\right)^{\wedge}=\psi \circ f^{\wedge}$ is $C^{\beta, \alpha}$. As $\psi$ is a smooth diffeomorphism we deduce from the chain rule that this is the case if and only if $f^{\wedge}$ is of class $C^{\beta, \alpha}$. Thus, $C^{\alpha}\left(M, N_{1}\right)$ is pre-canonical. If $C^{\alpha}\left(M, N_{2}\right)$ is even canonical, the $C^{\alpha}$ topology is transported by the diffeomorphism $\psi_{*}$ to the $C^{\alpha}$-topology on $C^{\alpha}\left(M, N_{1}\right)$. Hence, the manifold $C^{\alpha}\left(M, N_{1}\right)$ is also canonical in this case. 
(c) By construction, a map $f: K \rightarrow C^{\beta, \alpha}(L \times M, N)$ is of class $C^{\gamma}$ (for some multiindex $\gamma$ ) if and only if $\Phi \circ f=(f(\cdot))^{\vee}$ is $C^{\gamma}$ as a mapping to $C^{\beta}\left(L, C^{\alpha}(M, N)\right)$. As $C^{\alpha}(M, N)$ is pre-canonical, we observe that $(\Phi \circ f)^{\wedge}: K \times L \rightarrow C^{\alpha}(M, N)$ is $C^{\gamma, \beta}$ if and only if $\left((\Phi \circ f)^{\wedge}\right)^{\wedge}=f^{\wedge}: K \times L \times M \rightarrow N$ is a $C^{\gamma, \beta, \alpha}$-map. Hence, $C^{\beta, \alpha}(L \times M, N)$ is pre-canonical (i.e., $f$ is $C^{\gamma}$ if and only if $f^{\wedge}$ is $\left.C^{\gamma, \beta, \alpha}\right)$ if and only if $C^{\beta}\left(L, C^{\alpha}(M, N)\right.$ ) is pre-canonical.

Lemma 4.12 Fix $\alpha \in\left(\mathbb{N}_{0} \cup\{\infty\}\right)^{n}$ and a permutation $\sigma$ of $\{1, \ldots, n\}$. Denote by $\phi_{\sigma}: M_{1} \times$ $\cdots \times M_{n} \rightarrow Q:=M_{\sigma(1)} \times \cdots \times M_{\sigma(n)}$ the diffeomorphism taking $\left(x_{i}\right)_{i=1}^{n}$ to $\left(x_{\sigma(i)}\right)_{i=1}^{n}$.

(a) If $C^{\alpha \circ \sigma}(Q, N)$ and $C^{\alpha}(M, N)$ are smooth manifolds such that the bijection

$$
\phi_{\sigma}^{*}: C^{\alpha \circ \sigma}(Q, N) \rightarrow C^{\alpha}(M, N), \quad f \mapsto f \circ \phi_{\sigma}
$$

from 2.13 becomes a diffeomorphism, then $C^{\alpha}(M, N)$ is (pre-)canonical if and only if $C^{\alpha \circ \sigma}(Q, N)$ is (pre-) canonical.

(b) If $C^{\alpha}(M, N)$ and $C^{\alpha \circ \sigma}(Q, N)$ are endowed with pre-canonical manifold structures, then $\phi_{\sigma}^{*}$ is a $C^{\infty}$-diffeomorphism.

(c) If $\psi_{i}: L_{i} \rightarrow M_{i}$ is a smooth diffeomorphism for every $i \in\{1, \ldots, n\}$ and $C^{\alpha}(M, N)$ is (pre-)canonical, then the smooth manifold structure on $C^{\alpha}(L, N)$ turning the bijection

$$
\left(\psi_{1} \times \cdots \times \psi_{n}\right)^{*}: C^{\alpha}(M, N) \rightarrow C^{\alpha}(L, N)
$$

into a diffeomorphism is (pre-)canonical.

Proof (a) Assume that $C^{\alpha}(M, N)$ is (pre-)canonical. Then, $f: K \rightarrow C^{\alpha \circ \sigma}(Q, N)$ is $C^{\beta}$ if and only if $\phi_{\sigma}^{*} \circ f$ is so. Now we deduce from $C^{\alpha}(M, N)$ being pre-canonical that this is equivalent to $\left(\phi_{\sigma}^{*} \circ f\right)^{\wedge}=f^{\wedge} \circ\left(\operatorname{id}_{K} \times \phi_{\sigma}\right): K \times M \rightarrow N$ being a $C^{\beta, \alpha}$-map. Exploiting the Theorem of Schwarz [1, Proposition 3.5], this is equivalent to $f^{\wedge}$ being $C^{\beta, \alpha \circ \sigma}$. Thus, $C^{\alpha \circ \sigma}(Q, N)$ is pre-canonical. The converse assertion for $C^{\alpha \circ \sigma}(M, N)$ follows verbatim by replacing $\phi_{\sigma}$ with its inverse. Note that if one of the manifolds is even canonical, it follows directly from the definition of the $C^{\alpha}$-topology, Definition 3.4, that reordering the factors induces a homeomorphism of the $C^{\alpha}$ - and $C^{\alpha \circ \sigma}$-topology. Hence, we see that one of the manifolds is canonical if and only if the other is so.

(b) Note that the inverse of $\phi_{\sigma}^{*}$ is $\left(\phi_{\sigma}^{-1}\right)^{*}$ whence the situation is symmetric and it suffices to prove that $\phi_{\sigma}^{*}$ (and by an analogous argument also its inverse) is smooth. As $C^{\alpha}(M, N)$ is pre-canonical, smoothness of $\phi_{\sigma}^{*}$ is equivalent to $\left(\phi_{\sigma}^{*}\right)^{\wedge}: C^{\alpha \circ \sigma}(Q, N) \times M \rightarrow$ $N,(f, m) \mapsto \operatorname{ev}\left(f, \phi_{\sigma}(m)\right)$ being a $C^{\infty, \alpha}$-mapping. This follows from Lemma $4.3(\mathrm{a})$, the chain rule, and Lemma 2.17.

(c) Replacing $\phi_{\sigma}$ with $\psi_{1} \times \cdots \times \psi_{n}$, the argument is analogous to (b). If $C^{\alpha}(M, N)$ is canonical, then the $C^{\alpha}$-topology pulls back to the $C^{\alpha}$-topology under the diffeomorphism, by Lemma 3.5.

An exponential law is available for pre-canonical smooth manifold structures.

Proposition 4.13 Let $L_{1}, \ldots, L_{m}$ and $N$ be smooth manifolds with rough boundary, and $M_{1}, \ldots, M_{n}$ be locally compact smooth manifolds with rough boundary. Assume that $C^{\alpha}(M, N)$ is endowed with a pre-canonical smooth manifold structure and also $C^{\beta}\left(L, C^{\alpha}(M, N)\right.$ and $C^{\beta, \alpha}(L \times M, N)$ are endowed with pre-canonical smooth manifold structures. Then, the bijection

$$
\Phi: C^{\beta, \alpha}(L \times M, N) \rightarrow C^{\beta}\left(L, C^{\alpha}(M, N)\right)
$$

from (1) is a $C^{\infty}$-diffeomorphism. 
Proof If we give $C^{\beta}\left(L, C^{\alpha}(M, N)\right)$ the smooth manifold structure making $\Phi$ a $C^{\infty}$. diffeomorphism, then this structure is pre-canonical by Lemma 4.11 (c). It therefore coincides with the given pre-canonical smooth manifold structure thereon, up to the choice of modeling spaces (Lemma $4.3(\mathrm{~b})$ ).

There is a natural identification of tangent vectors for pre-canonical manifolds, in good cases. If $C^{\alpha}(M, N)$ is pre-canonical, an element $v \in T_{f} C^{\alpha}(M, N)$ corresponds to an equivalence class of curves $\gamma_{v}: I \rightarrow C^{\alpha}(M, N)$ on some open interval $I$ around 0 such that $\gamma_{v}(0)=$ $f$ and $\dot{\gamma}_{v}(0)=v$. As $C^{\alpha}(M, N)$ is pre-canonical, the map $\gamma_{v}^{\wedge}: I \times M \rightarrow N$ is $C^{1, \alpha}$. Hence, $T \varepsilon_{m}(v)=T \varepsilon_{m}\left(\dot{\gamma}_{v}(0)\right) \in T N$ is $C^{\alpha}$ in $m \in M$, where we use the point evaluation $\varepsilon_{m}: C^{\alpha}(M, N) \rightarrow N, f \mapsto f(m)$ at $m$. We thus obtain a map

$$
\Psi: T C^{\alpha}(M, N) \rightarrow C^{\alpha}(M, T N), v \mapsto\left(m \mapsto T \varepsilon_{m}(v)\right) .
$$

Under additional assumptions, one can show that $\Psi$ is a diffeomorphism, allowing tangent vectors $v \in T C^{\alpha}(M, N)$ to be identified with $\Psi(v)$. We will encounter a setting in which this statement becomes true in the next section (see Theorem 5.14).

\section{Constructions for compact domains}

We now construct and study manifolds of $C^{\alpha}$-mappings on compact domains. The results of this section subsume Theorem 1.1. They generalize constructions for $C^{k, \ell}$-functions in [4, Appendix A].

5.1 Let $N$ be a smooth manifold, $\alpha \in\left(\mathbb{N}_{0} \cup\{\infty\}\right)^{n}$ and $M=M_{1} \times \cdots \times M_{n}$ be a locally compact smooth manifold with rough boundary. If $\pi: E \rightarrow N$ is a smooth vector bundle over $N$ and $f: M \rightarrow N$ is a $C^{\alpha}$-map, then we define

$$
\Gamma_{f}:=\left\{\tau \in C^{\alpha}(M, E): \pi \circ \tau=f\right\}
$$

with the topology induced by $C^{\alpha}(M, E)$. Pointwise operations turn $\Gamma_{f}$ into a vector space. Let us prove that $\Gamma_{f}$ is a locally convex space. To this end, we cover $N$ with open sets $\left(U_{i}\right)_{i \in I}$ on which the restriction $\left.E\right|_{U_{i}} \cong U_{i} \times E_{i}$ (with $E_{i}$ a suitable locally convex space) is trivial. Combining continuity of $f$ and local compactness of $M$ we can find families $\mathcal{K}_{j}$ of full compact submanifolds of $M_{j}$ with the following properties: The interiors of the sets in $\mathcal{K}_{j}$ cover $M_{j}$. There is a set $\mathcal{K} \subseteq \prod_{1 \leq j \leq n} \mathcal{K}_{j}$ such that for every $K=K_{1} \times \cdots \times K_{n} \in \mathcal{K}$ we have $f(K) \subseteq U_{i_{K}}$ for some $i_{K} \in I$ and the interiors of the submanifolds in $\mathcal{K}$ cover $M$. Hence, we deduce from Lemma 3.7 that the map

$$
\Psi: C^{\alpha}(M, E) \rightarrow \prod_{K \in \mathcal{K}} C^{\alpha}(K, E), \sigma \mapsto\left(\left.\sigma\right|_{K}\right)_{K \in \mathcal{K}}
$$

is a topological embedding. Now by construction $\Gamma_{f}$ is contained in the open subset $\left\{G \in C^{\alpha}(M, E) \mid G(K) \subseteq \pi^{-1}\left(U_{i_{K}}\right), \forall K \in \mathcal{K}\right\}$. Restricting $\Psi$ to this subset we obtain a topological embedding

$$
e: \Gamma_{f} \rightarrow \prod_{K \in \mathcal{K}} C^{\alpha}\left(K, \pi^{-1}\left(U_{i_{K}}\right)\right) \cong \prod_{K \in \mathcal{K}} C^{\alpha}\left(K, U_{i_{K}}\right) \times C^{\alpha}\left(K, E_{i_{K}}\right),
$$

where the identification exploits Lemma 3.11 and the fact that pushforwards with smooth diffeomorphisms induce homeomorphisms of the $C^{\alpha}$-topology (see Lemma 3.5). The image of $e$ are precisely the mappings which coincide on the intersections of the compact sets $K$ (see 
(10) and the explanations there). Hence, we can exploit that point evaluations are continuous on $C^{\alpha}\left(K, E_{i_{K}}\right)$ by [2, Proposition 3.17] to see that the image of $e$ is a closed vector subspace of $\prod_{K \in \mathcal{K}}\left\{\left.f\right|_{K}\right\} \times C^{\alpha}\left(K, E_{i_{K}}\right)$. As the space on the right-hand side is locally convex, we deduce that the co-restriction of $e$ onto its image is an isomorphism of locally convex spaces. Thus, $\Gamma_{f}$ is a locally convex topological vector space.

We will sometimes write $\Gamma_{f}(E)$ instead of $\Gamma_{f}$ to emphasize the dependence on the vector bundle $E$.

The previous setup allows an essential exponential law to be deduced.

Lemma 5.2 In the situation of 5.1 , let $\beta \in\left(\mathbb{N}_{0} \cup\{\infty\}\right)^{m}$ and $g: L \rightarrow \Gamma_{f}$ be a map, where $L_{1}, \ldots, L_{m}$ are smooth manifolds with rough boundary and $L:=L_{1} \times \cdots \times L_{m}$. Then, $g$ is $C^{\beta}$ if and only if

$$
g^{\wedge}: L \times M \rightarrow E, \quad(x, y) \mapsto g(x)(y)
$$

is a $C^{\beta, \alpha}$-map.

Proof With the notation as in 5.1 we identify $\Gamma_{f}$ via $e$ with a closed subspace of the locally convex space $\prod_{K \in \mathcal{K}} C^{\alpha}\left(K, E_{i_{K}}\right)$ (the identification will be suppressed in the notation). Thus, Lemma 2.14 (a) implies that the map $g$ is $C^{\beta}$ if and only if the components $g_{K}: L \rightarrow$ $C^{\alpha}\left(K, E_{i_{K}}\right)$ are $C^{\beta}$-maps. By the exponential law [1, Theorem 4.4], the latter holds if and only if the mappings

$$
\left(g_{K}\right)^{\wedge}: L \times K \rightarrow E_{i_{K}}, \quad(x, y) \mapsto g(x)(y)
$$

are of class $C^{\beta, \alpha}$. Since the interiors of sets $K \in \mathcal{K}$ cover $M$, we deduce that this is the case if and only if $g^{\wedge}$ is of class $C^{\beta, \alpha}$.

Remark 5.3 If all fibers of $E$ are Fréchet spaces and $K$ is $\sigma$-compact and locally compact, then $\Gamma_{F}$ is a Fréchet space; if all fibers of $E$ are Banach spaces, $K$ is compact, and $|\alpha|<\infty$, then $\Gamma_{f}$ is a Banach space. To see this, note that we can choose the family $\mathcal{K}$ in 5.1 countable (resp., finite). Suppressing again the identification,

$$
\psi: \Gamma_{f} \rightarrow \prod_{j \in J} C^{\alpha}\left(K_{j}, F_{j}\right), \quad \tau \mapsto\left(\left.\tau\right|_{K_{j}}\right)_{j \in J}
$$

is linear and a topological embedding with closed image. If all $F_{j}$ are Fréchet spaces, so is each $C^{\alpha}\left(K_{j}, F_{j}\right)$ (cf., e.g., [15]) and hence also $\Gamma_{f}$. If all $F_{j}$ are Banach spaces and $|\alpha|$ as well as $J$ is finite, then each $C^{\alpha}\left(K_{j}, F_{j}\right)$ is a Banach space (cf. loc. cit.) and hence also $\Gamma_{f}$.

Observe that the exponential law for $\Gamma_{f}$ gives this space the defining property of a precanonical manifold (and the only reason we do not call it pre-canonical is that it is only a subset of $\left.C^{\alpha}(M, E)\right)$. In particular, the proof of Lemma 4.3 (a) carries over and yields:

Lemma 5.4 In the situation of 5.1, the evaluation map

$$
\mathrm{ev}: \Gamma_{f} \times M \rightarrow E, \quad(\tau, x) \mapsto \tau(x)
$$

is $C^{\infty, \alpha}$.

Lemma 5.5 Let $\pi_{1}: E_{1} \rightarrow N$ and $\pi_{2}: E_{2} \rightarrow N$ be smooth vector bundles over a smooth manifold $N$. Let $\alpha \in\left(\mathbb{N}_{0} \cup\{\infty\}\right)^{m}$ and $f: M \rightarrow N$ be a $C^{\alpha}$-map on a product $M=$ $M_{1} \times \cdots \times M_{n}$ of smooth manifolds with rough boundary. Then, the following holds: 
(a) If $\psi: E_{1} \rightarrow E_{2}$ is a mapping of smooth vector bundles over $\mathrm{id}_{M}$, then $\psi \circ \tau \in \Gamma_{f}\left(E_{2}\right)$ for each $\tau \in \Gamma_{f}\left(E_{1}\right)$ and

$$
\Gamma_{f}(\psi): \Gamma_{f}\left(E_{1}\right) \rightarrow \Gamma_{f}\left(E_{2}\right), \quad \tau \mapsto \psi \circ \tau
$$

is a continuous linear map.

(b) $\Gamma_{f}\left(E_{1} \oplus E_{2}\right)$ is canonically isomorphic to $\Gamma_{f}\left(E_{1}\right) \times \Gamma_{f}\left(E_{2}\right)$.

Proof (a) If $\tau \in \Gamma_{f}\left(E_{1}\right)$, then $\psi \circ \tau: M \rightarrow E_{2}$ is $C^{\alpha}$ by the chain rule and $\pi_{2} \circ \psi \circ \tau=$ $\pi_{1} \circ \tau=f$, whence $\psi \circ \tau \in \Gamma_{f}\left(E_{2}\right)$. Evaluating at points we see that the map $\Gamma_{f}(\psi)$ is linear; being a restriction of the continuous map $C^{\alpha}(M, \psi): C^{\alpha}\left(M, E_{1}\right) \rightarrow C^{\alpha}\left(M, E_{2}\right)$ (see Lemma 3.5), it is continuous.

(b) If $\rho_{j}: E_{1} \oplus E_{2} \rightarrow E_{j}$ is the map taking $\left(v_{1}, v_{2}\right) \in E_{1} \times E_{2}$ to $v_{j}$ for $j \in\{1,2\}$ and $\iota_{j}: E_{j} \rightarrow E_{1} \oplus E_{2}$ is the map taking $v_{j} \in E_{j}$ to $\left(v_{1}, 0\right)$ and $\left(0, v_{2}\right)$, respectively, then

$$
\left(\Gamma_{f}\left(\rho_{1}\right), \Gamma_{f}\left(\rho_{2}\right)\right): \Gamma_{f}\left(E_{1} \oplus E_{2}\right) \rightarrow \Gamma_{f}\left(E_{1}\right) \times \Gamma_{f}\left(E_{2}\right)
$$

is a continuous linear map which is a homeomorphism as it has the continuous map $(\sigma, \tau) \mapsto \Gamma_{f}\left(\iota_{1}\right)(\sigma)+\Gamma_{f}\left(\iota_{2}\right)(\tau)$ as its inverse.

\section{Construction of the canonical manifold structure}

Having constructed spaces of $C^{\alpha}$-sections as model spaces, we are now in a position to construct the canonical manifold structure on $C^{\alpha}(K, M)$, assuming that $M$ is covered by local additions and $K$ is compact.

Definition 5.6 Let $M$ be a smooth manifold. A local addition is a smooth map

$$
\Sigma: U \rightarrow M
$$

defined on an open neighborhood $U \subseteq T M$ of the zero-section $0_{M}:=\left\{0_{p} \in T_{p} M: p \in M\right\}$ such that $\Sigma\left(0_{p}\right)=p$ for all $p \in M$,

$$
U^{\prime}:=\left\{\left(\pi_{T M}(v), \Sigma(v)\right): v \in U\right\}
$$

is open in $M \times M$ (where $\pi_{T M}: T M \rightarrow M$ is the bundle projection) and the map

$$
\theta:=\left(\pi_{T M}, \Sigma\right): U \rightarrow U^{\prime}
$$

is a $C^{\infty}$-diffeomorphism. If

$$
T_{0_{p}}\left(\left.\Sigma\right|_{T_{p} M}\right)=\operatorname{id}_{T_{p} M} \text { for all } p \in M,
$$

we say that the local addition $\Sigma$ is normalized.

Until Lemma 5.9, we fix the following setting, which allows a canonical manifold structure on $C^{\alpha}(K, M)$ to be constructed.

5.7 We consider a product $K=K_{1} \times K_{2} \times \cdots \times K_{n}$ of compact smooth manifolds with rough boundary, a smooth manifold $M$ which admits a local addition $\Sigma: T M \supseteq U \rightarrow M$, and $\alpha \in\left(\mathbb{N}_{0} \cup\{\infty\}\right)^{n}$. 


\subsection{Manifold structure on $C^{\alpha}(K, M)$ if $M$ admits a local addition}

For $f \in C^{\alpha}(K, M)$, let $\Gamma_{f}:=\left\{\tau \in C^{\alpha}(K, T M): \pi_{T M} \circ \tau=f\right\}$ be the locally convex space constructed in 5.1. Then,

$$
\begin{aligned}
& O_{f}:=\Gamma_{f} \cap C^{\alpha}(K, U) \text { is an open subset of } \Gamma_{f}, \\
& O_{f}^{\prime}:=\left\{g \in C^{\alpha}(K, M):(f, g)(K) \subseteq U^{\prime}\right\} \text { is an open subset of } C^{\alpha}(K, M), \text { and } \\
& \phi_{f}: O_{f} \rightarrow O_{f}^{\prime}, \quad \tau \mapsto \Sigma \circ \tau
\end{aligned}
$$

is a homeomorphism with inverse $g \mapsto \theta^{-1} \circ(f, g)$. By the preceding, if also $h \in C^{\alpha}(K, M)$, then $\psi:=\phi_{h}^{-1} \circ \phi_{f}$ has an open (possibly empty) domain $D \subseteq \Gamma_{f}$ and is a smooth map $D \rightarrow \Gamma_{h}$ by Lemma 5.2 , as $\psi^{\wedge}: D \times K \rightarrow T M$,

$$
(\tau, x) \mapsto\left(\phi_{h}^{-1} \circ \phi_{f}\right)(\tau)(x)=\theta^{-1}(h(x), \Sigma(\tau(x)))=\theta^{-1}(h(x), \Sigma(\varepsilon(\tau, x)))
$$

is a $C^{\infty, \alpha}$-map (exploiting that the evaluation map $\varepsilon: \Gamma_{f} \times K \rightarrow T M$ is $C^{\infty, \alpha}$, by Lemma 5.4). Hence, $C^{\alpha}(K, M)$ endowed with the $C^{\alpha}$-topology has a smooth manifold structure for which each of the maps $\phi_{f}^{-1}$ is a local chart.

We now prove that the manifold structure on $C^{\alpha}(K, M)$ is canonical. Together with Lemma 4.3 (b), this implies that the smooth manifold structure on $C^{\alpha}(K, M)$ constructed in 5.8 is independent of the choice of local addition.

Lemma 5.9 The manifold structure on $C^{\alpha}(K, M)$ constructed in 5.8 is canonical.

Proof We first show that the evaluation map ev: $C_{f}^{\alpha}(K, M) \times K \rightarrow M$ is $C^{\infty, \alpha}$. It suffices to show that $\operatorname{ev}\left(\phi_{f}(\tau), x\right)$ is $C^{\infty, \alpha}$ in $(\tau, x) \in O_{f} \times K$ for all $f \in C^{\alpha}(K, M)$. This follows from

$$
\operatorname{ev}\left(\phi_{f}(\tau), x\right)=\Sigma(\tau(x))=\Sigma(\varepsilon(\tau, x)),
$$

where $\varepsilon: \Gamma_{f} \times K \rightarrow T M,(\tau, x) \mapsto \tau(x)$ is $C^{\infty, \alpha}$ by Lemma 5.4. Now let $\beta \in\left(\mathbb{N}_{0} \cup\{\infty\}\right)^{m}$ and $h: N \rightarrow C^{\alpha}(K, M)$ be a map, where $N=N_{1} \times \cdots \times N_{n}$ is a product of smooth manifolds with rough boundary. If $h$ is $C^{\beta}$, then $h^{\wedge}=\mathrm{ev} \circ\left(h \times \mathrm{id}_{K}\right)$ is $C^{\beta, \alpha}$. Conversely, let $h^{\wedge}$ be a $C^{\beta, \alpha}$-map, then $h$ is continuous as a map to $C(K, M)$ with the compact-open topology (see [15, Proposition A.6.17]) and $h(x)=h^{\wedge}(x, \cdot) \in C^{\alpha}(K, M)$ for each $x \in N$. Given $x \in N$, let $f:=h(x)$. Then, $\psi_{f}: C(K, M) \rightarrow C(K, M) \times C(K, M) \cong C(K, M \times M)$, $g \mapsto(f, g)$ is a continuous map. Since $\psi_{f}(g)$ is $C^{\alpha}$ if and only if $g$ is $C^{\alpha}$, we see that

$$
\begin{aligned}
W & :=h^{-1}\left(O_{f}^{\prime}\right)=h^{-1}\left(\psi_{f}^{-1}\left(C^{\alpha}\left(K, U^{\prime}\right)\right)\right)=\left(\psi_{f} \circ h\right)^{-1}\left(C^{\alpha}\left(K, U^{\prime}\right)\right) \\
& =\left(\psi_{f} \circ h\right)^{-1}\left(C\left(K, U^{\prime}\right)\right)
\end{aligned}
$$

is an open $x$-neighborhood in $N$. As the map $\left(\left.\phi_{f}^{-1} \circ h\right|_{W}\right)^{\wedge}: W \times K \rightarrow T M$,

$$
(y, z) \mapsto\left(\left.\left(\phi_{f}\right)^{-1} \circ h\right|_{W}\right)^{\wedge}(y, z)=\left(\theta^{-1} \circ(f, h(y))\right)(z)=\theta^{-1}\left(f(z), h^{\wedge}(y, z)\right)
$$

is $C^{\beta, \alpha}$ by [1, Lemma 3.16], the map $\left.\phi_{f}^{-1} \circ h\right|_{W}: W \rightarrow \Gamma_{f}$ (and hence also $\left.h\right|_{W}$ ) is $C^{k}$, by Lemma 5.2.

Proposition 5.10 Let $K=K_{1} \times \cdots \times K_{n}$ be a product of compact smooth manifolds with rough boundary and $M$ be a manifold covered by local additions. For every $\alpha \in\left(\mathbb{N}_{0} \cup\{\infty\}\right)^{n}$, the set $C^{\alpha}(K, M)$ can be endowed with a canonical manifold structure. 
Proof Let $\left(M_{j}, \Sigma_{j}\right)_{j \in J}$ be an upward directed family of open submanifolds $M_{j}$ with local additions $\Sigma_{i}$ whose union coincides with $M$. As $K$ is compact, we observe that the sets $C^{\alpha}\left(K, M_{j}\right):=\left\{f \in C^{\alpha}(K, M) \mid f(K) \subseteq M_{j}\right\}$ are open in the $C^{\alpha}$-topology. Following Lemma 5.9, we can endow every $C^{\alpha}\left(K, M_{j}\right)$ with a canonical manifold structure. Now if $M_{j} \subseteq M_{\ell}$, Lemma 4.3 (c) implies that also the submanifold structure induced by the inclusion $C^{\alpha}\left(K, M_{j}\right) \subseteq C^{\alpha}\left(K, M_{\ell}\right)$ is canonical. Thus, uniqueness of canonical structures, Lemma 4.3(b), shows that the submanifold structure must coincide with the canonical structure constructed on $C^{\alpha}\left(K, M_{j}\right)$ via 5.8. As $C^{\alpha}(K, M)=\bigcup_{j \in J} C^{\alpha}\left(K, M_{j}\right)$ and each step of the ascending union is canonical, the same holds for the union.

\section{The tangent bundle of the manifold of mappings}

In the rest of this section, we identify the tangent bundle of $C^{\alpha}(K, M)$ as the manifold $C^{\alpha}(K, T M)$ (under the assumption that $K$ is compact and $M$ covered by local additions). To explain the idea, let us have a look at $C^{\alpha}(K, T M)$.

5.11 Consider a smooth manifold $M$ covered by local additions. Then also, $T M$ is covered by local additions, cf. [4, A.11] for the construction. Thus, for $K$ a compact manifold $C^{\alpha}(K, M)$ and $C^{\alpha}(K, T M)$ are canonical manifolds. If we denote by $\pi: T M \rightarrow M$ the bundle projection, Corollary 4.6 shows that the pushforward $\pi_{*}: C^{\alpha}(K, T M) \rightarrow C^{\alpha}(K, M)$ is smooth. The fibers of $\pi_{*}$ are the locally convex spaces $\pi_{*}^{-1}(f)=\Gamma_{f}$ from 5.1. We deduce that $\pi_{*}: C^{\alpha}(K, T M) \rightarrow C^{\alpha}(K, M)$ is a vector bundle (see Theorem 5.14 for a detailed proof).

We will first identify the fibers of the tangent bundle.

5.12 The tangent space $T_{f} C^{\alpha}(K, M)$ is given by equivalence classes $[t \mapsto c(t)]$ of $C^{1}$ curves $c:]-\varepsilon, \varepsilon\left[\rightarrow C^{\alpha}(K, M)\right.$ with $c(0)=f$, where the equivalence relation $c_{1} \sim c_{2}$ holds for two such curves if and only if $\dot{c}_{1}(0)=\dot{c}_{2}(0)$. Since the manifold structure is canonical (Lemma 5.10) we see that $c$ is $C^{1}$ if and only if the adjoint map $\left.c^{\wedge}:\right]-\varepsilon, \varepsilon[\times K \rightarrow N$ is a $C^{1, \alpha}$-map. The exponential law shows that the derivative of $c$ corresponds to the (partial) derivative of $c^{\wedge}$, i.e., the mapping $\Psi$ from (2) restricts to a bijection

$$
\begin{aligned}
\Psi_{f}: T_{\gamma} C^{\ell}(K, M) & \rightarrow \Gamma_{f}=\left\{h \in C^{\ell}(K, T M) \mid \pi \circ h=f\right\}, \\
{[c] \mapsto\left(k \mapsto\left[t \mapsto c^{\wedge}(t, k)\right]\right) . } & \mapsto \quad \text {. }
\end{aligned}
$$

We wish to glue the bijections on the fibers to identify the tangent manifold as the bundle from 5.11. To this end, we recall a fact from [4, Lemma A.14]:

5.13 If a manifold $M$ admits a local addition, it also admits a normalized local addition.

Hence, we may assume without loss of generality that the local additions in the following are normalized. Moreover, we will write $\varepsilon_{x}: C^{\alpha}(K, M) \rightarrow M$ for the point evaluation in $x \in K$. Then, the tangent bundle of $C^{\alpha}(K, M)$ can be described as follows.

Theorem 5.14 Let $K=K_{1} \times \cdots \times K_{n}$ be a product of compact smooth manifolds with rough boundary and $M$ be covered by local additions. Then,

$$
\left(\pi_{T M}\right)_{*}: C^{\ell}(K, T M) \rightarrow C^{\ell}(K, M)
$$

is a smooth vector bundle with fiber $\Gamma_{f}$ over $f \in C^{\ell}(K, M)$. For each $v \in T\left(C^{\ell}(K, M)\right)$, we have $\Psi(v):=\left(T \varepsilon_{x}(v)\right)_{x \in K} \in C^{\alpha}(K, T M)$ and the map (2), 


$$
\Psi: T C^{\alpha}(K, M) \rightarrow C^{\alpha}(K, T M), \quad v \mapsto \Psi(v)
$$

is an isomorphism of smooth vector bundles (over the identity).

If we wish to emphasize the dependence on $M$, we write $\Psi_{M}$ instead of $\Psi$.

Proof Since $M$ is covered by local additions, there is a family of open submanifolds (ordered by inclusion) $\left(M_{j}\right)_{j \in J}$ which admit local additions $\Sigma_{j}$. Now by compactness of $K$ the image of $f \in C^{\alpha}(K, M)$ is always contained in some $M_{j}$ and similarly for $\tau \in \Gamma_{f}$ we then have $\tau(K) \subseteq \pi^{-1}\left(M_{j}\right)=T M_{j}$, where $\pi:=\pi_{T M}$ is the bundle projection of $T M$. As the family $\left(M_{j}\right)_{j}$ of open manifolds exhausts $M$, we have $C^{\alpha}(K, M)=\bigcup_{j \in J} C^{\alpha}\left(K, M_{j}\right)$ and all of these subsets are open. Hence, it suffices to prove that $\Psi$ restricts to a bundle isomorphism for every $M_{j}$. In other words we may assume without loss of generality that $M$ admits a local addition $\Sigma$. Given $f \in C^{\alpha}(K, M)$, the map $\phi_{f}: O_{f} \rightarrow O_{f}^{\prime} \subseteq C^{\alpha}(K, M)$ is a $C^{\infty}$-diffeomorphism with $\phi_{f}(0)=f$, whence $T \phi_{f}(0, \cdot): \Gamma_{f} \rightarrow T_{f}\left(C^{\alpha}(K, M)\right)$ is an isomorphism of topological vector spaces. For $\tau \in \Gamma_{f}$, we have for $x \in K$

$$
\begin{aligned}
T \varepsilon_{x} T \phi_{f}(0, \tau) & =T \varepsilon_{x}([t \mapsto \Sigma \circ(t \tau)])=[t \mapsto \Sigma(t \tau(x))] \\
& =\left[\left.t \mapsto \Sigma\right|_{T_{f(x)} M}(t \tau(x))\right]=\left.T \Sigma\right|_{T_{f(x)} M}(\tau(x))=\tau(x),
\end{aligned}
$$

as $\Sigma$ is assumed normalized. Thus, $\Psi\left(T \phi_{f}(0, \tau)\right)=\tau \in \Gamma_{f} \subseteq C^{\alpha}(K, T M)$, whence $\Psi(v) \in \Gamma_{f} \subseteq C^{\alpha}(K, T M)$ for each $v \in T_{f}\left(C^{\alpha}(K, M)\right)$ and $\Psi$ takes $T_{f}\left(C^{\alpha}(K, M)\right)$ bijectively and linearly onto $\Gamma_{f}$. As the manifolds $T\left(C^{\alpha}(K, M)\right)$ and $C^{\alpha}(K, T M)$ are the disjoint union of the sets $T_{f}\left(C^{\alpha}(K, M)\right)$ and $\Gamma_{f}=\pi_{*}^{-1}(\{f\})$, respectively, we see that $\Psi$ is a bijection. If we can show that $\Psi$ is a $C^{\infty}$-diffeomorphism, $\pi_{*}: C^{\alpha}(K, T M) \rightarrow C^{\alpha}(K, M)$ will be a smooth vector bundle over $C^{\alpha}(K, M)$ (like $T\left(C^{\alpha}(K, M)\right)$ ). Finally, $\Psi$ will then be an isomorphism of smooth vector bundles over $\mathrm{id}_{M}$.

For the proof, we recall some results from the Appendix of [4]: Denote by $0: M \rightarrow T M$ the zero-section and by $0_{M}:=0(M)$ its image. Let now $\lambda_{p}: T_{p} M \rightarrow T M$ be the canonical inclusion and $\kappa: T^{2} M \rightarrow T^{2} M$ the canonical flip (given in charts by $(x, y, u, v) \mapsto(x, u, y, v)$ ). Then [4, Lemma A.20(b)] yields a natural isomorphism $\Theta: T M \oplus T M \rightarrow \pi_{T^{2} M}^{-1}\left(0_{M}\right) \subseteq$ $T^{2} M, \Theta(v, w)=\kappa\left(T \lambda_{\pi(v)}(v, w)\right)$. On the level of function spaces ${ }^{2} \Theta$ induces a diffeomorphism (cf. [4, Lemma A.20(e)])

$$
\Theta_{f}: O_{f} \rightarrow O_{0 \circ f}, \quad \gamma \mapsto \Theta \circ(0 \circ f, \gamma) .
$$

Here for $f \in C^{\alpha}(K, M)$ we have considered the composition $0 \circ f \in C^{\alpha}(K, T M)$. Then, the sets $S_{f}:=T \phi_{f}\left(O_{f} \times \Gamma_{f}\right)$ form an open cover of $T\left(C^{\alpha}(K, M)\right)$ for $f \in C^{\alpha}(K, M)$, whence the sets $\Psi\left(S_{f}\right)$ form a cover of $C^{\alpha}(K, T M)$ by sets which are open as $\Psi\left(S_{f}\right)=$ $\left(\phi_{0 \circ f} \circ \phi_{f}\right)\left(O_{f} \times \Gamma_{f}\right)=\phi_{0 \circ f}\left(O_{0 \circ f}\right)$. Hence, it suffices to prove that the bijective map $\Psi$ restricts to a $C^{\infty}$-diffeomorphism on these open sets. In other words it suffices to show that

$$
\Phi \circ T \phi_{f}=\phi_{0 \circ f} \circ \Theta_{f}
$$

for each $f \in C^{\ell}(K, M)$ (as all other mappings in the formula are smooth diffeomorphisms). Now

$$
T \phi_{f}(\sigma, \tau)=[t \mapsto \Sigma \circ(\sigma+t \tau)]
$$

2 While the results in [4] were only established for the case of $C^{k, \ell}$-mappings, they carry over (together with their proofs) without any change to the more general case of the $C^{\alpha}$-mappings considered here. 
for all $(\sigma, \tau) \in O_{f} \times \Gamma_{f}$, and thus we can rewrite $\Psi\left(T \phi_{f}(\sigma, \tau)\right)$ as

$$
\begin{aligned}
& ([t \mapsto \Sigma(\sigma(x)+t \tau(x))])_{x \in K}=\left(\left[t \mapsto\left(\Sigma \circ \lambda_{f(x)}\right)(\sigma(x)+t \tau(x))\right]\right)_{x \in K} \\
= & \left(T\left(\Sigma \circ \lambda_{f(x)}\right)(\sigma(x), \tau(x))\right)_{x \in K}=\left(\Sigma_{T M}\left(\left(\kappa \circ T \lambda_{f(x)}\right)(\sigma(x), \tau(x))\right)\right)_{x \in K} \\
= & \left(\left(\Sigma_{T M} \circ \Theta_{f}\right)(\sigma, \tau)(x)\right)_{x \in K}=\left(\phi_{0 \circ f} \circ \Theta_{f}\right)(\sigma, \tau) .
\end{aligned}
$$

Thus, the desired formula holds and shows that $\Psi$ is a $C^{\infty}$-diffeomorphism. This concludes the proof.

Remark 5.15 Assume that the local additions $\Sigma: U_{i} \rightarrow M_{i}$ covering $M$ are normalized. Then, the proof of Theorem 5.14 shows that

$$
\Psi \circ T \phi_{f}(0, \cdot): \Gamma_{f} \rightarrow C^{\alpha}(K, T M)
$$

is the inclusion map $\tau \mapsto \tau$, for each $f \in C^{\alpha}(K, M)$ (where $\phi_{f}$ is as in (5)).

Using canonical manifold structures, we have:

Corollary 5.16 Let $K=K_{1} \times \cdots \times K_{n}$ be a product of compact smooth manifolds with rough boundary, $\alpha \in\left(\mathbb{N}_{0} \cup\{\infty\}\right)^{n}$ and $g: M \rightarrow N$ be a $C^{|\alpha|+1}$-map between smooth manifolds $M$ and $N$ covered by local additions. Then, the tangent map of the $C^{1}$-map

$$
g_{*}: C^{\alpha}(K, M) \rightarrow C^{\alpha}(K, N), \quad f \mapsto g \circ f
$$

is given by $T\left(g_{*}\right)=\Psi_{N}^{-1} \circ(T g)_{*} \circ \Psi_{M}$. For each $f \in C^{\alpha}(K, M)$, we have $\Psi_{M}\left(T_{f}\left(C^{\alpha}(K, M)\right)\right)=\Gamma_{f}(T M), \Psi_{N}\left(T_{g \circ f}\left(C^{\alpha}(K, N)\right)\right)=\Gamma_{g \circ f}(T N)$ and $(T g)_{*}$ restricts to the map

$$
\Gamma_{f}(T M) \rightarrow \Gamma_{g \circ f}(T N), \quad \tau \mapsto T g \circ \tau
$$

which is continuous linear and corresponds to $T_{f}\left(g_{*}\right)$.

Moreover, the identification of the tangent bundle allows us to lift local additions (cf. [4, Remark A.17]).

Lemma 5.17 Let $K=K_{1} \times \cdots \times K_{n}$ be a product of compact smooth manifolds with rough boundary, $\alpha \in\left(\mathbb{N}_{0} \cup\{\infty\}\right)^{n}$ and $M$ a manifold covered by local additions. Then, the canonical manifold $C^{\alpha}(K, M)$ is covered by local additions.

Proof Consider first the case that $M$ admits a local addition $\Sigma: U \rightarrow M$ with $\theta=$ $\left(\pi_{T M}, \Sigma\right): U \rightarrow U^{\prime} \subseteq M \times M$ the associated diffeomorphism. Since also $T M$ admits a local addition, we have canonical manifold structures on $C^{\alpha}(K, T M)$ and $C^{\alpha}(K, M \times M) \cong$ $C^{\alpha}(K, M) \times C^{\alpha}(K, M)$. Now $K$ is compact, whence $C^{\alpha}(K, U) \subseteq C^{\alpha}(K, T M)$ is an open submanifold, whence canonical by Lemma 4.3 (c). In particular, $\Sigma_{*}: C^{\alpha}(K, U) \rightarrow$ $C^{\alpha}(K, M)$ and $\theta_{*}: C^{\alpha}(K, U) \rightarrow C^{\alpha}\left(K, U^{\prime}\right) \subseteq C^{\alpha}(K, M \times M)$ are smooth by Corollary 4.6. As also the inverse of $\theta$ is smooth, we deduce that $\theta_{*}$ is again a diffeomorphism mapping $C^{\alpha}(K, U)$ to $C^{\alpha}\left(K, U^{\prime}\right)$ and we can identify the latter manifold with an open subset of $C^{\alpha}(K, M) \times C^{\alpha}(K, M)$ containing the diagonal. Hence, we only need to verify that $0_{f} \in T_{f} C^{\alpha}(K, T M)$ is mapped to $f$. However, using the point evaluation $\varepsilon_{x}\left(\Sigma_{*}\left(0_{f}\right)\right)=\Sigma(0(f(x)))=f(x)$ (where 0 is again the zero-section of $T M$ ), we obtain the desired equality pointwise and thus also on the level of functions. This proves that $C^{\alpha}(K, M)$ admits a local addition if $M$ admits a local addition. 
If now $M$ is covered by open submanifolds $\left(M_{j}\right)_{j \in J}$ each admitting a local addition, it suffices to see that $C^{\alpha}\left(K, M_{j}\right)$ is an open submanifold of $C^{\alpha}(K, M)$ which admits a local addition by the above considerations. Thus, $C^{\alpha}(K, M)$ is covered by the open submanifolds $\left(C^{\alpha}\left(K, M_{j}\right)\right)_{j \in J}$ and as each of those admits a local addition, $C^{\alpha}(K, M)$ is covered by local additions.

Proposition 5.18 Let $K=K_{1} \times \cdots \times K_{m}$ and $L=L_{1} \times \cdots \times L_{n}$ be products of compact manifolds with rough boundary and $M$ be a manifold covered by local additions. Fix $\alpha \in\left(\mathbb{N}_{0} \cup\{\infty\}\right)^{n}, \beta \in\left(\mathbb{N}_{0} \cup\{\infty\}\right)^{m}$. Then, $C^{\beta, \alpha}(L \times K, M), C^{\alpha}(K, M)$ and $C^{\beta}\left(L, C^{\alpha}(K, M)\right)$ admit canonical manifold structures. Using these, the bijection $C^{\beta, \alpha}(L \times K, M) \rightarrow C^{\beta}\left(L, C^{\alpha}(K, M)\right)$ is a $C^{\infty}$-diffeomorphism.

Proof We apply Proposition 5.10 to obtain canonical manifold structures on $C^{\alpha}(K, M)$ and $C^{\beta, \alpha}(L \times K, M)$. By Lemma $5.17, C^{\alpha}(K, M)$ is covered by local additions. Hence, we may apply Proposition 5.10 again to obtain a canonical manifold structure on $C^{\beta}\left(L, C^{\alpha}(K, M)\right)$. By Proposition 4.13, the bijection $C^{\beta, \alpha}(L \times K, M) \rightarrow C^{\beta}\left(L, C^{\alpha}(K, M)\right)$ is a diffeomorphism.

\section{Lie groups of Lie group-valued mappings}

We now prove Theorem 1.3, starting with observations.

Lemma 6.1 Let $M_{1}, \ldots, M_{n}$ be locally compact smooth manifolds with rough boundary, $G$ be a Lie group, and $\alpha \in\left(\mathbb{N}_{0} \cup\{\infty\}\right)^{n}$. Setting $M:=M_{1} \times \cdots \times M_{n}$, the following holds:

(a) $C^{\alpha}(M, G)$ is a group.

(b) If a pre-canonical smooth manifold structure exists on $C^{\alpha}(M, G)$, then it makes $C^{\alpha}(M, G)$ a Lie group. Moreover, it turns the point evaluation $\varepsilon_{x}: C^{\alpha}(M, G) \rightarrow G$, $f \mapsto f(x)$ into a smooth group homomorphism for each $x \in M$.

Proof (a) The group inversion $\iota: G \rightarrow G$ is smooth, whence $\iota \circ f$ is $C^{\alpha}$ for all $f \in$ $C^{\alpha}(M, G)$ (by the chain rule [1, Lemma 3.16], applied in local charts). Let $\mu: G \times G \rightarrow$ $G$ be the smooth group multiplication and $f, g \in C^{\alpha}(M, G)$. Then, $(f, g): M \rightarrow G \times G$ is $C^{\alpha}$ by [1, Lemma 3.8]. By the chain rule, $f g=\mu \circ(f, g)$ is $C^{\alpha}$.

(b) The group inversion in $C^{\alpha}(M, G)$ is the map $C^{\alpha}(M, \iota)$ and hence smooth, by Corollary 4.6. Identifying $C^{\alpha}(M, G) \times C^{\alpha}(M, G)$ with $C^{\alpha}(M, G \times G)$ as a smooth manifold (as in Lemma 4.11 (a)), the group multiplication of $C^{\alpha}(M, G)$ is the map $C^{\alpha}(M, \mu)$ and hence smooth. The group multiplication in $C^{\alpha}(M, G)$ being pointwise, $\varepsilon_{x}$ is a homomorphism of groups for each $x \in M$. By Lemma 4.3(a), ev: $C^{\alpha}(M, G) \times M \rightarrow G$ is $C^{\infty, \alpha}$. Thus, $\varepsilon_{x}=\operatorname{ev}(\cdot, x)$ is smooth.

Another concept is useful, with notation as in 2.6.

Definition 6.2 Let $M_{1}, \ldots, M_{n}$ be locally compact smooth manifolds with rough boundary, $G$ be a Lie group, and $\alpha \in\left(\mathbb{N}_{0} \cup\{\infty\}\right)^{n}$. For $x \in M:=M_{1} \times \cdots \times M_{n}$, let $\varepsilon_{x}: C^{\alpha}(M, G) \rightarrow$ $G$ be the point evaluation. A smooth manifold structure on the set $C^{\alpha}\left(M_{1} \times \cdots \times M_{n}, G\right)$ making it a Lie group is said to be compatible with evaluations if $\varepsilon_{x}$ is smooth for each $x \in M$, we have $\phi(v):=\left(L\left(\varepsilon_{x}\right)(v)\right)_{x \in M} \in C^{\alpha}(M, L(G))$ for each $v \in L\left(C^{\alpha}(M, G)\right)$, and the Lie algebra homomorphism

$$
\phi: L\left(C^{\alpha}(M, G)\right) \rightarrow C^{\alpha}(M, L(G)), \quad v \mapsto \phi(v)
$$

so obtained is an isomorphism of topological vector spaces. 
Remark 6.3 In the case that $n=1$ and $\alpha=\infty$, compatibility with evaluations was introduced in [24, Proposition 1.9 and page 19] (in different words), assuming that $G$ is regular. Likewise, $G$ is assumed regular in [16, Proposition 3.1], where the case $n=1, \alpha \in \mathbb{N}_{0} \cup\{\infty\}$ is considered.

Lemma 6.4 Let $M_{1}, \ldots, M_{n}$ and $N_{1}, \ldots, N_{m}$ be locally compact smooth manifolds with rough boundary, $\alpha \in\left(\mathbb{N}_{0} \cup\{\infty\}\right)^{m}, \beta \in\left(\mathbb{N}_{0} \cup\{\infty\}\right)^{n}, M:=M_{1} \times \cdots \times M_{n}, N:=$ $N_{1} \times \cdots \times N_{m}$, and $G$ be a Lie group. Assume that $C^{\beta}(M, G)$ is endowed with a pre-canonical smooth manifold structure which is compatible with evaluations and that $C^{\alpha}\left(N, C^{\beta}(M, G)\right)$, whose definition uses the latter structure, is endowed with a pre-canonical smooth manifold structure which is compatible with evaluations. Endow $C^{\alpha, \beta}(N \times M, G)$ with the smooth manifold structure turning the bijection

$$
\Phi: C^{\alpha, \beta}(N \times M, G) \rightarrow C^{\alpha}\left(N, C^{\beta}(M, G)\right), \quad f \mapsto f^{\vee}
$$

into a $C^{\infty}$-diffeomorphism. Then, the preceding smooth manifold structure on $C^{\alpha, \beta}(N \times$ $M, G)$ is pre-canonical and compatible with evaluations.

Proof By Lemma 4.11 (c), the $C^{\infty}$-manifold structure on $C^{\alpha, \beta}(N \times M, G)$ is pre-canonical, whence the latter is a Lie group. The $C^{\infty}$-diffeomorphism $\Phi$ is a homomorphism of groups. Hence,

$$
L(\Phi): L\left(C^{\alpha, \beta}(N \times M, G)\right) \rightarrow L\left(C^{\alpha}\left(N, C^{\beta}(M, G)\right)\right)
$$

is an isomorphism of topological Lie algebras. Consider the point evaluations $\varepsilon_{x}$ : $C^{\alpha}\left(N, C^{\beta}(M, G)\right) \rightarrow C^{\beta}(M, G), \varepsilon_{(x, y)}: C^{\alpha, \beta}(N \times M, G) \rightarrow G$ and $\varepsilon_{y}: C^{\beta}(M, G) \rightarrow G$ for $x \in N, y \in M$. By hypothesis, we have isomorphisms of topological Lie algebras

$$
\Psi: L\left(C^{\beta}(M, G)\right) \rightarrow C^{\beta}(M, L(G)), w \mapsto\left(L\left(\varepsilon_{y}\right)(w)\right)_{y \in M}
$$

and $\Theta: L\left(C^{\alpha}\left(N, C^{\beta}(M, G)\right)\right) \rightarrow C^{\alpha}\left(N, L\left(C^{\beta}(M, G)\right)\right), v \mapsto\left(L\left(\varepsilon_{x}\right)(v)\right)_{x \in N}$. Then also,

$$
\Psi_{*}: C^{\alpha}\left(N, L\left(C^{\beta}(M, G)\right)\right) \rightarrow C^{\alpha}\left(N, C^{\beta}(M, L(G))\right), \quad f \mapsto \Psi \circ f
$$

is an isomorphism of topological Lie algebras and so is

$$
\Xi: C^{\alpha}\left(N, C^{\beta}(M, L(G)) \rightarrow C^{\alpha, \beta}(N \times M, L(G)), \quad f \mapsto f^{\wedge},\right.
$$

by the exponential law (Lemma 3.13). Hence,

$$
\phi:=\Xi \circ \Psi_{*} \circ \Theta \circ L(\Phi): L\left(C^{\alpha, \beta}(N \times M, G)\right) \rightarrow C^{\alpha, \beta}(M \times N, L(G))
$$

is an isomorphism of topological Lie algebras. Regard $v \in L\left(C^{\alpha, \beta}(N \times M, G)\right)$ as a geometric tangent vector $[\gamma]$ for a smooth curve $\gamma:]-\varepsilon, \varepsilon\left[\rightarrow C^{\alpha, \beta}(N \times M, G)\right.$ with $\gamma(0)=e$. Then, $L(\Phi)(v)=[\Phi \circ \gamma]$ and $\Theta(L(\Phi)(v))=\left(\left[\varepsilon_{x} \circ \Phi \circ \gamma\right]\right)_{x \in N}=: g$. Thus,

$$
\begin{aligned}
\phi(v)(x, y) & =\Psi_{*}(g)(x)(y)=(\Psi \circ g)(x)(y)=\Psi\left(\left[\varepsilon_{x} \circ \Phi \circ \gamma\right]\right)(y) \\
& =L\left(\varepsilon_{y}\right)\left(\left[\varepsilon_{x} \circ \Phi \circ \gamma\right]\right)=\left[\varepsilon_{x} \circ \varepsilon_{y} \circ \Phi \circ \gamma\right]=\left[t \mapsto \varepsilon_{x}\left(\varepsilon_{y}(\Phi(\gamma(t)))\right)\right] \\
& =[t \mapsto \gamma(t)(x, y)]=L\left(\varepsilon_{(x, y)}\right)([\gamma])=L\left(\varepsilon_{(x, y)}\right)(v) .
\end{aligned}
$$

We deduce that $\left(L\left(\varepsilon_{(x, y)}\right)(v)\right)_{(x, y) \in N \times M}=\phi(v) \in C^{\alpha, \beta}(N \times M, L(G))$. Since $\phi$ is an isomorphism of topological Lie algebras, the Lie group structure on $C^{\alpha, \beta}(N \times M, G)$ is compatible with evaluations. 
Lemma 6.5 Let $M_{1}, \ldots, M_{n}$ be locally compact smooth manifolds with rough boundary, $M:=M_{1} \times \cdots \times M_{n}, \alpha \in\left(\mathbb{N}_{0} \cup\{\infty\}\right)^{n}$, and $G$ be a Lie group. Assume that $C^{\alpha}(M, G)$ is endowed with a pre-canonical smooth manifold structure which is compatible with evaluations. If the Lie group $G$ is $C^{r}$-regular for some $r \in \mathbb{N}_{0} \cup\{\infty\}$, then also the Lie group $C^{\alpha}(M, G)$ is $C^{r}$-regular.

Proof Consider the smooth evolution map Evol: $C^{r}([0,1], \mathfrak{g}) \rightarrow C^{r+1}([0,1], G)$, where $\mathfrak{g}:=L(G)$. For $x \in M$, let $\varepsilon_{x}: C^{\alpha}(M, G) \rightarrow G, f \mapsto f(x)$ be evaluation at $x$. By hypothesis, $\phi: L\left(C^{\alpha}(M, G)\right) \rightarrow C^{\alpha}(M, \mathfrak{g}), v \mapsto\left(L\left(\varepsilon_{x}\right)(v)\right)_{x \in M}$ is an isomorphism of topological Lie algebras. Then also,

$$
\phi_{*}: C^{r}\left([0,1], L\left(C^{\alpha}(M, G)\right)\right) \rightarrow C^{r}\left([0,1], C^{\alpha}(M, \mathfrak{g})\right), f \mapsto \phi \circ f
$$

is an isomorphism of topological Lie algebras. By Example 4.1, the smooth manifold structures on all of the locally convex spaces $C^{r}\left([0,1], C^{\alpha}(M, \mathfrak{g})\right)$,

$$
C^{r, \alpha}([0,1] \times M, \mathfrak{g}), \quad C^{\alpha, r}(M \times[0,1], \mathfrak{g}), \quad \text { and } C^{\alpha}\left(M, C^{r}([0,1], \mathfrak{g})\right)
$$

are canonical. By Lemma 3.13, the Lie algebra homomorphism

$$
\psi: C^{r}\left([0,1], C^{\alpha}(M, \mathfrak{g})\right) \rightarrow C^{r, \alpha}([0,1] \times M, \mathfrak{g}), \quad f \mapsto f^{\wedge}
$$

is an isomorphism of topological Lie algebras. Flipping the factors $[0,1]$ and $M$ (with Lemma 4.12 (b)) and using the exponential law again, we obtain an isomorphism of topological Lie algebras

$$
\theta: C^{r, \alpha}([0,1] \times M, \mathfrak{g}) \rightarrow C^{\alpha}\left(M, C^{r}([0,1], \mathfrak{g})\right)
$$

determined by $\theta(f)(x)(t)=f(t, x)$. By Theorem 1.1, $C^{r+1}\left([0,1], C^{\alpha}(M, G)\right)$ has a canonical smooth manifold structure. Using Lemmas 4.11 (c), 4.12 (a), and 4.11 (c) in turn, we can give $C^{\alpha}\left(M, C^{r+1}([0,1], G)\right)$ a pre-canonical smooth manifold structure making the map

$$
\beta: C^{\alpha}\left(M, C^{r+1}([0,1], G)\right) \rightarrow C^{r+1}\left([0,1], C^{\alpha}(M, G)\right)
$$

determined by $\beta(f)(t)(x)=f(x)(t)$ a $C^{\infty}$-diffeomorphism. The structures being precanonical,

$$
\operatorname{Evol}_{*}: C^{\alpha}\left(M, C^{r}([0,1], \mathfrak{g})\right) \rightarrow C^{\alpha}\left(M, C^{r+1}([0,1], G)\right), \quad f \mapsto \text { Evol } \circ f
$$

is smooth. Hence also, $\mathcal{E}:=\beta \circ \mathrm{Evol}_{*} \circ \theta \circ \psi \circ \phi_{*}$ is smooth as a map

$$
C^{r}\left([0,1], L\left(C^{\alpha}(M, G)\right)\right) \rightarrow C^{r+1}\left([0,1], C^{\alpha}(M, G)\right) .
$$

It remains to show that $\mathcal{E}$ is the evolution map of $C^{\alpha}(M, G)$. As the $L\left(\varepsilon_{x}\right)$ separate points on $\mathfrak{h}:=L\left(C^{\alpha}(M, G)\right)$ for $x \in M$, it suffices to show that $\varepsilon_{x} \circ \mathcal{E}(\gamma)=\operatorname{Evol}\left(L\left(\varepsilon_{x}\right) \circ \gamma\right)$ for all $\gamma \in C^{r}([0,1], \mathfrak{h})$ and $x \in M\left(\right.$ see $\left[12\right.$, Lemma 10.1]). Note that $(\phi \circ \gamma)(t)(x)=L\left(\varepsilon_{x}\right)(\gamma(t))$, whence

$$
((\psi \circ \theta)(\phi \circ \gamma))(x)(t)=L\left(\varepsilon_{x}\right)(\gamma(t))
$$

and $\left(\operatorname{Evol}_{*}((\psi \circ \theta)(\phi \circ \gamma))\right)(x)=\operatorname{Evol}(((\psi \circ \theta)(\phi \circ \gamma))(x))=\operatorname{Evol}\left(L\left(\varepsilon_{x}\right) \circ \gamma\right)$. So $\left(\varepsilon_{x} \circ \mathcal{E}(\gamma)\right)(t)=\left(\operatorname{Evol}_{*} \circ \theta \circ \psi \circ \phi_{*}\right)(\gamma)(x)(t)=\operatorname{Evol}\left(L\left(\varepsilon_{x}\right) \circ \gamma\right)(t)$.

We establish Theorem 1.3 in parallel with the first conclusion of the following proposition, starting with two basic cases:

Case 1: The manifolds $M_{1}, \ldots, M_{n}$ are compact;

Case 2: $M$ is 1-dimensional with finitely many connected components. 
Proposition 6.6 In Theorem 1.3, the Lie group structure on $C^{\alpha}(M, G)$ is compatible with evaluations, writing $M:=M_{1} \times \cdots \times M_{n}$. Moreover, there is a unique canonical pure smooth manifold structure on $C^{\alpha}(M, G)$ which is modeled on $C^{\alpha}(M, L(G))$.

The final assertion is clear: Starting with any canonical structure on $C^{\alpha}(M, G)$ and a chart $\phi: U_{\phi} \rightarrow V_{\phi} \rightarrow E_{\phi}$ around the constant map $e$, using left translations (which are $C^{\infty}$ diffeomorphisms) we can create charts around every $f \in C^{\alpha}(M, G)$ which are modeled on the given $E_{\phi}$. We can therefore select a subatlas making $C^{\alpha}(M, G)$ a pure smooth manifold. Since $E_{\phi}$ is isomorphic to $L\left(C^{\alpha}(M, G)\right)$, which is isomorphic to $E:=C^{\alpha}(M, L(G))$ as a locally convex space (by compatibility with evaluations), we can replace $E_{\phi}$ with $E$. The pure canonical structure modeled on $E$ is unique, since $\operatorname{id}_{C^{\alpha}(M, G)}$ is a $C^{\infty}$-diffeomorphism for any two canonical structures (cf. Lemma 4.3 (b)).

Lemma 6.7 Let $M_{1}, \ldots, M_{n}$ be compact smooth manifolds with rough boundary, $G$ be a Lie group and $\alpha \in\left(\mathbb{N}_{0} \cup\{\infty\}\right)^{n}$. Abbreviate $M:=M_{1} \times \cdots \times M_{n}$. Then, $C^{\alpha}(M, G)$ admits a canonical smooth manifold structure which is compatible with evaluations. If $G$ is $C^{r}$-regular for $r \in \mathbb{N}_{0} \cup\{\infty\}$, then so is $C^{\alpha}(M, G)$.

Proof By Theorem 1.1, $C^{\alpha}(M, G)$ admits a canonical smooth manifold structure. Let $\theta: M \rightarrow G$ be the constant map $x \mapsto e$. By Theorem 5.14, the diffeomorphism $\left(T \varepsilon_{x}\right)_{x \in M}$ maps $L\left(C^{\alpha}(M, G)\right)=T_{\theta}\left(C^{\alpha}(M, G)\right)$ onto

$$
\Gamma_{\theta}=\left\{\tau \in C^{\alpha}(M, T G): \pi_{T G} \circ \tau=\theta\right\}=C^{\alpha}(M, L(G)) .
$$

By Lemma 3.9, $C^{\alpha}(M, T G)$ induces on $C^{\alpha}(M, L(G))$ the compact-open $C^{\alpha}$-topology. Thus, the Lie group structure on $C^{\alpha}(M, G)$ is compatible with evaluations. For the last assertion, see Lemma 6.5.

Lemma 6.8 Let M be a 1-dimensional smooth manifold with rough boundary, such that $M$ has only finitely many connected components (which need not be $\sigma$-compact). Let $r \in \mathbb{N}_{0} \cup\{\infty\}$, $G$ be a $C^{r}$-regular Lie group, and $k \in \mathbb{N} \cup\{\infty\}$ such that $k \geq r+1$. Then, $C^{k}(M, G)$ admits a canonical smooth manifold structure which makes it a $C^{r}$-regular Lie group and is compatible with evaluations.

Proof We first assume that $M$ is connected. Let $\mathfrak{g}:=L(G)$ be the Lie algebra of $G$. If $N$ is a full submanifold of $M$, we write $\Omega_{C^{k-1}}^{1}(N, \mathfrak{g}) \subseteq C^{k-1}(T N, \mathfrak{g})$ for the locally convex space of $\mathfrak{g}$-valued 1-forms on $N$, of class $C^{k-1}$. Using the Maurer-Cartan form

$$
\kappa: T G \rightarrow \mathfrak{g}, \quad v \mapsto \pi_{T G}(v)^{-1} . v,
$$

a g-valued 1-form

$$
\delta_{N}(f):=\kappa \circ T f \in \Omega_{C^{k-1}}^{1}(N, \mathfrak{g})
$$

can be associated to each $f \in C^{k}(N, G)$, called its left logarithmic derivative. Fix $x_{0} \in M$. For every $\sigma$-compact, connected, full submanifold $N \subseteq M$ such that $x_{0} \in N$, there exists a $C^{\infty}$-diffeomorphism $\psi: I \rightarrow N$ for some non-degenerate interval $I \subseteq \mathbb{R}$, such that $0 \in I$ and $\psi(0)=x_{0}$. Then, the diagram

$$
\begin{array}{ccc}
C^{k}(N, G) & \stackrel{\delta_{N}}{\longrightarrow} & \Omega_{C^{k-1}}^{1}(N, \mathfrak{g}) \\
\psi^{*} \downarrow & & \downarrow \theta \\
C^{k}(I, G) & \stackrel{\delta^{\ell}}{\longrightarrow} & C^{k-1}(I, \mathfrak{g}),
\end{array}
$$


is commutative, where $\psi^{*}: C^{k}(N, G) \rightarrow C^{k}(I, G), f \mapsto f \circ \psi$ and the vertical map $\theta$ on the right-hand side, which takes $\omega$ to $\omega \circ \dot{\psi}$, are bijections. For each $\omega \in \Omega_{C^{k-1}}^{1}(N, \mathfrak{g})$, there is a unique $f \in C^{k}(N, G)$ such that $f\left(x_{0}\right)=e$ and $\delta_{N}(f)=\omega$ : In fact, Lemma 2.8 yields a unique $\eta \in C^{k}(I, G)$ with $\eta(0)=e$ and $\delta^{\ell}(\eta)=\theta(\omega)$; then $f:=\left(\psi^{*}\right)^{-1}(\eta)$ is as required. We set $\operatorname{Evol}_{N}(\omega):=f$.

If $\omega \in \Omega_{C^{k-1}}^{1}(M, \mathfrak{g})$, we have $\operatorname{Evol}_{L}\left(\left.\omega\right|_{T L}\right)=\left.\operatorname{Evol}_{N}\left(\left.\omega\right|_{T N}\right)\right|_{L}$ for all $\sigma$-compact, connected open submanifolds $N, L$ of $M$ such that $L \subseteq N$. As such submanifolds $N$ form a cover of $M$ which is directed under inclusion, we can define $f: M \rightarrow G$ piecewise via $f(x):=$ $\operatorname{Evol}_{N}\left(\left.\omega\right|_{T N}\right)(x)$ if $x \in N$ and obtain a well-defined $C^{k}$-map $f: M \rightarrow G$ such that $\delta_{M}(f)=$ $\omega$. Thus,

$$
\delta_{M}\left(C^{k}(M, G)\right)=\Omega_{C^{k-1}}^{1}(M, \mathfrak{g}),
$$

which is a submanifold of $\Omega_{C^{k-1}}^{1}(M, \mathfrak{g})$. Let $\mathcal{K}$ be the set of all connected, compact full submanifolds $K \subseteq M$ such that $x_{0} \in K$. By the preceding, $\delta_{K}\left(C^{k}(K, G)\right)=\Omega_{C^{k-1}}^{1}(K, \mathfrak{g})$, which is a submanifold of $\Omega_{C^{k-1}}^{1}(K, \mathfrak{g})$. Since

$$
M=\bigcup_{K \in \mathcal{K}} K^{o},
$$

[16, Theorem 3.5] provides a smooth manifold structure on $C^{k}(M, \mathfrak{g})$ which makes it a $C^{r}$-regular Lie group, is compatible with evaluations, and turns

$$
\psi: C^{k}(M, G) \rightarrow \Omega_{C^{k-1}}^{1}(M, \mathfrak{g}) \times G, f \mapsto\left(\delta_{M}(f), f\left(x_{0}\right)\right)
$$

into a $C^{\infty}$-diffeomorphism. It remains to show that the smooth manifold structure is canonical. To prove the latter, we first note that $\mathcal{K}$ is directed under inclusion. In fact, if $K_{1}, K_{2} \in \mathcal{K}$, then $K_{1} \cup K_{2}$ is contained in a $\sigma$-compact, connected open submanifold $N$ of $M$ (a union of chart domains diffeomorphic to convex subsets of $\mathbb{R}$, around finitely many points in the compact set $\left.K_{1} \cup K_{2}\right)$. Pick a $C^{\infty}$-diffeomorphism $\psi: I \rightarrow N$ as above. Then, $\psi^{-1}\left(K_{1}\right)$ and $\psi^{-1}\left(K_{2}\right)$ are compact intervals containing 0 , whence so is their union. Thus, $K_{1} \cup K_{2}$ is a connected, compact full submanifold of $N$ and hence of $M$.

For $K, L \in \mathcal{K}$ with $K \subseteq L$, let $r_{K, L}: \Omega_{C^{k-1}}^{1}(L, \mathfrak{g}) \rightarrow \Omega_{C^{k-1}}^{1}(K, \mathfrak{g})$ be the restriction map. As a consequence of Lemma 3.7 and (8),

$$
\Omega_{C^{k-1}}^{1}(M, \mathfrak{g})=\lim _{K \in \mathcal{K}} \Omega_{C^{k-1}}^{1}(K, \mathfrak{g})
$$

holds as a locally convex space, using the restriction maps $r_{K}: \Omega_{C^{k-1}}^{1}(M, \mathfrak{g}) \rightarrow \Omega_{C^{k-1}}^{1}(K, \mathfrak{g})$ as the limit maps. For $K \in \mathcal{K}$, let $\rho_{K}: C^{k}(M, G) \rightarrow C^{k}(K, G)$ be the restriction map; endow $C^{k}(K, G)$ with its canonical smooth manifold structure (as in Lemma 6.7), which is compatible with evaluations (the "ordinary" Lie group structure in [16]). Then,

$$
\psi_{K}: C^{k}(K, G) \rightarrow \Omega_{C^{k-1}}^{1}(K, \mathfrak{g}) \times G, \quad f \mapsto\left(\delta_{K}(f), f\left(x_{0}\right)\right)
$$

is a $C^{\infty}$-diffeomorphism (see [16, proof of Theorem 3.5]). Note that $\rho_{K}=\psi_{K}^{-1} \circ\left(r_{K} \times\right.$ $\left.\operatorname{id}_{G}\right) \circ \psi$ is smooth on $C^{k}(M, G)$, using the above Lie group structure making $\psi$ a $C^{\infty}$ diffeomorphism. Let $\alpha \in\left(\mathbb{N}_{0} \cup\{\infty\}\right)^{m}, L_{1}, \ldots, L_{m}$ be smooth manifolds with rough boundary, $L:=L_{1} \times \cdots \times L_{m}$ and $f: L \rightarrow C^{k}(M, G)$ be a map. If $f$ is $C^{\alpha}$, then also $\rho_{K} \circ f$ is $C^{\alpha}$. Since $C^{k}(K, G)$ is canonical, the map

$$
\left.f^{\wedge}\right|_{L \times K}=\left(\rho_{j} \circ f\right)^{\wedge}: L \times K \rightarrow G
$$


is $C^{\alpha, k}$. Using (8), we deduce that $f^{\wedge}$ is $C^{\alpha, k}$. If, conversely, $f^{\wedge}$ is $C^{\alpha, k}$, then $\left(\rho_{K} \circ f\right)^{\wedge}=$ $\left.f^{\wedge}\right|_{L \times K}$ is $C^{\alpha, k}$. The smooth manifold structure on $C^{k}(K, G)$ being canonical, we deduce that $\rho_{K} \circ f$ is $C^{\alpha}$. The hypotheses of Lemma 2.15 being satisfied with $A:=\mathcal{K}, C^{k}(M, G)$ in place of $M, M_{K}:=C^{k}(K, G), F:=\Omega_{C^{k-1}}^{1}(M, \mathfrak{g}), F_{K}:=\Omega_{C^{k-1}}^{1}(K, \mathfrak{g})$, and $N:=G$, we see that $f$ is $C^{\alpha}$. The smooth manifold structure on $C^{k}(M, G)$ is therefore pre-canonical. The topology on the projective limit $\Omega_{C^{k-1}}^{1}(M, \mathfrak{g})$ is initial with respect to the limit maps $r_{K}$, whence the topology on $\Omega_{C^{k-1}}^{1}(M, \mathfrak{g}) \times G$ is initial with respect to the maps $r_{K} \times \mathrm{id}_{G}$. Since $\psi$ is a homeomorphism, we deduce that the topology $\mathcal{O}$ on the Lie group $C^{k}(M, G)$ is initial with respect to the maps $\left(r_{K} \times \mathrm{id}_{G}\right) \circ \psi=\psi_{K} \circ \rho_{K}$. Since $\psi_{K}$ is a homeomorphism, $\mathcal{O}$ is initial just as well with respect to the family $\left(\rho_{K}\right)_{K \in \mathcal{K}}$. But also the compact-open $C^{k}$-topology $\mathcal{T}$ on $C^{k}(M, G)$ is initial with respect to this family of maps (see Lemma 3.7), whence $\mathcal{O}=\mathcal{T}$ and $C^{k}(M, G)$ is canonical.

If $M$ has finitely many components $M_{1}, \ldots, M_{n}$, we give $C^{k}(M, G)$ the smooth manifold structure turning the bijection

$$
\rho: C^{k}(M, G) \rightarrow \prod_{j=1}^{n} C^{k}\left(M_{j}, G\right), \quad f \mapsto\left(\left.f\right|_{M_{j}}\right)_{j=1}^{n}
$$

into a $C^{\infty}$-diffeomorphism. Let $\rho_{j}$ be its $j$ th component. Since $\rho$ is a homeomorphism for the compact-open $C^{k}$-topologies (cf. Lemma 3.7) and an isomorphism of groups, the preceding smooth manifold structure makes $C^{k}(M, G)$ a Lie group and is compatible with the compact-open $C^{k}$-topology. As each of the Lie groups $C^{k}\left(M_{j}, G\right)$ is $C^{r}$-regular, also their direct product (and thus $\left.C^{k}(M, G)\right)$ is $C^{r}$-regular. Since $\rho=\left(\rho_{j}\right)_{j=1}^{n}$ is an isomorphism of Lie groups,

$$
\left(L\left(\rho_{1}\right), \ldots L\left(\rho_{n}\right)\right): L\left(C^{k}(M, G)\right) \rightarrow L\left(C^{k}\left(M_{1}, G\right)\right) \times \cdots \times L\left(C^{k}\left(M_{n}, G\right)\right)
$$

is an isomorphism of topological Lie algebras. For $x \in M_{j}$, the point evaluation $\varepsilon_{x}: C^{k}(M, G) \rightarrow G$ is smooth, as the point evaluation $\bar{\varepsilon}_{x}: C^{k}\left(M_{j}, G\right) \rightarrow G$ is smooth and $\varepsilon_{x}=\bar{\varepsilon}_{x} \circ \rho_{j}$. We know that $\phi_{j}(v):=\left(L\left(\bar{\varepsilon}_{x}\right)(v)\right)_{x \in M_{j}} \in C^{k}\left(M_{j}, \mathfrak{g}\right)$ for all $v \in L\left(C^{k}\left(M_{j}, G\right)\right)$ and that $\phi_{j}: L\left(C^{k}\left(M_{j}, G\right)\right) \rightarrow C^{k}\left(M_{j}, \mathfrak{g}\right)$ is an isomorphism of topological Lie algebras. For each $v \in L\left(C^{k}(M, G)\right)$, we have

$$
\left(L\left(\varepsilon_{x}\right)(v)\right)_{x \in M_{j}}=\left(L\left(\bar{\varepsilon}_{x}\right)\left(L\left(\rho_{j}\right)(v)\right)\right)_{x \in M_{j}}=\phi_{j}\left(L\left(\rho_{j}\right)(v)\right) \in C^{k}\left(M_{j}, \mathfrak{g}\right)
$$

for $j \in\{1, \ldots, n\}$, whence $\phi(v):=\left(L\left(\varepsilon_{x}\right)(v)\right)_{x \in M} \in C^{k}(M, \mathfrak{g})$. Let us show that the Lie algebra homomorphism $\phi: L\left(C^{k}(M, G)\right) \rightarrow C^{k}(M, \mathfrak{g})$ is a homeomorphism. Lemma 3.7 entails that the map

$$
r=\left(r_{j}\right)_{j=1}^{n}: C^{k}(M, \mathfrak{g}) \rightarrow \prod_{j=1}^{n} C^{k}\left(M_{j}, \mathfrak{g}\right), \quad f \mapsto\left(\left.f\right|_{M_{j}}\right)_{j=1}^{n}
$$

is a homeomorphism. By the preceding, $r \circ \phi=\left(\phi_{1} \times \cdots \times \phi_{n}\right) \circ\left(L\left(\rho_{j}\right)\right)_{j=1}^{n}$ is a homeomorphism, whence so is $\phi$. Thus, the Lie group structure on $C^{k}(M, G)$ is compatible with evaluations. If $\alpha, L=L_{1} \times \cdots \times L_{m}$ and $f: L \rightarrow C^{k}(M, G)$ are as above and $f$ is $C^{\alpha}$, then $f^{\wedge}$ is $C^{\alpha, k}$ by the above argument. If, conversely, $f^{\wedge}$ is $C^{\alpha, k}$, then $\left.f^{\wedge}\right|_{L \times M_{j}}$ is $C^{\alpha, k}$, whence $\left(\left.f^{\wedge}\right|_{L \times M_{j}}\right)^{\vee}=\rho_{j} \circ f$ is $C^{\alpha}$ for all $j \in\{1, \ldots, n\}$. As a consequence, $\rho \circ f$ is $C^{\alpha}$ and thus also $f$. We have shown that the smooth manifold structure on $C^{k}(M, G)$ is precanonical and hence canonical, as compatibility with the compact-open $C^{k}$-topology was already established. 
Another lemma is useful.

Lemma 6.9 Let $N_{1}, \ldots, N_{m}$ and $M_{1}, \ldots, M_{n}$ be locally compact smooth manifolds with rough boundary, $\alpha \in\left(\mathbb{N}_{0} \cup\{\infty\}\right)^{m}, \beta \in\left(\mathbb{N}_{0} \cup\{\infty\}\right)^{m}$, and $G$ be a Lie group. Abbreviate $N:=N_{1} \times \cdots \times N_{m}$ and $M:=M_{1} \times \cdots \times M_{n}$. Assume that $C^{\beta}(M, G)$ has a pre-canonical smooth manifold structure, using which $C^{\alpha}\left(N, C^{\beta}(M, G)\right)$ has a canonical smooth manifold structure. Endow $C^{\alpha, \beta}(N \times M, G)$ with the pre-canonical smooth manifold structure turning

$$
\Phi: C^{\alpha, \beta}(N \times M, G) \rightarrow C^{\alpha}\left(N, C^{\beta}(M, G)\right), \quad f \mapsto f^{\vee}
$$

into a $C^{\infty}$-diffeomorphism. Assume that there exists a family $\left(K_{i}\right)_{i \in I}$ of compact full submanifolds $K_{i}$ of $N$ whose interiors cover $N$, with the following properties:

(a) For each $i \in I$, we have $K_{i}=K_{i, 1} \times \cdots \times K_{i, m}$ with certain compact full submanifolds $K_{i, \ell} \subseteq N_{\ell}$; and

(b) $C^{\beta}\left(M, C^{\alpha}\left(K_{i}, G\right)\right)$ admits a canonical smooth manifold structure for each $i \in I$, using the canonical smooth manifold structure on $C^{\alpha}\left(K_{i}, G\right)$ provided by Theorem 1.1.

Then, the pre-canonical manifold structure on $C^{\alpha, \beta}(N \times M, G)$ is canonical.

Proof Let $\mathcal{O}$ be the topology on $C^{\alpha, \beta}(N \times M, G)$, equipped with its pre-canonical smooth manifold structure. Using Theorem 1.1, for $i \in I$ we endow $C^{\alpha}\left(K_{i}, C^{\beta}(M, G)\right)$ with a canonical smooth manifold structure; the underlying topology is the compact-open $C^{\alpha}$-topology. The given smooth manifold structure on $C^{\alpha}\left(N, C^{\beta}(M, G)\right)$ being canonical, its underlying topology is the compact-open $C^{\alpha}$-topology, which is initial with respect to the restriction maps

$$
\rho_{i}: C^{\alpha}\left(N, C^{\beta}(M, G)\right) \rightarrow C^{\alpha}\left(K_{i}, C^{\beta}(M, G)\right)
$$

for $i \in I$. We have bijections

$$
C^{\alpha}\left(K_{i}, C^{\beta}(M, G)\right) \cong C^{\alpha, \beta}\left(K_{i} \times M, G\right) \cong C^{\beta, \alpha}\left(M \times K_{i}, G\right) \cong C^{\beta}\left(M, C^{\alpha}\left(K_{i}, G\right)\right)
$$

using in turn the exponential law (in the form (1)), a flip in the factors (cf. Lemma 4.12(a)), and again the exponential law. If, step by step, we transport the smooth manifold structure from the left to the right, we obtain a pre-canonical smooth manifold structure in each step (see Lemmas 4.11 (c) and 4.12 (a)). As pre-canonical structures are unique, the pre-canonical structure obtained on $C^{\beta}\left(M, C^{\alpha}\left(K_{i}, G\right)\right)$ must coincide with the canonical structure which exists by hypothesis. Hence, using this canonical structure, the map

$$
\Psi_{i}: C^{\alpha}\left(K_{i}, C^{\beta}(M, G)\right) \rightarrow C^{\beta}\left(M, C^{\alpha}\left(K_{i}, G\right)\right)
$$

determined by $\Psi(f)(y)(x)=f(x)(y)$ is a $C^{\infty}$-diffeomorphism. Let $\mathcal{L}_{k}$ be the set of compact full submanifolds of $M_{k}$ for $k \in\{1, \ldots, n\}$. Write $\mathcal{L}_{1} \times \cdots \times \mathcal{L}_{n}=: J$. If $j \in J$, then $j=\left(L_{j, 1}, \ldots, L_{j, n}\right)$ with certain compact full submanifolds $L_{j, k} \subseteq M_{k}$; we define $L_{j}:=$ $L_{j, 1} \times \cdots \times L_{j, n}$. By Lemma 3.7, the topology on $C^{\beta}\left(M, C^{\alpha}\left(K_{i}, G\right)\right)$ is initial with respect to the restriction maps

$$
r_{i, j}: C^{\beta}\left(M, C^{\alpha}\left(K_{i}, G\right)\right) \rightarrow C^{\beta}\left(L_{j}, C^{\alpha}\left(K_{i}, G\right)\right),
$$

using the compact-open $C^{\alpha}$-topology on the range which underlies the canonical smooth manifold structure given by Theorem 1.1. Let $\Theta_{i, j}$ be the composition of the bijections

$$
C^{\beta}\left(L_{j}, C^{\alpha}\left(K_{i}, G\right)\right) \rightarrow C^{\beta, \alpha}\left(L_{j} \times K_{i}, G\right) \rightarrow C^{\alpha, \beta}\left(K_{i} \times L_{j}, G\right) ;
$$


thus $\Theta_{i, j}(f)(x, y)=f(y)(x)$. As each of the domains and ranges admits a canonical smooth manifold structure (by Theorem 1.1), all of the maps have to be homeomorphisms (see Proposition 4.13 and Lemma 4.12 (b)). Thus, $\Theta_{i, j}$ is a homeomorphism. By transitivity of initial topologies, $\mathcal{O}$ is initial with respect to the mappings

$$
\rho_{i, j}:=\Theta_{i, j} \circ r_{i, j} \circ \Psi_{i} \circ \rho_{i} \circ \Phi \text { for } i \in I \text { and } j \in J,
$$

which are the restriction maps $C^{\alpha, \beta}(N \times M, G) \rightarrow C^{\alpha, \beta}\left(K_{i} \times L_{j}, G\right)$. Also the compactopen $C^{\alpha, \beta}$-topology on $C^{\alpha, \beta}(N \times M, G)$ is initial with respect to the maps $\rho_{i, j}$, and hence coincides with $\mathcal{O}$. The given pre-canonical smooth manifold structure on $C^{\alpha, \beta}(N \times M, G)$ therefore is canonical.

Lemma 6.10 Let $M_{1}, \ldots, M_{n}$ be locally compact, smooth manifold with rough boundary, $M:=M_{1} \times \cdots \times M_{n} \alpha \in\left(\mathbb{N}_{0} \cup\{\infty\}\right)^{n}$, and $G$ be a Lie group. Assume that the group $C^{\alpha}(M, G)$ is endowed with a smooth manifold structure which makes it a Lie group and is compatible with evaluations. Let $\sigma$ be a permutation of $\{1, \ldots, n\}$ and $Q:=M_{\sigma(1)} \times \cdots \times$ $M_{\sigma(n)}$. Consider $\phi_{\sigma}: M \rightarrow Q, x \mapsto x \circ \sigma$. Then, the smooth manifold (and Lie group) structure on the group $C^{\alpha \circ \sigma}(Q, G)$ making the bijective group homomorphism

$$
\left(\phi_{\sigma}\right)^{*}: C^{\alpha \circ \sigma}(Q, G) \rightarrow C^{\alpha}(M, G), f \mapsto f \circ \phi_{\sigma}
$$

$a C^{\infty}$-diffeomorphism is compatible with evaluations.

Proof The map $\psi: C^{\alpha \circ \sigma}(Q, L(G)) \rightarrow C^{\alpha}(M, L(G)), f \mapsto f \circ \phi_{\phi}$ is an isomorphism of topological vector spaces, by Example 4.1 and Lemma 4.12 (b). Write $\bar{\varepsilon}_{y}: C^{\alpha \circ \sigma}(Q, G) \rightarrow G$ for the point evaluation at $y \in Q$ and $\varepsilon_{x}: C^{\alpha}(M, G) \rightarrow G$ for the point evaluation at $x \in M$. For $v \in L\left(C^{\alpha}(M, G)\right)$, let $\phi(v):=\left(L\left(\varepsilon_{x}(v)\right)_{x \in M}\right.$. Then, $\varepsilon_{x} \circ\left(\phi_{\sigma}\right)^{*}=\bar{\varepsilon}_{\phi_{\sigma}(x)}$. As a consequence,

$$
\bar{\phi}(v):=\left(L\left(\bar{\varepsilon}_{y}\right)(v)\right)_{y \in Q}=\left(\psi^{-1} \circ \phi \circ L\left(\left(\phi_{\sigma}\right)^{*}\right)\right)(v) \in C^{\alpha \circ \sigma}(Q, L(G))
$$

for all $v \in L\left(C^{\alpha \circ \sigma}(Q, G)\right)$. Moreover, $\bar{\phi}=\left(\psi^{-1}\right)^{*} \circ \phi \circ L\left(\left(\phi_{\sigma}\right)^{*}\right)$ is an isomorphism of topological vector spaces, being a composition of such.

Proof of Theorem 1.3 and Proposition 6.6. Step 1. We first assume that $M_{j}$ is 1-dimensional with finitely many components for all $j \in\{1, \ldots, n\}$, and prove the assertions by induction on $n$. The case $n=1$ was treated in Lemma 6.8. We may therefore assume that $n \geq 2$ and assume that the conclusions hold for $n-1$ factors. We abbreviate $k:=\alpha_{1}, \beta:=\left(\alpha_{2}, \ldots, \alpha_{n}\right)$, and $L:=M_{2} \times \cdots \times M_{n}$. By the inductive hypothesis, $C^{\beta}(L, G)$ admits a canonical smooth manifold structure which makes it a $C^{r}$-regular Lie group and is compatible with evaluations. By the induction base, $C^{k}\left(M_{1}, C^{\beta}(L, G)\right)$ admits a canonical smooth manifold structure making it a $C^{r}$-regular Lie group. Since $C^{\beta}(L, G)$ is canonical, the group homomorphism

$$
\Phi: C^{k, \beta}\left(M_{1} \times L, G\right) \rightarrow C^{k}\left(M_{1}, C^{\beta}(L, G)\right), \quad f \mapsto f^{\vee}
$$

is a bijection (see (4.13)). We endow

$$
C^{\alpha}(M, G)=C^{k, \beta}\left(M_{1} \times L, G\right)
$$

with the smooth manifold structure turning $\Phi$ into a $C^{\infty}$-diffeomorphism. By Lemma 6.4, this structure is pre-canonical, makes $C^{\alpha}(M, G)$ Lie group, and is compatible with evaluations. The Lie group $C^{\alpha}(M, G)$ is $C^{r}$-regular, as $\Phi$ is an isomorphism of Lie groups. Let $C_{1}, \ldots, C_{\ell}$ be the connected components of $M_{1}$. Let $\mathcal{K}$ be the set of compact, full submanifolds $K$ of $M_{1}$. Then, the interiors $K^{o}$ cover $M_{1}$ (as the interiors of connected, compact full submanifolds 
cover each connected component of $M_{1}$, by the proof of Lemma 6.8). Now $C^{k}(K, G)$ admits a canonical smooth manifold structure making it a $C^{r}$-regular Lie group, by Lemma 6.7. Thus, $C^{\beta}\left(L, C^{k}(K, G)\right)$ admits a canonical smooth manifold structure, by the inductive hypothesis. By Lemma 6.9, the pre-canonical smooth manifold structure on $C^{\alpha}(M, G)$ is canonical.

Step 2 (the general case). Let $M_{1}, \ldots, M_{n}$ be arbitrary. Using Lemma 4.12 (a), we may reorder the factors and assume that there exists an $m \in\{0, \ldots, n\}$ such that $M_{j}$ is compact for all $j \in\{1, \ldots, n\}$ with $j \leq m$, while $M_{j}$ is 1-dimensional with finitely many components for all $j \in\{1, \ldots, n\}$ such that $j>m$. If $m=0$, we have the special case just settled. If $m=n$, then all conclusions hold by Lemma 6.7. We may therefore assume that $1 \leq m<n$. We abbreviate $K:=M_{1} \times \cdots \times M_{m}$ and $N:=M_{m+1} \times \cdots \times M_{n}$. Let $\gamma:=\left(\alpha_{1}, \ldots, \alpha_{m}\right)$ and $\beta:=\left(\alpha_{m+1}, \ldots, \alpha_{n}\right)$. By Step $1, C^{\beta}(N, G)$ admits a canonical smooth manifold structure which makes it a $C^{r}$-regular Lie group and is compatible with evaluations. By Lemma 6.7, $C^{\gamma}\left(K, C^{\beta}(N, G)\right)$ admits a canonical smooth manifold structure which makes it a $C^{r}$-regular Lie group and is compatible with evaluations. We give $C^{\alpha}(M, G)=C^{\gamma, \beta}(K \times N, G)$ the smooth manifold structure making the bijection

$$
\Phi: C^{\gamma, \beta}(K \times N, G) \rightarrow C^{\gamma}\left(K, C^{\beta}(N, G)\right), \quad f \mapsto f^{\vee}
$$

a $C^{\infty}$-diffeomorphism. By Lemma 6.4, this smooth manifold structure is pre-canonical, makes $C^{\alpha}(M, G)$ a Lie group, and is compatible with evaluations. The Lie group $C^{\alpha}(M, G)$ is $C^{r}$-regular as $\Phi$ is an isomorphism of Lie groups. Now $C^{\gamma}(K, G)$ admits a canonical smooth manifold structure, which makes it a $C^{r}$-regular Lie group (Lemma 6.7). By Step 1, $C^{\beta}\left(N, C^{\gamma}(K, G)\right)$ admits a canonical smooth manifold structure. The pre-canonical smooth manifold structure on $C^{\alpha}(M, G)$ is therefore canonical, by Lemma 6.9.

The following result complements Theorem 1.3. Under a restrictive hypothesis, it provides a Lie group structure without recourse to regularity.

Proposition 6.11 Let $M_{1}, \ldots, M_{n}$ be locally compact smooth manifolds with rough boundary, $\alpha \in\left(\mathbb{N}_{0} \cup\{\infty\}\right)^{k}$ and $G$ be a Lie group that is $C^{\infty}$-diffeomorphic to a locally convex space E. Abbreviate $M:=M_{1} \times \cdots \times M_{n}$. Then, $C^{\alpha}(M, G)$ admits a canonical $C^{\infty}$-manifold structure, which is compatible with evaluations. If $G$ is $C^{r}$-regular for some $r \in \mathbb{N}_{0} \cup\{\infty\}$, then also $C^{\alpha}(M, G)$ is $C^{r}$-regular.

Proof By Example 4.1, $H:=C^{\alpha}(M, G)$ admits a canonical smooth manifold structure and this structure makes it a Lie group (see Lemma 6.1). Let $\psi: G \rightarrow E$ be a $C^{\infty}$-diffeomorphism such that $\psi(e)=0$. Abbreviating $\mathfrak{g}:=L(G)$ and $\mathfrak{h}:=L(H)$, the map $\alpha:=\left.d \psi\right|_{\mathfrak{g}}: \mathfrak{g} \rightarrow E$ is an isomorphism of topological vector spaces. Then also, $\phi:=\alpha^{-1} \circ \psi: G \rightarrow E$ is a $C^{\infty}$-diffeomorphism such that $\phi(e)=0$; moreover, $\left.d \phi\right|_{\mathfrak{g}}=\mathrm{id}_{\mathfrak{g}}$. Now

$$
\phi_{*}: C^{\alpha}(M, G) \rightarrow C^{\alpha}(M, \mathfrak{g}), f \mapsto \phi \circ f
$$

is a $C^{\infty}$-diffeomorphism, and thus $\beta:=\left.d\left(\phi_{*}\right)\right|_{\mathfrak{h}}: \mathfrak{h} \rightarrow C^{\alpha}(M, \mathfrak{g})$ is an isomorphism of topological vector spaces. For $x \in M$, let $\varepsilon_{x}: H \rightarrow G$ and $e_{x}: C^{\alpha}(M, \mathfrak{g}) \rightarrow \mathfrak{g}$ be the respective point evaluation at $x$. We show that $\beta(v)=\left(L\left(\varepsilon_{x}\right)(v)\right)_{x \in M}$ for each $v \in \mathfrak{h}$, whence the Lie group structure on $H$ is compatible with evaluations. Regard $v=[\gamma]$ as a geometric tangent vector. As $L\left(\varepsilon_{x}\right)(v) \in \mathfrak{g}$, we have

$$
\begin{aligned}
L\left(\varepsilon_{x}\right)(v) & =d \phi\left(L\left(\varepsilon_{x}\right)(v)\right)=d\left(\phi \circ \varepsilon_{x}\right)(v)=\left.\frac{\mathrm{d}}{\mathrm{d} t}\right|_{t=0}\left(\phi \circ \varepsilon_{x} \circ \gamma\right)(t) \\
& =\left.\frac{\mathrm{d}}{\mathrm{d} t}\right|_{t=0}\left(e_{x} \circ \phi_{*} \circ \gamma\right)(t)=\left.e_{x} \frac{\mathrm{d}}{\mathrm{d} t}\right|_{t=0}\left(\phi_{*} \circ \gamma\right)(t)=d\left(\phi_{*}\right)(v)(x),
\end{aligned}
$$


since $\left(\phi \circ \varepsilon_{x} \circ \gamma\right)(t)=\phi(\gamma(t)(x))=(\phi \circ \gamma(t))(x)=e_{x}\left(\phi_{*}(\gamma(t))\right)=\left(e_{x} \circ \phi_{*} \circ \gamma\right)(t)$ and $e_{x}$ is continuous and linear. For the final assertion, see Lemma 6.5.

\section{Manifolds of maps with finer topologies}

We now turn to manifold structures on $C^{\alpha}(M, N)$ for non-compact $M$, which are modeled on suitable spaces of compactly supported $C^{\alpha}$-functions. Notably, a proof for Theorem 1.4 will be provided. Such manifold structures need not be compatible with the compact-open $C^{\alpha}$-topology, and need not be pre-canonical. But we can essentially reduce their structure to the case of canonical structures for compact domains, using box products of manifolds as a tool. We recall pertinent concepts from [14].

7.1 If $I$ is a non-empty set and $\left(M_{i}\right)_{i \in I}$ a family of $C^{\infty}$-manifolds modeled on locally convex spaces, then the fine box topology $\mathcal{O}_{\mathrm{fb}}$ on the Cartesian product $P:=\prod_{i \in I} M_{i}$ is defined as the final topology with respect to the mappings

$$
\Theta_{\phi}: \bigoplus_{i \in I} V_{i}:=\left(\bigoplus_{i \in I} E_{i}\right) \cap \prod_{i \in I} V_{i} \rightarrow P, \quad\left(x_{i}\right)_{i \in I} \mapsto\left(\phi_{i}^{-1}\left(x_{i}\right)\right)_{i \in I},
$$

for $\phi:=\left(\phi_{i}\right)_{i \in I}$ ranging through the families of charts $\phi_{i}: U_{i} \rightarrow V_{i} \subseteq E_{i}$ of $M_{i}$ such that $0 \in V_{i}$; here $E_{\phi}:=\bigoplus_{i \in I} E_{i}$ is endowed with the locally convex direct sum topology, and the left-hand side $V_{\phi}$ of (9), which is an open subset of $E_{\phi}$, is endowed with the topology induced by $E_{\phi}$. Let $U_{\phi}:=\Theta_{\phi}\left(V_{\phi}\right)$. Thus,

$$
U_{\phi}=\left\{\left(y_{i}\right)_{i \in I} \in \prod_{i \in I} U_{i}: y_{i} \neq \phi_{i}^{-1}(0) \text { for only finitely many } i \in I\right\} .
$$

Note that the projection $\operatorname{pr}_{i}: P \rightarrow M_{i}$ is continuous for each $i \in I$, entailing that the fine box topology is Hausdorff. In fact, using the continuous linear projection $\pi_{i}: E_{\phi} \rightarrow E_{i}$ onto the $i$ th component, we deduce from the continuity of $\operatorname{pr}_{i} \circ \Theta_{\phi}=\left.\phi_{i}^{-1} \circ \pi_{i}\right|_{V_{\phi}}$ for each $\phi$ that $\operatorname{pr}_{i}$ is continuous.

7.2 Let $\phi$ be as before and $\psi$ be an analogous family of charts $\psi_{i}: R_{i} \rightarrow S_{i} \subseteq F_{i}$. If $\phi_{i}^{-1}(0)=\psi_{i}^{-1}(0)$ for all but finitely many $i \in I$, then

$$
\left(\Theta_{\phi}\right)^{-1}\left(U_{\phi} \cap U_{\psi}\right)=\bigoplus_{i \in I} \phi_{i}\left(U_{i} \cap R_{i}\right),
$$

which is an open subset of $\bigoplus_{i \in I} E_{i}$. The transition map

$$
\left(\Theta_{\phi}\right)^{-1} \circ \Theta_{\psi}: \bigoplus_{i \in I} \psi_{i}\left(U_{i} \cap R_{i}\right) \rightarrow \bigoplus_{i \in I} \phi_{i}\left(U_{i} \cap R_{i}\right), \quad\left(x_{i}\right)_{i \in I} \mapsto\left(\left(\phi_{i} \circ \psi_{i}^{-1}\right)\left(x_{i}\right)\right)_{i \in I}
$$

is $C^{\infty}$ (as follows from [11, Proposition 7.1]) and in fact a $C^{\infty}$-diffeomorphism, and hence a homeomorphism, since $\Theta_{\psi}^{-1} \circ \Theta_{\phi}$ is the inverse map. If $\phi_{i}^{-1}(0) \neq \psi_{i}^{-1}(0)$ for infinitely many $i \in I$, then $\left(\Theta_{\phi}\right)^{-1}\left(U_{\phi} \cap U_{\psi}\right)=\varnothing$ and the transition map trivially is a homeomorphism. Using a standard argument, we now deduce that $U_{\phi}=\Theta_{\phi}\left(V_{\phi}\right)$ is open in $\left(P, \mathcal{O}_{\mathrm{fb}}\right)$ for all $\phi$ and $\Theta_{\phi}$ is a homeomorphism onto its image (see, e.g., [15, Exercise A.3.1]). By the preceding, the maps $\Phi_{\phi}:=\left(\left.\Theta_{\phi}\right|^{U_{\phi}}\right)^{-1}: U_{\phi} \rightarrow V_{\phi} \subseteq E_{\phi}$ are smoothly compatible and hence form an atlas for a $C^{\infty}$-manifold structure on $P$. Following [14], we write $P^{\mathrm{fb}}$ for $P$, endowed with the topology $\mathcal{O}_{\mathrm{fb}}$ and the smooth manifold structure just described, and call $P^{\mathrm{fb}}$ the fine box product. 
Some auxiliary results are needed. We use notation as in 5.8 and Theorem 1.4.

Lemma 7.3 Let $M:=M_{1} \times \cdots \times M_{n}$ be a product of locally compact smooth manifolds with rough boundary, $N$ be a smooth manifold, $\alpha \in\left(\mathbb{N}_{0} \cup\{\infty\}\right)^{n}$ and $f \in C^{\alpha}(M, N)$.

(a) If $M_{1}, \ldots, M_{n}$ are compact, then the following bilinear map is continuous:

$$
C^{\alpha}(M, \mathbb{R}) \times \Gamma_{f} \rightarrow \Gamma_{f}, \quad(h, \tau) \mapsto h \tau \text { with }(h \tau)(x)=h(x) \tau(x) .
$$

(b) If $M_{1}, \ldots, M_{n}$ are paracompact, $L \subseteq M$ is a compact subset and $K:=K_{1} \times \cdots \times K_{n}$ with compact full submanifolds $K_{j} \subseteq M_{j}$ for $j \in\{1, \ldots, n\}$, then the linear map $\Gamma_{f, L} \rightarrow \Gamma_{\left.f\right|_{K}},\left.\tau \mapsto \tau\right|_{K}$ is continuous.

(c) If $M_{1}, \ldots, M_{n}$ are paracompact, $K:=K_{1} \times \cdots \times K_{n}$ with compact full submanifolds $K_{j} \subseteq M_{j}$ for $j \in\{1, \ldots, n\}$ and $L \subseteq K$ be compact. Then, $r: \Gamma_{f, L} \rightarrow \Gamma_{\left.f\right|_{K}, L}$, $\left.\tau \mapsto \tau\right|_{K}$ is an isomorphism of topological vector spaces.

Proof (a) The bilinear map is a restriction of the continuous mapping $\mu: C^{\alpha}(M, \mathbb{R}) \times$ $C^{\alpha}(M, T N) \rightarrow C^{\alpha}(M, T N)$ from Lemma 3.12.

(b) The map is a restriction of the restriction map $C^{\alpha}(M, T N) \rightarrow C^{\alpha}(K, T N)$, which is continuous (see Remark 3.6).

(c) For each $x$ in the open subset $M \backslash K$ of $M$, there exist compact full submanifolds $K_{x, j} \subseteq M_{j}$ for $j \in\{1, \ldots, n\}$ such that $K_{x}:=K_{x, 1} \times \cdots \times K_{x, n} \subseteq M \backslash K$ and $x \in K_{x}^{o}$. Lemma 3.7 implies that the compact-open $C^{\alpha}$-topology on $\Gamma_{f, L}$ is initial with respect to the restriction maps $\rho: \Gamma_{f, L} \rightarrow C^{\alpha}(K, T N)$ and $\rho_{x}: \Gamma_{f, L} \rightarrow C^{\alpha}\left(K_{x}, T N\right)$ for $x \in M \backslash K$. As each $\rho_{x}$ is constant (its value is the function $K_{x} \in y \mapsto 0_{f(y)} \in T_{f(y)} N$ ), it can be omitted without affecting the initial topology. The topology on $\Gamma_{f, K}$ is therefore initial with respect to $\rho$, and hence also with respect to the co-restriction $r$ of $\rho$. Thus, $r$ is a topological embedding and hence a homeomorphism, as $r(\tau)=\sigma$ can be achieved for $\sigma \in \Gamma_{\left.f\right|_{K}, L}$ if we define $\tau: M \rightarrow T N$ piecewise via $\tau(x):=\sigma(x)$ if $x \in K$, $\tau(x):=0_{f(x)} \in T_{f(x)} N$ if $x \in M \backslash L$. Being linear, $r$ is an isomorphism of topological vector spaces.

Proof of Theorem 1.4. For $j \in\{1, \ldots, n\}$, let $\left(K_{j, i}\right)_{i \in I_{j}}$ be a locally finite family of compact, full submanifolds $K_{j, i}$ of $M_{j}$ whose interiors cover $M_{j}$. Let $I:=I_{1} \times \cdots \times I_{n}$. Then, the sets $K_{i}:=K_{1, i_{1}} \times \cdots \times K_{n, i_{n}}$ form a locally finite family of compact full submanifolds of $M$ whose interiors cover $M$, for $i=\left(i_{1}, \ldots, i_{n}\right) \in I$. The map

$$
\rho: C^{\alpha}(M, N) \rightarrow \prod_{i \in I} C^{\alpha}\left(K_{i}, N\right), \quad f \mapsto\left(\left.f\right|_{K_{i}}\right)_{i \in I}
$$

is injective with image

$$
\operatorname{im}(\rho)=\left\{\left(f_{i}\right)_{i \in I} \in \prod_{i \in I} C^{\alpha}\left(K_{i}, N\right):(\forall i, j \in I)\left(\forall x \in K_{i} \cap K_{j}\right) f_{i}(x)=f_{j}(x)\right\} .
$$

In fact, the inclusion " $\subseteq$ " is obvious. If $\left(f_{i}\right)_{i \in I}$ is in the set on the right-hand side, then a piecewise definition, $f(x):=f_{i}(x)$ if $x \in K_{i}$, gives a well-defined function $f: M \rightarrow N$ which is $C^{\alpha}$ since $\left.f\right|_{\left(K_{i}\right)^{o}}=\left.f_{i}\right|_{\left(K_{i}\right)^{o}}$ is $C^{\alpha}$ for each $i \in I$. Then, $\rho(f)=\left(f_{i}\right)_{i \in I}$.

For each $i \in I$, endow $C^{\alpha}\left(K_{i}, N\right)$ with the canonical smooth manifold structure, as in Theorem 1.1, modeled on the set $\left\{\Gamma_{f}: f \in C^{\alpha}\left(K_{i}, N\right)\right\}$ of the locally convex spaces $\Gamma_{f}:=$ $\left\{\tau \in C^{\alpha}\left(K_{i}, T N\right): \pi_{T N} \circ \tau=f\right\}$ for $f \in C^{\alpha}\left(K_{i}, N\right)$. Let $\Sigma: T N \supseteq U \rightarrow N$ be a local addition for $N$; as in Sect. 5, write $U^{\prime}:=\left\{\left(\pi_{T N}(v), \Sigma(v)\right): v \in U\right\}$ and $\theta:=$ $\left(\left.\pi_{T N}\right|_{U}, \Sigma\right): U \rightarrow U^{\prime}$. For $f \in C^{\alpha}\left(K_{i}, N\right)$, consider $O_{f}:=\Gamma_{f} \cap C^{\alpha}\left(K_{i}, U\right), O_{f}^{\prime}:=$ 
$\left\{g \in C^{\alpha}\left(K_{i}, N\right):(f, g) \in C^{\alpha}\left(K_{i}, U^{\prime}\right)\right\}$, and $\phi_{f}: O_{f} \rightarrow O_{f}^{\prime}, \tau \mapsto \Sigma \circ \tau$ as in Sect. 5. For $f \in C^{\alpha}(M, N)$, let $\Gamma_{f}$ be the set of all $\tau \in C^{\alpha}(M, T N)$ such that $\pi_{T N} \circ \tau=f$ and

$$
\left\{x \in M: \tau(x) \neq 0_{f(x)} \in T_{f(x)} N\right\}
$$

is relatively compact in $M$. Define $O_{f}:=\Gamma_{f} \cap C^{\alpha}(M, U)$ and let $O_{f}^{\prime}$ be the set of all $g \in C^{\alpha}(M, N)$ such that

$$
(f, g) \in C^{\alpha}\left(M, U^{\prime}\right) \text { and }\left.g\right|_{M \backslash K}=\left.f\right|_{M \backslash K} \text { for some compact subset } K \subseteq M .
$$

Then, $\phi_{f}: O_{f} \rightarrow O_{f}^{\prime}, \tau \mapsto \Sigma \circ \tau$ is a bijection with $\left(\phi_{f}\right)^{-1}(g)=\theta^{-1} \circ(f, g)$. The linear map

$$
s: \Gamma_{f} \rightarrow \bigoplus_{i \in I} \Gamma_{\left.f\right|_{K_{i}}}, \quad \tau \mapsto\left(\left.\tau\right|_{K_{i}}\right)_{i \in I}
$$

is continuous on $\Gamma_{f, L}$ for each compact subset $L \subseteq M$ (see Lemma 7.3(b)) and hence continuous on the locally convex direct limit $\Gamma_{f}$. As above, we see that

$$
\operatorname{im}(s)=\left\{\left(\tau_{i}\right)_{i \in I} \in \bigoplus_{i \in I} \Gamma_{\left.f\right|_{K_{i}}}:(\forall i, j \in I)\left(\forall x \in K_{i} \cap K_{j}\right) \tau_{i}(x)=\tau_{j}(x)\right\},
$$

which is a closed vector subspace of $\bigoplus_{i \in I} \Gamma_{\left.f\right|_{K_{i}}}$. We now show that $s$ is a homeomorphism onto its image. In fact, $s$ admits a continuous linear left inverse. To see this, pick a $C^{\infty}$-partition of unity $\left(h_{i}\right)_{i \in I}$ on $M$ subordinate to $\left(K_{i}^{o}\right)_{i \in I}$; then $L_{i}:=\operatorname{supp}\left(h_{i}\right)$ is a closed subset of $K_{i}$ and thus compact. The multiplication operator $\beta_{i}: \Gamma_{\left.f\right|_{K_{i}}} \rightarrow \Gamma_{\left.f\right|_{K_{i}}, L_{i}}, \tau \mapsto h_{i} \tau$ is continuous linear (by Lemma 7.3(a)). Moreover, the restriction operator $s_{i}: \Gamma_{f, L_{i}} \rightarrow \Gamma_{\left.f\right|_{K_{i}}, L_{i}}$ is an isomorphism of topological vector spaces (Lemma 7.3(c)). Thus, $s_{i}^{-1} \circ \beta_{i}: \Gamma_{\left.f\right|_{K_{i}}} \rightarrow \Gamma_{f, L_{i}} \subseteq$ $\Gamma_{f}$ is a continuous linear map. By the universal property of the locally convex direct sum, also the linear map

$$
\sigma: \bigoplus_{i \in I} \Gamma_{\left.f\right|_{K_{i}}} \rightarrow \Gamma_{f}, \quad\left(\tau_{i}\right)_{i \in I} \mapsto \sum_{i \in I}\left(s_{i}^{-1} \circ \beta_{i}\right)\left(\tau_{i}\right)
$$

is continuous. Hence, $\left.\sigma\right|_{\operatorname{im}(s)}$ is continuous and linear. We easily verify that $\sigma \circ s=\operatorname{id}_{\Gamma_{f}}$. Abbreviate $\phi_{i}:=\left(\phi_{\left.f\right|_{K_{i}}}\right)^{-1}$ and $\phi:=\left(\phi_{i}\right)_{i \in I}$. We now use the $C^{\infty}$-diffeomorphism

$$
\Theta_{\phi}: \bigoplus_{i \in I} O_{\left.f\right|_{K_{i}}} \rightarrow U_{\phi},\left(\tau_{i}\right)_{i \in I} \mapsto\left(\phi_{i}^{-1}\left(\tau_{i}\right)\right)_{i \in I}=\left(\Sigma \circ \tau_{i}\right)_{i \in I}
$$

from 7.1, the inverse of which is the chart

$$
\Phi_{\phi}: U_{\phi} \rightarrow \bigoplus_{i \in I} O_{\left.f\right|_{K_{i}}}, \quad\left(g_{i}\right)_{i \in I} \mapsto\left(\phi_{i}\left(g_{i}\right)\right)_{i \in I}
$$

of $\prod_{i \in I}^{\mathrm{fb}} C^{\alpha}\left(K_{i}, N\right)$ around $\left(\left.f\right|_{K_{i}}\right)_{i \in I}$. For $\left(\tau_{i}\right)_{i \in I} \in \bigoplus_{i \in I} O_{\left.f\right|_{K_{i}}}$, we have

$$
\Theta_{\phi}\left(\left(\tau_{i}\right)_{i \in I}\right) \in \operatorname{im}(\rho) \Leftrightarrow\left(\tau_{i}\right)_{i \in I} \in \operatorname{im}(s) .
$$

In fact, for $i, j \in I$ and $x \in K_{i} \cap K_{j}$ we have $\Sigma\left(\tau_{i}(x)\right)=\Sigma\left(\tau_{j}(x)\right)$ if and only if $\tau_{i}(x)=$ $\tau_{j}(x)$, from which the assertion follows in view of (10) and (11). Thus,

$$
\Phi_{\phi}\left(\operatorname{im}(\rho) \cap U_{\phi}\right)=\operatorname{im}(s) \cap \bigoplus_{i \in I} O_{\left.f\right|_{K_{i}}},
$$


showing that $\operatorname{im}(\rho)$ is a submanifold of $\prod_{i \in I}^{\mathrm{fb}} C^{\alpha}\left(K_{i}, N\right)$. Let

$$
\Psi_{\phi}: \operatorname{im}(\rho) \cap U_{\phi} \rightarrow \operatorname{im}(s) \cap \bigoplus_{i \in I} O_{\left.f\right|_{K_{i}}}, \quad\left(g_{i}\right)_{i \in I} \mapsto \Phi_{\phi}\left(\left(g_{i}\right)_{i \in I}\right)
$$

be the corresponding submanifold chart for $\operatorname{im}(\rho)$. Then,

$$
\rho\left(O_{f}^{\prime}\right)=\operatorname{im}(\rho) \cap U_{\phi} \text { and } s\left(O_{f}\right)=\operatorname{im}(s) \cap \bigoplus_{i \in I} O_{\left.f\right|_{K_{i}}} .
$$

Hence, $\left(\phi_{f}\right)^{-1}=\left.s^{-1} \circ \Psi_{\phi} \circ \rho\right|_{O_{f}^{\prime}}: O_{f}^{\prime} \rightarrow O_{f}$ is a chart for the smooth manifold structure on $C^{\alpha}(M, N)$ modeled on $\mathcal{E}$ (the set of all $\Gamma_{f}$ ) which makes $\rho: C^{\alpha}(M, N) \rightarrow \operatorname{im}(\rho)$ a $C^{\infty}$. diffeomorphism. Note that the smooth manifold structure on $C^{\alpha}(M, N)$ which is modeled on $\mathcal{E}$ and makes $\rho$ a $C^{\infty}$-diffeomorphism is uniquely determined by these properties. Thus, it is independent of the choice of $\Sigma$. On the other hand, the $\left(\phi_{f}\right)^{-1}$ form a $C^{\infty}$-atlas for a given local addition $\Sigma$. As the definition of the $\phi_{f}$ does not involve the cover $\left(K_{i}\right)_{i \in I}$, the smooth manifold structure just constructed is independent of the choice of $\left(K_{i}\right)_{i \in I}$.

Acknowledgements The authors would like to thank the mathematical institute at NTNU Trondheim for its hospitality while conducting the work presented in this article, as well as Nord universitet Levanger.

Data availability Data sharing is not applicable to this article as no datasets were generated or analyzed during the current study.

Open Access This article is licensed under a Creative Commons Attribution 4.0 International License, which permits use, sharing, adaptation, distribution and reproduction in any medium or format, as long as you give appropriate credit to the original author(s) and the source, provide a link to the Creative Commons licence, and indicate if changes were made. The images or other third party material in this article are included in the article's Creative Commons licence, unless indicated otherwise in a credit line to the material. If material is not included in the article's Creative Commons licence and your intended use is not permitted by statutory regulation or exceeds the permitted use, you will need to obtain permission directly from the copyright holder. To view a copy of this licence, visit http://creativecommons.org/licenses/by/4.0/.

\section{A Details for Sects. 2 and 3}

In this "Appendix", we provide proofs for preliminaries in Sects. 2 and 3.

Proof of Lemma 2.8. The right-hand side $(t, y) \mapsto y \cdot \gamma(t)$ of the differential equation $\dot{y}(t)=$ $y(t) \cdot \gamma(t)$ is $C^{k}$, whence its solution $\eta$ will be $C^{k+1}$, if it exists.

To verify existence and uniqueness of $\eta$, we may assume that $I$ is a non-degenerate compact interval with initial point 0 or endpoint 0 , since $I$ is covered by such intervals. Thus, let $I$ be a line segment joining 0 and $\tau \neq 0$. Define $\xi:[0,1] \rightarrow \mathfrak{g}$ via $\xi(t):=\tau \gamma(t \tau)$. By the chain rule, a $C^{1}$-function $\eta: I \rightarrow G$ with $\eta(0)=e$ satisfies $\delta^{\ell} \eta=\gamma$ if and only if $\theta:[0,1] \rightarrow G$, $t \mapsto \eta(t \tau)$ satisfies $\delta^{\ell} \theta=\xi$. The assertion now follows from the case $I=[0,1]$, which holds by $C^{r}$-semiregularity.

Proof for Lemma 2.14. (a) Let $\lambda: Y \rightarrow F$ be the inclusion map, which is continuous linear and thus smooth. If $\left.f\right|^{Y}$ is $C^{\alpha}$, then also $f=\left.\lambda \circ f\right|^{Y}$ is $C^{\alpha}$, by the chain rule [1, Lemma 3.16]. Conversely, assume that $f$ is $C^{\alpha}$ and $f(U) \subseteq Y$. It suffices to deduce that $\left.f\right|^{Y}$ is $C^{\alpha}$ if $\alpha \in\left(\mathbb{N}_{0}\right)^{n}$. The proof is by induction on $|\alpha|$, and establishes in parallel that $d^{\beta}\left(\left.f\right|^{Y}\right)=\left.\left(d^{\beta} f\right)\right|^{Y}$ for all $\beta \leq \alpha$. If $|\alpha|=0$, the conclusion holds since $\left.f\right|^{Y}$ is continuous. If $|\alpha| \geq 1$, let $j \in\{1, \ldots, n\}$ be minimal with $\alpha_{j}>0$. Then, $d^{\beta}\left(\left.f\right|^{Y}\right)$ exists 
for all $\beta \leq \alpha$ such that $\beta_{j} \leq \alpha_{j}-1$, and equals $\left.\left(d^{\beta} f\right)\right|^{Y}$. If $\beta \leq \alpha$ with $\beta_{j}=\alpha_{j}$, let $x \in U^{o}$ and $y_{i} \in E_{i}^{\beta_{i}}$ for $i \in\{j, \ldots, n\}$. Then, all difference quotients needed to define

$$
d^{\beta} f\left(x, 0, \ldots, 0, y_{j}, y_{j+1}, \ldots, y_{n}\right)
$$

are linear combinations of function values of $d^{\beta-e_{j}} f$ and hence in $Y$. Since $Y$ is closed, the limit $d^{\beta} f\left(x, 0, \ldots, 0, y_{j}, y_{j+1}, \ldots, y_{n}\right)$ is in $Y$ as well, and this remains valid for $x \in U$, by density of $U^{o}$ in $U$. Thus, $\left.\left(d^{\beta} f\right)\right|^{Y}$ is a continuous function which extends $d^{\beta}\left(\left.f\right|_{U^{o}} ^{Y}\right)$. We deduce that $\left.f\right|^{Y}$ is $C^{\alpha}$ and $d^{\beta}\left(\left.f\right|^{Y}\right)=\left.\left(d^{\beta} f\right)\right|^{Y}$.

(b) If $f$ is $C^{\alpha}$, then also $\lambda_{a} \circ f$, using that $\lambda_{a}$ is continuous linear and thus smooth. Conversely, assume that $\lambda_{a} \circ f$ is $C^{\alpha}$ for all $a \in A$. Then,

$$
Y:=\left\{\left(x_{a}\right)_{a \in A} \in \prod_{a \in A} F_{a}:(\forall a \leq b) x_{a}=\lambda_{a, b}\left(x_{b}\right)\right\}
$$

is a closed vector subspace of $\prod_{a \in A} F_{a}$ and the map

$$
\lambda: F \rightarrow Y, \quad x \mapsto\left(\lambda_{a}(x)\right)_{a \in A}
$$

is an isomorphism of topological vector spaces. Let $\mathrm{pr}_{a}: Y \rightarrow F_{a}$ be the projection onto the $a$ th component. Since $\operatorname{pr}_{a} \circ \lambda \circ f=\lambda_{a} \circ f$ is $C^{\alpha}$ for all $a \in A$, the map $\lambda \circ f$ is $C^{\alpha}$ to $\prod_{a \in A} F_{a}$ by [1, Lemma 3.8]. By (a), $\lambda \circ f$ is $C^{\alpha}$ also as a map to $Y$. Thus, $f=\lambda^{-1} \circ(\lambda \circ f)$ is $C^{\alpha}$.

Proof of Lemma 2.15. If $f$ is $C^{\alpha}$, then $\rho_{a} \circ f$ is $C^{\alpha}$ for each $a \in A$, the map $\rho_{a}$ being smooth. Assume that, conversely, $\rho_{a} \circ f$ is smooth for each $a \in A$. Write $\psi=\left(\psi_{1}, \psi_{2}\right)$ with $\psi_{1}: M \rightarrow F$ and $\psi_{2}: M \rightarrow N$. Since $\psi_{a}$ is smooth, $\psi_{a} \circ \rho_{a} \circ f=\left(\lambda_{a} \times \operatorname{id}_{N}\right) \circ \psi \circ f$ is $C^{\alpha}$, whence so is its second component $\psi_{2} \circ f$ (see [1, Lemma 3.8]). Also the first component $\lambda_{a} \circ \psi_{1} \circ f$ is $C^{\alpha}$ for each $a \in A$, whence $\psi_{1} \circ f$ is $C^{\alpha}$ by Lemma 2.14(b). Hence, $\psi \circ f$ is $C^{\alpha}$, by [1, Lemma 3.8], and hence so is $f=\psi^{-1} \circ(\psi \circ f)$.

Proof of Lemma 2.17. The proof is by induction on $m:=m_{1}+\cdots+m_{n}$. If $m=n$, there is nothing to show. Assume that $m>n$. After a permutation of $E_{1}, \ldots, E_{n}$, we may assume that $m_{n} \geq 2$ (cf. Lemma 2.13). Let $\left(\beta_{1}, \ldots, \beta_{n-1}\right) \in \prod_{i=1}^{n-1}\left(\mathbb{N}_{0} \cup\{\infty\}\right)^{m_{i}}$, $\beta_{n}=\left(\beta_{n, 1}, \ldots, \beta_{n, m_{n}-1}\right) \in\left(\mathbb{N}_{0} \cup\{\infty\}\right)^{m_{n}-1}$ such that $\left|\beta_{i}\right| \leq \alpha_{i}$ for all $i \in\{1, \ldots, n\}$. Abbreviate $\beta_{n}^{\prime}:=\left(\beta_{n, 1}, \ldots, \beta_{n, m_{n}-2}\right)$. For all $k, \ell \in \mathbb{N}_{0}$ such that $k+\ell \leq \beta_{n, m_{n}-1}$, the map $f$ is $C^{\beta_{1}, \ldots, \beta_{n-1}, \beta_{n}^{\prime}, k, \ell}$. Hence,

$$
f: \prod_{i=1}^{n-1} \prod_{j=1}^{m_{i}} U_{i, j} \times U_{n, 1} \times \cdots \times U_{n, m_{n}-2} \times\left(U_{n, m_{n}-1} \times U_{n, m_{n}}\right) \rightarrow F
$$

is $C^{\beta_{1}, \ldots, \beta_{n}}$, by [1, Lemma 3.12]. By the inductive hypothesis, $f$ is $C^{\alpha}$.

The following lemma fills in the details for 3.3.

Lemma A.1 Let $M_{1}, \ldots, M_{n}$ and $N$ be smooth manifolds with rough boundary, $M:=M_{1} \times$ $\cdots \times M_{n}$ and $f: M \rightarrow N$ be a $C^{\alpha}$-map with $\alpha \in\left(\mathbb{N}_{0} \cup\{\infty\}\right)^{n}$. Then, $f(\bar{x}, \cdot): M_{n} \rightarrow N$ is $C^{\alpha_{n}}$ for each $\bar{x} \in \bar{M}:=M_{1} \times \cdots \times M_{n-1}$ and

$$
h_{k}: M_{1} \times \cdots \times M_{n-1} \times T^{k}\left(M_{n}\right) \rightarrow T^{k} N, \quad(\bar{x}, v) \mapsto T^{k}(f(\bar{x}, \cdot))(v)
$$

is a $C^{\alpha-k e_{n}}$-map for all $k \in \mathbb{N}_{0}$ such that $k \leq \alpha_{n}$. 
Proof We show by induction on $k_{0} \in \mathbb{N}$ that the conclusion holds with $k \leq k_{0}$ for all functions as described in the lemma, for all $\alpha$ with $\alpha_{n} \geq k_{0}$. Using local charts, we may assume that $U_{j}:=M_{j}$ is a locally convex subset of a locally convex space $E_{j}$ for all $j \in\{1, \ldots, n\}$ and $N$ a locally convex subset of a locally convex space $F$; thus $f$ is a map $U:=U_{1} \times \cdots \times U_{n} \rightarrow F$. The case $k_{0}=0$ being trivial as $h_{0}=f$ is $C^{\alpha}$. Let $1 \leq k_{0} \leq \alpha_{n}$ now. Then,

$$
d^{e_{n}} f: U_{1} \times \cdots \times U_{n} \times E_{n} \rightarrow F
$$

is a $C^{\left(\alpha-e_{n}, 0\right)}$-map. Being linear in the final argument, $d^{e_{n}} f$ is $C^{\alpha-e_{n}}$ as a map

$$
U_{1} \times \cdots \times U_{n-1} \times\left(U_{n} \times E_{n}\right) \rightarrow F
$$

of $n$ variables, i.e., as a map on the domain $T^{e_{n}} U=U_{1} \times U_{n-1} \times T U_{n}$ (see [1, Lemma 3.11]). Let $\mathrm{pr}_{1}: T U_{n}=U_{n} \times E_{n} \rightarrow U_{n}$ be the projection onto the first component. Then, $g:=$ $f \circ \operatorname{id}_{U_{1}} \times \cdots \times \operatorname{id}_{U_{n-1}} \times \mathrm{pr}_{1}: U_{1} \times \cdots \times U_{n-1} \times T U_{n} \rightarrow F$ is $C^{\alpha}$ by the chain rule [1, Lemma 3.16], and hence $C^{\alpha-e_{n}}$. Thus, $h_{1}=\left(g, d^{e_{n}} f\right)$ is $C^{\alpha-e_{n}}$, by [1, Lemma 3.8]. By the inductive hypothesis, the maps

$$
U_{1} \times \cdots \times U_{n-1} \times T^{j}\left(T U_{n}\right) \rightarrow T^{j}(T F), \quad(\bar{x}, v) \mapsto T^{j}\left(h_{1}(x, \cdot)\right)(v)
$$

are $C^{\alpha-e_{n}-j e_{n}}$ for all $j \in\left\{0, \ldots, k_{0}-1\right\}$. It only remains to observe that this map equals $h_{j+1}$.

Proof of Lemma 3.5. (a) For $\beta \in \mathbb{N}_{0}^{n}$ with $\beta \leq \alpha$, consider the maps

$$
T^{\beta}: C^{\alpha}(M, N) \rightarrow C\left(T^{\beta} M, T^{|\beta|} N\right), \quad f \mapsto T^{\beta} f
$$

and $\tau_{\beta}: C^{\alpha}(M, L) \rightarrow C\left(T^{\beta} N, T^{|\beta|} L\right), f \mapsto T^{\beta} f$. Going through the recursive construction of $T^{\beta}(g \circ f)$ in 3.3 for $f \in C^{\alpha}(M, N)$ and making repeated use of the functoriality of $T$, we see that

$$
T^{\beta}(g \circ f)=T^{|\beta|} g \circ T^{\beta} f .
$$

Thus, $\tau_{\beta} \circ C^{\alpha}(M, g)=C\left(T^{\beta} M, T^{|\beta|} g\right) \circ T^{\beta}$, which is a continuous map by [15, Lemma A.6.3]. The topology on $C^{\beta}(M, L)$ being initial with respect to the maps $\tau_{\beta}$, we deduce that $C^{\alpha}(M, g)$ is continuous.

(b) For $\beta \in \mathbb{N}_{0}^{n}$ with $\beta \leq \alpha$, consider the maps $T^{\beta}: C^{\alpha}(M, N) \rightarrow C\left(T^{\beta} M, T^{|\beta|} N\right)$, $f \mapsto T^{\beta} f$ and $\tau_{\beta}: C^{\alpha}(L, N) \rightarrow C\left(T^{\beta} L, T^{|\beta|} N\right), f \mapsto T^{\beta} f$. Going through the recursive construction of $T^{\beta}(f \circ g)$ in 3.3 for $f \in C^{\alpha}(M, N)$ and making repeated use of the functoriality of $T$, we see that

$$
T^{\beta}(f \circ g)=\left(T^{\beta} f\right) \circ h_{\beta}
$$

with $h_{\beta}:=T^{\beta_{1}} g_{1} \times \cdots \times T^{\beta_{n}} g_{n}$. Thus, $\tau_{\beta} \circ C^{\alpha}(g, N)=C\left(h_{\beta}, T^{|\beta|} N\right) \circ T^{\beta}$, which is a continuous map by [15, Lemma A.6.9]. The topology on $C^{\alpha}(L, N)$ being initial with respect to the maps $\tau_{\beta}$, we deduce that $C^{\alpha}(g, N)$ is continuous.

Proof of Lemma 3.7. By definition, the compact-open $C^{\alpha}$-topology $\mathcal{O}$ on $C^{\alpha}(M, N)$ is initial with respect to the maps $\tau_{\beta}: C^{\alpha}(M, N) \rightarrow C\left(T^{\beta} M, T^{|\beta|} N\right), f \mapsto T^{\beta} f$ for $\beta \in\left(\mathbb{N}_{0}\right)^{n}$ such that $\beta \leq \alpha$. As the interiors $\left(T^{\beta} K_{i}\right)^{o}$ cover $T^{\beta} M$, the compact-open topology on $C\left(T^{\beta} M, T^{|\beta|} N\right)$ is initial with respect to the restriction maps $\rho_{\beta, i}: C\left(T^{\beta} M, T^{|\beta|} N\right) \rightarrow$ $C\left(T^{\beta} K_{i}, T^{|\beta|} N\right)$, by [15, Lemma A.6.11]. By transitivity of initial topologies, $\mathcal{O}$ is initial with respect to the mappings $\rho_{\beta, i} \circ \tau_{\beta}$. Let $\rho_{i}: C^{\alpha}(M, N) \rightarrow C^{\alpha}\left(K_{i}, N\right)$ the restriction map. 
The compact-open $C^{\alpha}$-topology on $C^{\alpha}\left(K_{i}, N\right)$ being initial with respect to the mappings $\tau_{\beta, i}: C^{\alpha}\left(K_{i}, N\right) \rightarrow C\left(T^{\beta} K_{i}, T^{|\beta|} N\right), f \mapsto T^{\beta} f$, we deduce from

$$
\rho_{\beta, i} \circ \tau_{\beta}=\tau_{\beta, i} \circ \rho
$$

that $\mathcal{O}$ is initial with respect to the maps $\rho_{i}$.

Proof of Lemma 3.8. The case $n=1$ is well known. The general case follows as $T^{\beta} S=$ $T^{\beta_{1}} S_{1} \times \cdots \times T^{\beta_{n}} S_{n}$ and $T^{\beta} M=T^{\beta_{1}} M_{1} \times \cdots \times T^{\beta_{n}} M_{n}$.

Proof of Lemma 3.9. The inclusion map $\lambda: S \rightarrow N$ is smooth. By Lemma 3.8, the inclusion map $T^{|\beta|} \lambda: T^{|\beta|} S \rightarrow T^{|\beta|} N$ is a topological embedding, for each $\beta \in\left(\mathbb{N}_{0}\right)^{n}$ such that $\beta \leq \alpha$. Thus, $\left(T^{|\beta|} \lambda\right)_{*}: C\left(T^{\beta} M, T^{|\beta|} S\right) \rightarrow C\left(T^{\beta} M, T^{|\beta|} N\right)$ is a topological embedding for the compact-open topologies (see, e.g., [15, Lemma A.6.5]). The compact-open $C^{\alpha}$-topology $\mathcal{O}$ on $C^{\alpha}(M, S)$, which is initial with respect to the maps $\tau_{\beta, S}: C^{\alpha}(M, S) \rightarrow C\left(T^{\beta} M, T^{|\beta|} S\right)$, $f \mapsto T^{\beta} f$ is therefore also initial with respect to the mappings $\left(T^{|\beta|} \lambda\right)_{*} \circ \tau_{\beta, S}$. The compactopen $C^{\alpha}$-topology on $C^{\alpha}(M, N)$ is initial with respect to the maps $\tau_{\beta, N}: C^{\alpha}(M, N) \rightarrow$ $C\left(T^{\beta} M, T^{|\beta|} N\right), f \mapsto T^{\beta} f$. As $\left(T^{|\beta|} \lambda\right)_{*} \circ \tau_{\beta, S}=\tau_{\beta, N} \circ \lambda_{*}$, we see that the topology $\mathcal{O}$ is initial with respect to the inclusion map $\lambda_{*}: C^{\alpha}(M, S) \rightarrow C^{\alpha}(M, N)$. Thus, $\mathcal{O}$ is the induced topology.

Proof of Lemma 3.10. For each $k \in \mathbb{N}_{0}, T^{k} F=F^{2^{k}}$ is a locally convex space. For each $\beta \in\left(\mathbb{N}_{0}\right)^{n}$ such that $\beta \leq \alpha$, the map

$$
T^{\beta}: C^{\alpha}(M, F) \rightarrow C\left(T^{\beta} M, T^{|\beta|} F\right), \quad f \mapsto T^{\beta} f
$$

is linear. In fact, $T^{k}: C^{k}(N, F) \rightarrow C\left(T^{k} N, T^{k} F\right)$ is linear for each smooth manifold $N$ with rough boundary [15, proof of Proposition 4.1.11] and $k \in \mathbb{N}_{0}$, establishing linearity if $n=1$. If $n \geq 2$, the preceding entails that $T^{\left(0, \ldots, 0, \beta_{n}\right)} f(v)=T^{\beta_{n}}\left(f\left(x_{1}, \ldots, x_{n-1}, \cdot\right)\right)\left(v_{n}\right)$ is linear in $f$ for all $x_{j} \in M_{j}$ for $j \in\{1, \ldots, n-1\}$ and $v_{n} \in T^{\beta_{n}} M_{n}$, showing that $T^{\left(0, \ldots, 0, \beta_{n}\right)} f$ is linear in $f$. Likewise, $g$ and $T^{\left(0, \ldots, 0, \beta_{k-1}, \ldots, \beta_{n}\right)} f$ is linear in $f$ in the recursive construction in 3.3, which gives the assertion for $n \geq 2$. Thus,

$$
C^{\alpha}(M, F) \rightarrow \prod_{\beta \leq \alpha} C\left(T^{\beta} M, T^{|\beta|} F\right), f \mapsto\left(T^{\beta} f\right)_{\beta \leq \alpha}
$$

is a linear map. It is a homeomorphism onto its image, which is a locally convex space. Hence also, $C^{\alpha}(M, F)$ is a locally convex space.

Proof of Lemma 3.11. (a) For each $k \in \mathbb{N}_{0}$, the topology on $T^{k} F=F^{2^{k}}$ is initial with respect to the linear maps $T^{k} \lambda_{i}=\lambda_{i}^{2^{k}}: F^{2^{k}} \rightarrow F_{i}^{2^{k}}$. For each $\beta \in \mathbb{N}_{0}^{n}$ with $\beta \leq \alpha$, the compact-open topology on $C\left(T^{\beta} M, T^{|\beta|} F\right)$ is therefore initial with respect to the mappings

$$
C\left(T^{\beta} M, T^{|\beta|} \lambda_{i}\right): C\left(T^{\beta} M, T^{|\beta|} F\right) \rightarrow C\left(T^{\beta} M, T^{|\beta|} F_{i}\right)
$$

for $i \in I$, see [15, Lemma A.6.4]. Thus, the compact-open $C^{\alpha}$-topology $\mathcal{O}$ on $C^{\alpha}(M, F)$ is initial with respect to the maps $C\left(T^{\beta} M, T^{|\beta|} \lambda_{i}\right) \circ T^{\beta}$ with $T^{\beta}: C^{\alpha}(M, F) \rightarrow$ $C\left(T^{\beta} M, T^{|\beta|} F\right)$. As $T^{\beta}\left(\lambda_{i} \circ f\right)=\left(T^{|\beta|} \lambda_{i}\right) \circ\left(T^{\beta} f\right)$, writing $\tau_{i, \beta}(g):=T^{\beta} g$ for $g \in C^{\alpha}\left(M, F_{i}\right)$ we have

$$
C\left(T^{\beta} M, T^{|\beta|} \lambda_{i}\right) \circ T^{\beta}=\tau_{i, \beta} \circ C^{\alpha}\left(M, \lambda_{i}\right) .
$$

The topology on $C^{\alpha}\left(M, F_{i}\right)$ being initial with respect to the mappings $\tau_{i, \beta}: C^{\alpha}\left(M, F_{i}\right) \rightarrow$ $C\left(T^{\beta} M, T^{|\beta|} F_{i}\right)$ for $\beta \leq \alpha$, we deduce that $\mathcal{O}$ is initial with respect to the mappings $C^{\alpha}\left(M, \lambda_{i}\right)=\left(\lambda_{i}\right)_{*}$. 
(b) By [1, Lemma 3.8], the linear map $\Theta$ is a bijection. The topology on $F$ being initial with respect to the maps $\mathrm{pr}_{i}$, (a) shows that the topology on $C^{\alpha}(M, F)$ is initial with respect to the maps $\left(\mathrm{pr}_{i}\right)_{*}$ and hence makes $\Theta$ a topological embedding. Hence, $\Theta$ is a homeomorphism, being bijective.

(c) By [1, Lemma 3.8], $\Psi$ is a bijection. By Lemma 3.5, $\Psi$ is continuous. To see that $\Psi^{-1}$ is continuous, we prove its continuity at a given element $\left(f_{1}, f_{2}\right)$ in $C^{\alpha}\left(M, N_{1}\right) \times$ $C^{\alpha}\left(M, N_{2}\right)$. For $x \in M$, pick a chart $\phi_{x, i}: U_{x, i} \rightarrow V_{x, i} \subseteq E_{x, i}$ of $N_{i}$ around $f_{i}(x)$, for $i \in\{1,2\}$. There exist compact full submanifolds $K_{x, j}$ of $M_{j}$ for $j \in\{1, \ldots, n\}$ such that $K_{x}:=K_{x, 1} \times \cdots \times K_{x, n} \subseteq\left(f_{1}, f_{2}\right)^{-1}\left(U_{x, 1} \times U_{x, 2}\right)$ and $x \in K_{x}^{o}$. By Lemma 3.7, the topology on $C^{\alpha}\left(M, N_{1} \times N_{2}\right)$ is initial with respect to the restriction maps

$$
\rho_{x}: C^{\alpha}\left(M, N_{1} \times N_{2}\right) \rightarrow C^{\alpha}\left(K_{x}, N_{1} \times N_{2}\right) .
$$

It thus suffices to show that $\rho_{x} \circ \Psi^{-1}$ is continuous at $\left(f_{1}, f_{2}\right)$ for all $x \in M$. Now $\rho_{x} \circ$ $\Psi^{-1}=\Psi_{x}^{-1} \circ\left(\rho_{x, 1} \times \rho_{x, 2}\right)$ using the continuous restriction maps $\rho_{x, i}: C^{\alpha}\left(M, N_{i}\right) \rightarrow$ $C^{\alpha}\left(K_{x}, N_{i}\right)$ for $i \in\{1,2\}$ and the map

$$
\Psi_{x}: C^{\alpha}\left(K_{x}, N_{1} \times N_{2}\right) \rightarrow C^{\alpha}\left(K_{x}, N_{1}\right) \times C^{\alpha}\left(K_{x}, N_{2}\right)
$$

taking a function to its pair of components. Thus, it suffices to show that $\Psi_{x}^{-1}$ is continuous at $\left(\left.f_{1}\right|_{K_{x}},\left.f_{2}\right|_{K_{x}}\right)$. Now $\left.f_{i}\right|_{K_{x}}$ is contained in the open subset $C^{\alpha}\left(K_{x}, U_{x, i}\right)$ of $C^{\alpha}\left(K_{x}, N_{i}\right)$, on which the latter induces the compact-open $C^{\alpha}$-topology, by Lemma 3.9. The map $\Psi^{-1}$ takes this set onto $C^{\alpha}\left(M, U_{x, 1} \times U_{x, 2}\right)$, on which $C^{\alpha}\left(M, N_{1} \times N_{2}\right)$ induces the compact-open $C^{\alpha}$-topology. It thus suffices to show that $\Psi_{x}^{-1}$ is continuous at $\left(\left.f_{1}\right|_{K_{x}},\left.f_{2}\right|_{K_{x}}\right)$ as a map

$$
C^{\alpha}\left(K_{x}, U_{x, 1}\right) \times C^{\alpha}\left(K_{x}, U_{x, 2}\right) \rightarrow C^{\alpha}\left(K_{x}, U_{x, 1} \times U_{x, 2}\right) .
$$

Now $\left(\phi_{x, j}\right)_{*}: C^{\alpha}\left(K_{x}, U_{x, j}\right) \rightarrow C^{\alpha}\left(K_{x}, V_{x, i}\right)$ is a homeomorphism for $i \in\{1,2\}$ and also $\left(\phi_{x, 1} \times \phi_{x, 2}\right)_{*}: C^{\alpha}\left(K_{x}, U_{x, 1} \times U_{x, 2}\right) \rightarrow C^{\alpha}\left(K_{x}, V_{x, 1} \times V_{x, 2}\right)$ is a homeomorphism, by Lemma 3.5. It thus suffices to show that the mapping $\left(\phi_{x, 1} \times \phi_{x, 2}\right)_{*} \circ \Psi_{x}^{-1} \circ\left(\left(\phi_{x, 1}\right)_{*} \times\right.$ $\left.\left(\phi_{x, 2}\right)_{*}\right)^{-1}$ :

$$
C^{\alpha}\left(K_{x}, V_{x, 1}\right) \times C^{\alpha}\left(K_{x}, V_{x, 2}\right) \rightarrow C^{\alpha}\left(K_{x}, V_{x, 1} \times V_{x, 2}\right)
$$

is continuous. But this mapping is a restriction of the homeomorphism $C^{\alpha}\left(K_{x}, E_{x, 1}\right) \times$ $C^{\alpha}\left(K_{x}, E_{x, 2}\right) \rightarrow C^{\alpha}\left(K_{x}, E_{x, 1} \times E_{x, 2}\right)$ discussed in (b).

Proof of Lemma 3.12. The scalar multiplication $\sigma: \mathbb{R} \times T N \rightarrow T N$ being smooth, the map $\sigma_{*}: C^{\alpha}(M, \mathbb{R} \times T N) \rightarrow C^{\alpha}(M, T N), h \mapsto \sigma \circ h$ is continuous (see Lemma 3.5). Hence, $\mu=\sigma_{*} \circ \Psi^{-1}$ is continuous, using the homeomorphism $\Psi: C^{\alpha}(M, \mathbb{R} \times T N) \rightarrow C^{\alpha}(M, \mathbb{R}) \times$ $C^{\alpha}(M, T N)$ from Lemma 3.11 .

Proof of Lemma 3.13. Let $\left(U_{i}\right)_{i \in I}$ be the family of pairwise distinct connected components of $N$ and $\left(V_{j}\right)_{j \in J}$ be the family of components of $M$. Then,

$$
r: C^{\beta}(M, E) \rightarrow \prod_{j \in J} C^{\beta}\left(V_{j}, E\right), \quad f \mapsto\left(\left.f\right|_{V_{j}}\right)_{j \in J}
$$

is a bijective linear map; by Lemma 3.7, it is a homeomorphism. Likewise,

$$
\rho: C^{\alpha, \beta}(N \times M, E) \rightarrow \prod_{(i, j) \in I \times J} C^{\alpha, \beta}\left(U_{i} \times V_{j}, E\right), \quad f \mapsto\left(\left.f\right|_{U_{i} \times V_{j}}\right)_{(i, j) \in I \times J}
$$


and $R: C^{\alpha}\left(N, C^{\beta}(M, E)\right) \rightarrow \prod_{i \in I} C^{\alpha}\left(U_{i}, C^{\beta}(M, E)\right), f \mapsto\left(\left.f\right|_{U_{i}}\right)_{i \in I}$ are isomorphisms of topological vector spaces. By Lemma 3.5, the mapping $C^{\alpha}\left(U_{i}, r\right): C^{\alpha}\left(U_{i}, C^{\beta}(M, E)\right) \rightarrow$ $C^{\alpha}\left(U_{i}, \prod_{j \in J} C^{\beta}\left(V_{j}, E\right)\right)$ is an isomorphism of topological vector spaces and so is the map

$$
\Theta_{i}: C^{\alpha}\left(U_{i}, \prod_{j \in J} C^{\beta}\left(V_{j}, E\right)\right) \rightarrow \prod_{j \in J} C^{\alpha}\left(U_{i}, C^{\beta}\left(V_{j}, E\right)\right)
$$

taking a map to its family of components (see Lemma 3.11 (b)). Hence,

$$
\Xi:=\prod_{i \in I} \Theta_{i} \circ \prod_{i \in I} C^{\alpha}\left(U_{i}, r\right) \circ R: C^{\alpha}\left(N, C^{\beta}(M, E)\right) \rightarrow \prod_{(i, j) \in I \times J} C^{\alpha}\left(U_{i}, C^{\beta}\left(V_{j}, E\right)\right)
$$

is an isomorphism of topological vector spaces. By [1, Theorem B], the map $\Phi_{i, j}: C^{\alpha, \beta}\left(U_{i} \times\right.$ $\left.\left.V_{j}, E\right)\right) \rightarrow C^{\alpha}\left(U_{i}, C^{\beta}\left(V_{j}, E\right)\right), f \mapsto f^{\vee}$ is linear and a topological embedding, whence so is

$$
\Psi:=\prod_{(i, j) \in I \times J} \Phi_{i, j}: \prod_{(i, j) \in I \times J} C^{\alpha, \beta}\left(U_{i} \times V_{j}, E\right) \rightarrow \prod_{(i, j) \in I \times J} C^{\alpha}\left(U_{i}, C^{\beta}\left(V_{j}, E\right)\right) .
$$

Evaluating at $x \in N$ and then in $y \in M$ (say $x \in U_{i}$ and $y \in V_{j}$ ), we verify that

$$
f^{\vee}=\left(\Xi^{-1} \circ \Psi \circ \rho\right)(f)
$$

for all $f \in C^{\alpha, \beta}(N \times M, E)$, whence $f^{\vee} \in C^{\alpha}\left(N, C^{\beta}(M, E)\right)$ and $\Phi$ makes sense as a map to the latter space. We have a commutative diagram

$$
\begin{array}{cc}
C^{\alpha, \beta}(N \times M, E) & \stackrel{\Phi}{\longrightarrow} C^{\alpha}\left(N, C^{\beta}(M, E)\right) \\
\rho \downarrow & \downarrow \Xi \\
\prod_{i, j} C^{\alpha, \beta}\left(U_{i} \times V_{j}, E\right) \stackrel{\Psi}{\longrightarrow} \prod_{i, j} C^{\alpha}\left(U_{i}, C^{\beta}\left(V_{j}, E\right)\right)
\end{array}
$$

where the vertical arrows are homeomorphisms and $\Psi$ is a topological embedding. Hence, $\Phi$ is a topological embedding. If $M$ is locally compact, then so are the $V_{j}$, whence each of the maps $\Phi_{i, j}$ is a homeomorphism by [1, Theorem 4.4] and hence also $\Psi$. Then also, $\Phi=\Xi^{-1} \circ \Psi \circ \rho$ is a homeomorphism.

\section{References}

1. Alzaareer, H.: Differential calculus on multiple products. Indag. Math. 30, 1036-1060 (2019)

2. Alzaareer, H.: Lie groups of $C^{k}$-maps on non-compact manifolds and the fundamental theorem for Lie group-valued mappings. J. Group Theory 24, 1099-1134 (2021)

3. Alzaareer, H., Schmeding, A.: Differentiable mappings on products with different degrees of differentiability in the two factors. Expo. Math. 33, 184-222 (2015)

4. Amiri, H., Glöckner, H., Schmeding, A.: Lie groupoids of mappings taking values in a Lie groupoid. Arch. Math. (Brno) 56, 307-356 (2020)

5. Bastiani, A.: Applications différentiables et variétés différentiables de dimension infinie. J. Anal. Math. 13, 1-114 (1964)

6. Bertram, W., Glöckner, H., Neeb, K.-H.: Differential calculus over general base fields and rings. Expo. Math. 22, 213-282 (2004)

7. Cerf, J.: Topologie de certains éspaces de plongements. Bull. Soc. Math. Fr. 89, 227-380 (1961)

8. Douady, A.: Variétés à bord anguleux et voisinages tubulaires. In: Séminaire Henri Cartan, Exp. 1 $(1961 / 1962)$

9. Eells, J.: On the geometry of function spaces. In: Sympos. internac. Topología algebráica, pp. 303-308 (1958) 
10. Glöckner, H.: Infinite-dimensional Lie groups without completeness restrictions. In: Strasburger, A., et al. (eds.) Geometry and Analysis on Finite- and Infinite-Dimensional Lie Groups, vol. 55, pp. 43-59. Banach Center Publications, Warsaw (2002)

11. Glöckner, H.: Lie groups of measurable mappings. Can. J. Math. 55, 969-999 (2003)

12. Glöckner, H.: Regularity properties of infinite-dimensional Lie groups and semiregularity. Preprint arXiv: 1208.0715

13. Glöckner, H.: Smoothing operators for vector-valued functions and extension operators. Preprint arXiv:2006.00254

14. Glöckner, H.: Manifolds of mappings on rough manifolds and manifold structures on box products, in preparation

15. Glöckner, H., Neeb, K.-H.: Infinite-Dimensional Lie Groups, book in preparation

16. Hamilton, R.S.: The inverse function theorem of Nash and Moser. Bull. Am. Math. Soc. 7, 65-222 (1982)

17. Hanusch, M.: A $C^{k}$-Seeley-extension-theorem for Bastiani’s differential calculus. Can. J. Math. https:// doi.org/10.4153/S0008414X21000596

18. Ingrisch, S.: Gemischte Differenzierbarkeit von Fixpunkten und impliziten Funktionen. Master's thesis, University of Paderborn (2019) (advisor: H. Glöckner)

19. Keller, H.H.: Differential Calculus in Locally Convex Spaces. Springer, Berlin (1974)

20. Kriegl, A., Michor, P.W.: The Convenient Setting of Global Analysis. AMS, Providence (1997)

21. Michor, P.W.: Manifolds of Differentiable Mappings. Shiva, Orpington (1980)

22. Milnor, J.: Remarks on infinite-dimensional Lie groups. In: DeWitt, B.S., Stora, R. (eds.) Relativité, groupes et topologie II, pp. 1007-1057. North-Holland, Amsterdam (1984)

23. Neeb, K.-H.: Towards a Lie theory of locally convex groups. Jpn. J. Math. 1, 291-468 (2006)

24. Neeb, K.-H., Wagemann, F.: Lie group structures on groups of smooth and holomorphic maps on noncompact manifolds. Geom. Dedic. 134, 17-60 (2008)

25. Wittmann, J.: The Banach manifold $C^{k}(M, N)$. Differ. Geom. Appl. 63, 166-185 (2019)

Publisher's Note Springer Nature remains neutral with regard to jurisdictional claims in published maps and institutional affiliations. 